NASA Technical Memorandum 84529

\title{
Simulation of Time-Varying Ascent Loads on Arrays of Shuttle Tiles in a Large Transonic Tunnel
}

Percy J. Bobbitt, C. L. W. Edwards, and Richard W. Barnwell

NOVEMBER 1982 

NASA Technical Memorandum 84529

\section{Simulation of Time-Varying Ascent Loads on Arrays of Shuttle Tiles in a Large Transonic Tunnel}

Percy J. Bobbitt, C. L. W. Edwards, and Richard W. Barnwell

Langley Research Center

Hampton, Virginia

\section{N/Sก}

National Aeronautics

and Space Administration

Scientific and Technical Information Branch 
Use of trade names or names of manufacturers in this report does not constitute an official endorsement of such products or manufacturers, either expressed or implied, by the National Aeronautics and Space Administration. 


\section{SUMMARY}

A large variety of tests were conducted to determine the strength, fatigue, and thermal characteristics of the thermal-protection system of the shuttle orbitex. The present paper describes first-flight-critical tests, conducted in the Langley 8-Foot Transonic Pressure Tunnel (8-ft TPT), which simulated the time histories of Shuttle ascent loads on tile arrays bonded to structures which accurately duplicated those of the shuttle. The time-varying free-stream conditions were provided by controlling the deflection-angle history of diffuser spoiler flaps in an automated way. Time histories of the critical-load parameters imposed on the tile arrays in the tunnel are compared with those expected in flight. In addition, the effect of repeated load pulses on the smoothness of the surface and condition of the tiles is discussed briefly.

\section{INTRODUCTION}

A large variety of tests were conducted to determine the strength, fatigue, and thermal characteristics of the thermal-protection system of the Shuttle orbiter. Included were tests of the individual components, single-tile systems, and tile arrays. Materials, vibroacoustic, aerodynamic load, and aerodynamic heating tests were conducted with models of varying size and complexity in the laboratory, in flight, and in many types of wind tunnels. The present paper describes several tests which were a part of this large program and which involved the simulation in the Langley 8-Foot Transonic Pressure Tunnel (8-ft TPT) of the time histories of Shuttle ascent loads on tiles bonded to realistic structures. This type of test had never before been attempted in a wind tunnel of this size.

The primary objectives of these tests were to verify that the tiles would remain attached to the flight structures under the simulated flight conditions, to compare measured and predicted tile and strain-isolation-pad (SIP) loads and responses, and to determine tile roughness characteristics after single- and repeated-ascent missions. The process for determining the aerodynamic environments on the tile arrays (called panels) selected for testing is described subsequently. This process required the use of previously acquired pressure data on the ascent configuration, as well as data from transonic and supersonic wind-tunnel tests of subscale bipods carried out as a separate part of this program. Precalibration and calibration panels used to obtain detailed loads data, to calibrate the instrumentation, and to ascertain and modify the actual pressure environment in the tunnel are also discussed. Finally, the methodology used to provide the required time-varying free-stream and local conditions is described.

\section{SYMBOLS}

$$
\begin{array}{ll}
C_{p} & =\frac{p-p_{\infty}}{q_{\infty}} \\
D & \text { diameter of bipod legs, in. } \\
L & \text { length of Shuttle orbiter, in. }
\end{array}
$$


M

$\mathrm{p}$

PSCALE nondimensional quantity used in correlating pressure data ahead of bipod

q

t

V

$\mathrm{X}$

$\mathrm{x}_{\text {SCALE }}$

$\mathrm{x}$

$x^{\prime}$

y

$\delta_{\mathbf{F}}$

$\rho$

$\Delta_{\mathrm{SHK}}$

$\Delta \mathrm{p}_{\infty}$

$\Delta_{\text {SHOCK }}$

Mach number

static pressure, psi dynamic pressure, $1 / 2 \rho v^{2}$, psf

time, sec

velocity, ft/sec

dimensional distance measured forward from face of bipod, in.

nondimensional quantity used in correlating pressure data ahead of hipod or distance measured rearward along false floor from leading edge, in.

distance measured forward from face of bipod, in.

lateral distance measured from centerline of Shuttle, in.

diffuser-flap deflection anqle, deq

density, slugs/ft ${ }^{3}$ leg, in. distance measured rearward along centerline of Shuttle from nose of shuttle,

shock detachment distance measured at intersection of base flange with bipod

Subscripts:

t stagnation conditions

LOCAL quantity measured in separated-flow region ahead of bipod

ONSET quantity measured immediately ahead of separated-flow region ahead of bipod

PLATFAU value of pressure in plateau region just downstream of flow separation point ahead of bipod

2

value at a single point (for panel 20A, 52 in. from leading edge of panel;

for panel 20C, 4 bipod diameters ahead of face of bipod (12 in. for a half-scale bipod))

$\infty$

free-stream conditions

Abbreviations:

CLOT combined loads orbiter tests 
DFI diagnostic flight instrumentation

IML inner mold line

OFPL overall fluctuating pressure level, $\mathrm{dB}$

OML outer mold line

PCF pounds per cubic foot

rms root mean square

SIP strain isolation pad

STS-1 first flight of space transportation system

TPS thermal-protection system

UPWT Langley Unitary Plan Wind Tunnel

8-ft TPT Langley 8-Foot Transonic Pressure Tunnel

TEST-PANEL SELECTION

A total of four separate areas on the bottom of the Shuttle were considered at various times for testing, but simulation concepts and hardware were developed for only three. Near the end of the program one of these was dropped, leaving the two panels which had previously been declared critical for the first flight to be tested. The latter two panels are located ahead of and behind the forward external-tank/ Shuttle-orbiter connection bipod (yoke), and are among the most critical panels on the Shuttle. Local laminar heating rates during entry are extremely high, and if the tile loads during ascent cause the surface to become rough, then premature transition to turbulent flow could occur. Consequently, the heat of entry is increased over the downstream areas, and the structural integrity of the orbiter could be jeopardized. The third panel, for which a simulation concept and hardware were developed, was located just ahead of the inboard aileron hinge and was chosen because of large predicted inplane deflections (which were later found to be overly conservative) and lift-off acoustic pressures. Sketches showing the location and geometry of the three panels are given in figures 1 and 2. Panel 20A, shown in the lower middle of figure 1 , is located about $2 \mathrm{ft}$ behind the bipod; panel $20 \mathrm{C}$ is just ahead of the bipod and includes a portion of the landing-gear doors; and panel 20D (fig. 2) is positioned at the rear of the wing ahead of the elevon hinge line. Panel 20D, which includes the tile array in the "test area" in figure 2, has a width of 49 in. and a length of 65 in. A fourth panel (20B), which is shown on the bottom right-hand side of figure 1, was dropped early in the program, because the ascent environment for panel $20 \mathrm{~A}$ was considered to be a more severe one.

Panel 20A was selected because of the high buffet loads induced by the unsteady wake of the bipod, the high vibroacoustic loads, and the possibility of a "step and gap" problem between the tiles due to the possible loosening of tiles. The structure in this area is of skin-stringer construction and was reproduced in the construction of panel 20A as shown in figure 3. The panel ahead of the bipod (20C) includes, as noted previously, a portion of the doors of the forward landing-gear compartment. These doors are constructed of a thick honeycomb sandwich material (see fig. 4) and 
are so rigid that structural vibrations were not a concern. However, buffet loads due to extremely high unsteady-pressure levels caused by the bow shock ahead of the bipod at supersonic speeds were a concern.

\section{SHUTTLE TRAJECTORIES}

The level and distribution of static pressures over the orbiter, the intensity and distribution of dynamic pressures, and the position and strength of shocks all depend on free-stream Mach number and static-pressure levels provided by the trajectory as well as vehicle attitudes (angles of attack and sideslip) which result from control inputs and winds. Over the years, prior to the STS-1 flight, hundreds of trajectories were postulated to examine the effects of various wind profiles, payloads, orbital parameters, and uncertainties in thrust levels and aerodynamic coefficients. For the CLOT, the time-varying environments were based on the STS-1 nominal-trajectory quantities shown in figure 5. Note that time starts at 30 seconds in this plot, which corresponds to a Mach number of 0.6 , a dynamic pressure of 350 psf, and a static pressure of 11 psi. Dynamic pressure reaches a maximum of approximately 580 psf at 45 seconds and remains nearly constant for the next 25 seconds. It was assumed in the CLOT program that trajectories with higher or lower maximum dynamic pressure could be simulated in the wind tunnel by raising or lowering the pressure level (total head) at which the test was run.

The symbols in fiqure 5 represent the values of $p_{\infty}, a_{\infty}$ and $M_{\infty}$ actually obtained in the flight of STS-1. Static pressure was slightly overpredicted for early times and underpredicted for later times; Mach number was in excellent agreement up until about 45 seconds, when flight values began to exceed those of the predicted nominal. These small differences in Mach number are amplified into larger differences in dynamic pressure by the dependence of dynamic pressure on Mach number squared. The agreement between the predicted and flight trajectories shown in figure 5 clearly indicates that simulations based on the predicted STS-1 nominal trajectory were well-founded.

\section{PRESSURE, DISTRIBUTIONS}

A number of wind-tunnel tests were carried out by the Shuttle Project on pressure models of the Shuttle orbiter and ascent configuration. However, most were conducted for the purpose of obtaining structural loads, and not TPS tile loads. The latter requires significantly more resolution, not only to obtain accurate pressure levels and gradients, but also for shock strengths and shock locations. At the start of the CLOT program, the only data available were from wind-tunnel tests denoted as IA105A, IA105B, and IA81. Because panel 20A was the first to be tested, and because it receives its highest loads at transonic speeds, tests IA105A and IA81 were of prime interest as they included transonic data. Another test (OA253) which included transonic data had been run, but the data were not yet available. Fiqure 6 shows centerline pressure distributions from these tests for Mach numbers from 0.6 to 1.4. It should be noted that the circle symbols, denoted as IA105A data, are not all actual data points, but points taken from previously established fairings of the data.

Concurrent with the panel-design and tunnel-modification activities early in the program, a series of transonic tests on subscale bipods were conducted in a small transonic wind tunnel. These tests were conducted to examine the severity of the blockage problem caused by the bipod at transonic speeds, and are discussed subse- 
quently. Of importance here is the fact that the pressure distributions obtained in this facility were markedly different from the faired curves of test IA105A. Numerous modifications to the setup were made in an attempt to match the IA105A data, but without success. Finally, a special effort was undertaken by Rockwell to provide OA253 data for comparison. Test OA253, which has much higher resolution in many areas on the Shuttle, was most helpful in resolving the dilemma. The large changes in the character and levels of pressure obtained in OA253, ahead of and behind the bipod, were in good agreement with the small transonic tunnel data. Thereafter, data from test OA253 were coupled with IA105A and IA81 data to provide a simulation standard. The solid lines in figure 6 are illustrative of the actual fairings used.

Even with the higher resolution of the OA253 test pressure data, many important features of the flow field could not be determined. In addition, detailed supersonic data are required ahead of the bipod for panel 20C, while OA253 tests were carried out only at transonic speeds. Variations of pressure in the lateral direction, separated-flow boundaries, and shock locations are all features of the flow field that existing data did not define with the precision required to assure an accurate simulation. Consequently, both transonic and supersonic tests of subscale bipods were undertaken. Results from these tests later proved indispensable. In subsequent paragraphs, the features of these additional tests are described.

\section{TRANSONIC TESTS OF SUBSCALE BIPODS}

As noted in the previous section, concern over the effect that the blockage of a full-scale bipod mounted in the 8-f.t TPT would have on the maximum obtainable Mach number led to the initiation of tests on subscale bipods in a small pilot transonic tunnel available at Langley. This facility is roughly a $0.22-$ scale model of the 8-ft TPT and has the same type of slotted test section and Mach number capability. The bipod models were either $0.22-s c a l e$, which provided the same ratio of bipod size to test-section size as the full-scale bipod in the 8-ft TPT, or 0.16-scale, which corresponded to a three-fourths scale bipod in the 8-ft TPT. Bipod legs of four different lengths were made for the $0.22-$ scale bipod base to provide varying amounts of blockage.

In addition to decreasing the length of the bipod legs to reduce the effects of blockage, a suction box was placed in the test-section plenum above the slots in the test-section ceiling. The box was roughly the same width as the test section, started at the leading edge of the slots, and covered about one-third of its length.

The subscale bipod tests were carried out using a false floor on the bottom of the test section in the same manner as proposed for the 8-ft TPT. The 0.22-scale false floor had a smooth upper surface and was installed to permit the floor boundary layer to pass underneath. Static-pressure taps were installed on the top and bottom of the false floor and on the sidewalls of the tunnel. The floor used in the 8-ft TPT tests contained a cavity for the tile array such that the top surface of the tiles was flush with the floor. It was also designed to act as a splitter plate so that the boundary layer on the floor of the wind tunnel could pass beneath the floor; however, this feature was eliminated early in the 8-ft TPT test program. Those portions of the floor ahead of and behind the segment containing the panel were designed to maintain as flat a pressure distribution over the panel area as possible.

Static-pressure distributions from the subscale tests were used for a number of purposes. They provided loads data on the false floor to facilitate the structural desion of the floor used in the 8-ft TPT, and they permitted an assessment of the 
effect of bipod length on pressure distributions, including shock locations. of equal importance was the fact that the distributions permitted an assessment of effectiveness of the suction box in reducing blockage effects. Finally, these tests permitted an early appreciation of the difficulties of obtaining the supersonic Mach numbers required for the aft bipod location of panel 20c. The data showed that the bipod legs could be significantly shortened without having a noticeable effect on the surface pressure distribution. The data also showed that the suction box enabled the tunnel to achieve a much higher effective free-stream Mach number. This latter benefit was only for the forward bipod location appropriate to panel 20A.

An example of the effect that the suction box had on the environment aft of the bipod is given in figure 7. Here, local Mach number is plotted as a function of distance along the false floor from the leading edge. The positions of the 0.22scale bipod and test panel are indicated. A large difference in the local Mach number levels on the panel between the no-suction and suction cases is apparent. Also apparent is the difficulty inherent in these tests (as well as those in the 8-ft TPT) of determining the effective Mach number of the onset flow. Ahead of the bipod, the flow stagnates on the leading edge of the false floor, then accelerates until it separates ahead of the bipod. Mach numbers, calculated using the local static pressure, decrease through the separated region and go to zero at the face of the bipod. Immediately aft of the bipod, there is a rapid flow expansion followed by a gradual compression. The flow beyond the flat segment occupied by the panel continues to decelerate, but does not quite reach its free-stream Mach number before the end of the floor is reached. In short, there is no flow in the test section corresponding to a uniform free stream.

Without suction (fig. $7(a)$ ), the flow accelerates behind the bipod to a Mach number of 1.1, which corresponds roughly to a free-stream Mach number of 0.7 . With suction (fig. $7(b)$ ), the local Mach numbers are comparable to those for a free-stream Mach number of 0.83 . This increase in effective Mach number due to suction is typical and permitted a good simulation of the flow behind the bipod up to free-stream Mach numbers slightly greater than 1.0 .

\section{SUPERSONIC TESTS OF SUBSCALE BIPODS}

The main source of loads of panel $20 \mathrm{C}$ was the unsteady pressure under the bow shock ahead of the Shuttle bipod. The magnitude of these loads varies directly with the strength of the shock and is a maximum for free-stream Mach numbers approaching 2.5. However, the Mach number just ahead of the bipod MONSET governs the bow-shock strength, and this is the flow condition that must be simulated in the tunnel. Because it was expected that the highest Mach number achievable in the 8-ft TPT would not be much in excess of 1.5, and this with a subscale bipod, a large amount of additional information had to be developed to formulate an acceptable simulation.

The 0.22-scale bipod employed in the pilot transonic facility was mounted on a flat plate and tested in the Langley Unitary Plan Wind Tunnel (UPWT) over a range of Mach numbers from 1.5 to 2.5. Surface pressures (on the plate) were measured to define the flow-separation point (see fig. 8) as well as the distribution of pressure ahead of the bipod. In addition, schlieren and shadowgraph photographs were obtained to define the average position of the bow shock. With these data, the position of the half-scale bipod (the size finally used) in the 8-ft TPT tests could be prescribed as a function of time, so that the bow shock could be positioned in the same location as that for a full-scale bipod. 
Pressure taps were located along the centerline ahead of the bipod and along rays $30^{\circ}$ and $60^{\circ}$ off the centerline. A plot of all the centerline pressure data is given in figure 9 in nondimensional form. The quantities $P_{S C A L E}$ and $x_{\text {SCALE }}$ are simple empirical functions of plateau pressure, onset Mach number, and bipod geometry, which were developed to correlate the data in a manner which would permit straightforward interpolations and extrapolations. The plateau pressure PPLATEAU is defined as the maximum pressure just downstream of flow separation. In figure 9, this would be the pressure at $x_{\operatorname{SCALE}}\left(\frac{x^{\prime}}{D}\right) \approx 4.0$. One of the primary parameters in the correlation expressions is the ratio of PPLATEAU to PONSET, which is itself defined by a simple empirical equation developed during this study. The accuracy of this simple equation, identified in figure 10, can be judged by the agreement with data plotted in the figure. Not only do the present data agree well, but those from a number of forward-facing-step experiments ranging in Mach numbers from 0.6 to 6.0 also agree well.

With the correlation equations for PSALE' $_{\text {SCACALE' }}$ and $\mathrm{P}_{\text {PLATEAU }} /$ PONSET $_{\text {STET }}$ available, the pressure distribution for any size bipod can be calculated given the onset Mach number and pressure. The application of these correlation relations to the generation of flight shock strengths $(\approx 3.78$ psi at MONSET $=2.1)$ with subscale bipods at lower Mach numbers (in this case, half-scale, two-third-scale, and threeguarter-scale bipods at ${ }^{\mathrm{M}}$ ONSET $\left.=1.65\right)$ is illustrated by the pressure distributions in figure 11. Note the increase in total-pressure head required at the lower Mach number to achieve the desired shock strength. Obviously, the levels of static pressure are also increased and flow separation occurs closer to the bipod. Figure 12 can be used to illustrate this effect moxe graphically. It shows the flow-separation boundaries for a full-scale bipod at the free-stream conditions that provide the maximum shock strength compared with that for a half-scale bipod at representative wind-tunnel conditions. The half-scale bipod is positioned to give the same howshock locations as the full-scale bipod. A better understanding of the separatedflow region and the associated surface streamline configuration can be obtained from figure 13. Shown here are oil flows for Mach numbers of 1.5, 1.8, and 2.1, with an outline of the panel boundaries superimposed. These photographs show not only the reverse flow ahead of the bipod, but also an interesting wake flow configuration inside a recompression shock.

Another valuable piece of diagnostic information obtained from the UPWT tests was the measurement of the detachment distance of the bow shock on the bipod as a function of Mach number. Both schlierens and shadowgraphs were obtained; an example of the latter is given in figure 14 for a Mach number of 2.1. The variation of shock-detachment distance (measured at the top of the bipod flange located near the bottom of the bipod) with Mach number is shown in figure 15. These data were useful for two very distinct purposes: (1) they provided the information needed to move a subscale bipod so that its bow shock could be located in the same place (as a function of time) as that for the full-scale bipod, and (2) they provided another method of estimating the effective free-stream conditions in the 8-ft TPT where there were significant Mach number gradients ahead of the panel. Given that the important tileload contributors are pressure gradients (especially those associated with shocks), time-varying pressure levels, and unsteady pressures associated with flow separation and shock movement, it is clear from figures 11 and 12 that the use of a half-scale bipod significantly reduced the area over which realistic tile loads can be simulated. It should also be pointed out that when the total-pressure head of the onset flow is raised to provide the same bow-shock strength for a half-scale bipod at an onset Mach number of 1.65 as that obtained in flight for an onset Mach number of 2.1, 
then the oblique shock from the leading edge of the separated-flow regions (see fig. 8) is much stronger than that encountered in flight at the same conditions.

\section{TIME-VARYING ENVIRONMENTS}

The time-varying free-stream conditions of figure 5 can be used with the pressure distributions obtained in the wind-tunnel tests of IA105A and B, IA81 and OA253, and on the subscale bipods at transonic and supersonic speeds to define the pressure distributions around the bipod at specific points in time. These data can also be used to provide a continuous time history of the static pressure, dynamic pressure, and Mach number at a specific point on the surface. If one is reasonably sure, on the basis of favorable comparisons of pressure distributions obtained in complete model tests with those obtained in the wind tunnel where the bipod was the dominant feature, that the latter provides accurate pressure distributions, then time histories at a single point can be used to effect the simulation of pressures for a much larger area. This is the situation that existed following the completion of the subscale bipod tests; consequently, most of the time simulations were done on the basis of those measured at a single representative point. For panel 20A, this point was at three-fourths of the panel length from the leading edge and on the centerline of the panel. This was where an actual data point (rather than faired data) was available and where there were both static- and dynamic-pressure taps on the calibration panels used to perfect the simulation in the 8-ft TPT. The point chosen for panel $20 \mathrm{C}$ was $12 \mathrm{in}$. ahead of the bipod, where both unsteady- and steady-pressure data were available from the calibration panels.

The application of the "point" data for panel $20 \mathrm{~A}$ was different from that of 20C. As noted in the "Introduction," the loads of concern on panel 20A were due to flow separation behind the bipod and associated unsteady pressures (buffet loads). These pressures tend to scale with local dynamic pressure, but are also dependent on local Mach number because of "zone-of-dependence" considerations. For panel 20C, the main concern was the large unsteady pressures in the separated-flow region, particularly those close to the bipod under the bow shock. Local static and dynamic pressures ahead of the supersonic bow shock must be generated in the simulation that produces the same shock strength as in flight.

The variations with time of dynamic pressure and Mach number at the threequarter-panel-length point for panel $20 \mathrm{~A}$ are given in figure 16. Also given in this figure for comparison is the time variation of free-stream dynamic pressure with Mach number. It is clear from figure 16 that neither dynamic pressure nor Mach number at the point vary with time in the same way as the free-stream quantities. Point dynamic pressure $q_{2}$ peaks earlier and at a higher level than the free-stream dynamic pressure; point Mach number $M_{l}$ is higher than the free-stream values in the transonic speed range, a totally expected result based on the pressure distributions of figure 6. Variations of the flow parameters with time ahead of the full-scale bipod are plotted in figures 17 to 20. Free-stream onset and point values for a point $24 \mathrm{in.}$ ahead of the bipod in the region of the pressure plateau are presented. The Mach number at this point increases continuously with time, but at a lower rate than in the free stream. Dynamic pressure on the other hand has two maxima, one at 45 seconds and another at 81 seconds. Note that the second maximum is much larger than the first, and occurs at a free-stream Mach number near 2.0.

Figure 19 is presented to show the variation with time of the pressure jump across the bow shock ahead of the bipod as well as the pressure jump across a normal shock with $M_{\infty}$ as the onset Mach number. The quantity $\Delta p_{S H O C K}$ peaks at a value of 
3.82 psi at 90 seconds into the flight, where the onset Mach number is 2.1 and the free-stream Mach number is 2.5. Some appreciation for the strength of the bow shock can be obtained from the fact that in the transonic speed range no shocks stronger than 2.2 psi are expected. Shock-detachment distance as a function of time is given in figure 20. The shock first starts to form around 70 seconds and moves steadily closer to the bipod. At the time when the pressure rise across the shock is at the maximum, the detachment distance is just over 3 in.

\section{STRUCTURAL DYNAMICS OF PANEL $20 A$}

As noted in the "Introduction," the location of panel $20 \mathrm{~A}$ was chosen because of large predicted buffet and vibroacoustic loads. To insure that these loads were simulated as accurately as possible, the structural features of the Shuttle in the region of panel 20A were incorporated in the construction of the panel. The basic structure was formed using an aluminum skin with hat-section stringers in the stream direction located on 3.88-in. centers as shown in figure 3. Two major frames and one miniframe in the transverse direction completed the simulated shuttle structure which was mounted in an I-beam rectangular "picture" frame. The resulting size of panel $20 \mathrm{~A}$ is small compared with the area on the shuttle that it represents. Consequently, its natural dynamical properties were not the same as the corresponding area on the Shuttle itself, particularly at low frequencies. Because of the potential effect which low-frequency vibrations, say less than $100 \mathrm{~Hz}$, would have on tile motion and loads, some means had to be provided to induce the low-frequency energy into the system. This was accomplished in the tests by the installation of a hydraulic shaker mounted below the tunnel on a 60 000-pound reaction mass which was isolated from the tunnel structure with air bags. The shaker was connected to the panel at four points using a specially built yoke. Figure 21 is a photograph of this setup. The time history of the amplitude of the input random-spectra vibration was controlled by a computer and coordinated with the time history of the diffuser flap, which was used to control the free-stream conditions in the test section (see "Simulation Methodology" for more detail).

The shaker inputs were based on the results from a larger test panel (known as FFA-04) in a reverberant chamber and from modal tests conducted on Orbiter ov101. These modal data were useful in separating the realistic responses obtained in the FFA-04 tests from those that related to the fixture. Because the test panel had a first-mode natural frequency of $75 \mathrm{~Hz}$, the shaker system was constrained to provide energy up to approximately $55 \mathrm{~Hz}$. Below $55 \mathrm{~Hz}$, the energy input provides an inertial loading; consequently, the main focus of the simulation was an rms acceleration, and not spectrum shape. More details on the vibroacoustic and buffet simulations for panels $20 \mathrm{~A}$ and $20 \mathrm{C}$ are contained in a paper by Schuetz et al. (ref. 1).

\section{SPECIAL PROBLEMS IN PANEL 20C SIMULATION}

Because the free-stream Mach numbers required to generate the shock strengths (fig. 19), could not be achieved with a full-scale bipod in the 8-ft TPT, a great amount of difficulty was encountered in effecting an accurate simulation of the flow field over panel 20C. Even with a subscale bipod (or no bipod), there was never an expectation that the 8-ft TPT could be modified to achieve an onset Mach number of 2.1. The hope was that the right combination of bipod size and supersonic nozzle could be found to achieve an onset Mach number greater than 1.6. With this Mach number, the total-pressure head in the tunnel could be raised relative to that in flight so that the required shock strength could be obtained. The nozzle required to 
accelerate the flow to supersonic speeds was provided by building up the sides and ceiling of the tunnel with fiberglass-coated, contoured blocks in the throat region and z-bar-reinforced aluminum sheets upstream and downstream. This was to provide as gradual a contraction and expansion as possible. The sides of the nozzle diffuser extended downstream to about the middle of panel $20 \mathrm{C}$, while the top-side fairing terminated about a foot beyond the bipod. In the tunnel-empty configuration (with the bipod removed), the supersonic nozzle yielded an onset Mach number of close to 1.8. With two-third-scale and half-scale bipods (with truncated legs), the maximum Mach numbers achievable were approximately 1.35 and 1.65 , respectively. Clearly, the half-scale bipod had to be used to simulate the maximum shock jumps and associated unsteady pressures encountered in flight.

With the aid of the supersonic UPWT data previously described, time histories of onset Mach number and local values of static and dynamic pressure could be determined that would yield the required shock-pressure jumps. Since a half-scale bipod was employed in the tests, a point closer to the bipod than the $24 \mathrm{in}$. chosen for the full-scale bipod (figs. 11, 17, and 18) had to be used to monitor the time variation of $p_{2}, M_{1}$, and $q_{l}$. A point 12 in. ahead of the bipod was selected. Onset Mach number versus time is plotted in figure 22 for the $8-f t$ TPT simulation and for the Shuttle in flight. The variation of dynamic pressure with time at the 12-in. point for the 8-ft TPT simulation and the Shuttle STS-1 flight is given in figure 23. The Mach number short fall (1.65 versus 2.1) at the point of maximum shock strength requires that the wind tunnel be run at a total-pressure head of approximately $1.0 \mathrm{~atm}$ instead of the flight value which is slightly above $0.6 \mathrm{~atm}$. The adverse effect of running at this higher total head is that the point values of dynamic pressure (fig. 23) are much higher than the flight levels and persist for a longer time. This phenomenon stimulated a quick look at what happens when the dynamic pressure is matched (at the 12-in. point) instead of $\Delta \mathrm{p}_{\text {SHOCK}}$. The result is shown in figure 24 and indicates that a far weaker shock is generated than that desired and for a much shorter time.

It can be expected, with the use of a half-scale bipod and a maximum tunnel Mach number of 1.65, that the movement of the bow shock will be less than that for the full-scale bipod at flight Mach numbers. Furthermore, when the Mach number in the tunnel decreases for times greater than 90 seconds (in order to continue to match the STS-1 shock strength), then the bow shock moves away from the bipod instead of moving closer as it does in flight. The above deficiencies are abundantly clear in figure 25, which shows the STS- 1 shock standoff distances and those incurred for a fixed half-scale bipod. Early and late in the $\Delta \mathrm{p}_{\text {SHOCK }}$ pulse, the half-scale bipod shock position is in error by about $3 \mathrm{in}$. with a "zero error" at the crossover point at 92 seconds. The desirability of being able to move the bipod to position the shock in the "right" location is apparent. Consequently, the bipod was mounted on a hydraulic ram with a stroke of $6 \mathrm{in}$. The position of the ram was automatically controlled and coordinated with the diffuser-flap movement.

\section{TEST SETUP IN 8-FT TPT}

Figure 26 is a sketch of the tunnel test section and the false-floor test fixture. The tile arrays, as previously noted, fit into a cavity in the false floor so that their surfaces are flush with the floor surface. The bipod is positioned ahead of and behind the cutout, depending on which tile array is to be tested. In the sketch, the bipod is depicted in the position appropriate for panel 20A. A special suction hox was installed in the plenum atop the ceiling of the test section for tests of the panel aft of the bipod (panel 20A) in a way similar to that described 
for tests of the subscale bipods in the small pilot transonic tunnel. As noted for the small tunnel, this provided some relief from the choking effect of the bipod and permitted the simulation of a higher maximum Mach number. Tests of the panel forward of the bipod (panel 20C) required the use of a three-wall choke or nozzle, which was described in the preceding section. The suction box was not used in tests of panel 20C.

The time-varying free-stream conditions are provided by controlling the deflection angle of the diffuser flaps, shown in figure 26, in a prescribed way. When the tunnel is started, prior to the start of a simulation, the flaps are positioned at their full-in position, which is about $33^{\circ}$ from the wall. This flap position yields a test-section Mach number of about 0.6. Dynamic pressure and Mach number are increased by moving the flap toward the tunnel wall. Maximum conditions are usually achieved when the flap is within $5^{\circ}$ or $6^{\circ}$ of the wall; further decreases are ineffective. The maximum angular rate for moving the flap was about $1.5^{\circ}$ per second.

The false floor was originally constructed much like an airfoil with a flat bottom and was mounted on streamlined supports about 2 in. high. This permitted the onset boundary layer to pass beneath the floor. Consequently, the boundary layer just ahead of the panels was kept thin - about the same thickness as that on the Shuttle. Also, it was initially thought that the diffuser would operate more efficiently with the bypass and that the diffuser flap would be more effective in controlling the flow as well. It was found through trial and error after the floor was installed that the performance of the tunnel was, in fact, improved with the bypass closed. Further improvement in tunnel performance was achieved by the installation of vortex generators on the false floor several feet downstream of the panel trailing edge. Boundary-layer thicknesses were measured on the false floor with the bypass closed for both panel $20 \mathrm{~A}$ and $20 \mathrm{C}$ installations. The measurement for the former was taken about $1 \mathrm{ft}$ from the sidewall at the same longitudinal location as the bipod. For $20 \mathrm{C}$, the measurement was $4 \mathrm{in}$. from the centerline and $1 \mathrm{ft}$ downstream of the leading edge of the panel. The boundary-layer thickness ranged from 1.0 to $1.25 \mathrm{in}$. for panel $20 \mathrm{~A}$ and 0.5 to $0.7 \mathrm{in}$. for panel 20C. These values are in good agreement with those predicted for the shuttle under similar conditions.

Figures 27 and 28 are photographs of the test panels installed in the false floor. The photograph of panel 20A ( $\mathrm{fig}$. 27) was taken looking upstream. Visible on the left-hand side of the photograph are total head probes mounted on the sidewall of the tunnel. A flashbulb compartment is also visible just downstream of the panel which was necessary to provide a time-coordinating flash for the various motionpicture cameras. The photograph of panel 20C (fig. 28) was taken looking downstream. Faintly visible in the background are the diffuser flaps at the downstream edge of the false floor. The housing aft of the bipod is for the hydraulic ram used to translate the bipod.

FEATURES OF CALIBRATION AND TEST PANELS

The original plan was to precede each "real" panel test by tests of two calibration panels. One panel, referred to as the precalibration (precal) panel, was wooden and was instrumented with a large number of static-pressure orifices and a few Kulites (microphones). The second, simply known as the calibration panel, had realistic structure and, except for a few instrumented real tiles, was covered with foam tiles. A large number of Kulite gages (capable of both absolute and dynamic-pressure measurements), static-pressure orifices, accelerometers, and proximity gages were installed on the calibration panels for measuring the time-varying environment and to 
obtain panel and tile response data. The wooden precal panel was used to perfect the simulation in terms of the pressure environment. Steady-state pressure distributions measured for specific discrete flap angles or quasi-steady distributions obtained during a dynamic run at specified intervals of time ( 5 or 6 seconds) could be obtained. For panel 20A, both calibration panels were tested prior to testing the real panel, while for panel $20 \mathrm{C}$, the real panel was tested after the wooden precal panel.

Figure 29 is a sketch of the types and locations of instrumentation on the calibration panel for panel 20A. There are a total of 191 sensors, including 109 Kulites, 51 static-pressure taps, 15 strain gages, and 16 accelerometers. Most of the gages, of course, are surface sensors, but there are a few static-pressure and Kulite gages under the tiles or on their sides (fig. 29(a)). Accelerometers and strain gages are located on the bottom of the panel and are distributed as shown in fiqure 29(b). A few of these same locations on the test panel are instrumented with additional strain gages on the top side of the bottom cover as indicated by the asterisks. The calibration panel for panel $20 \mathrm{C}$ has fewer total instruments than panel 20A. This is partly because of the limited interests in the structural dynamics of the panel and the more localized nature of the loads. It has 94 Kulite gages, 39 static taps, 4 strain gages, and 8 accelerometers.

Both calibration panels were equipped with specially instrumented real silica tiles which were used to obtain tile response and loads data. In panel 20A, this tile is located about two-thirds of the length along the panel and is outlined with a heavy line in figure 29(a). Panel $20 \mathrm{C}$ has two instrumented tiles; one is in the first row of tiles on the door and to the right of the bipod, and the second is to the left of the centerline in the second row of tiles on the door. Part of the special instrumentation was the installation of mini coe sensors (ref. 2) distributed in three rows on the bottom of the tile. These sensors consist of a strain gage bonded to a diaphragm, which in turn is bonded to the bottom side of the adhesive which holds the strain isolation pad to the aluminum surface. Once installed, the face of the diaphragm is flush with the aluminum surface. Normal force, pitching and rolling moments which are applied to the tile can be determined through an appropriate calibration of the sensor outputs (ref. 3).

Figures 30 and 31 are sketches of the real, or test, panels for configurations 20A and 20C, respectively. Fiqure 30 (panel 20A) shows that there is still a large number of sensors (184) employed, but most of these are located on the inner mold line (IML). Several tiles are instrumented internally with accelerometers to obtain response data. The area inside the heavy line is covered with real 9-PCF (pounds per cubic foot) densified tiles on a $0.160-i n$. SIP. On the outside, the surface is covered with 9-PCF polyurethane foam on a 0.160 sIP. Three tiles contain diagnostic flight instrumentation (DFI), just as they do on the Shuttle, to determine if the installation of the instrumentation causes any degradation in the tile performance.

The general arrangement for panel $20 \mathrm{C}$ is shown in figure 31 . As on panel 20A, panel $20 \mathrm{C}$ is covered with both silica tiles and polyurethane foam. Only three rows of real tiles were installed on panel 20C, and these are contained within the heavy dark line in the figure. The first row of tiles on the doors and those adjacent to the center edge of the door are comprised of 22-PCF silica tiles; those outside these regions on the second and third rows are 9-PCF densified tiles. Two of the real tiles contain DFI and are so labeled. 


\section{SIMULATION METHODOLOGY}

In flight, the dynamic pressure at any point on the shuttle increases, reaches a maximum, and then decreases, while Mach number continues to increase up to orbital values. The curves in figures 16, 17, and 18 showing the variations with time of $q_{q}$ and $M_{2}$ ahead of and behind the bipod depict this situation. The Mach number capability of a transonic wind tunnel, on the other hand, is limited to low supersonic speeds, and the speed must go up or down to cause the dynamic pressure to go up or down. Consequently, when the simulation of flight conditions requires dynamic pressure to decrease after reaching its maximum, then tunnel Mach number must also be reduced. The dynamic-pressure simulation is, therefore, more accurate than that for Mach number following peak-load conditions.

\section{Panel 20A Simulation}

Prior to starting the simulation process, it is necessary to define the timevarying environment to be simulated. The procedure for doing this is discussed in a preceding section, where it was shown that by using static data for various windtunnel tests, the time variations of dynamic pressure and Mach number at any point on the panel could be determined. For panel 20A, a point 52 in. downstream of the leading edge of the panel was chosen as the "representative" point. Results from this process are plotted in figure 16 and are repeated in the upper left-hand corner of figure 32. It should be noted that when the flight flow variables are matched at the representative point, then they are also in good agreement in the surrounding area.

The problem now is to determine the diffuser flap-angle time histories that will give a good approximation of these time variations. To accomplish this, a series of runs are made at discrete flap-angle settings to construct a curve such as the one shown schematically in the upper right-hand corner of figure 32 and guantitatively in figure 33. Each value of $q_{2}$ or $M_{2}$ can be related to a flap angle and to time. Consequently, the flap-angle time history needed to yield the dynamic-pressure time history (upper left-hand corner) can now be constructed. The sketch in the lower right-hand corner of figure 32 depicts this result. A dynamic run is then made with the calculated flap time history, and the resulting dynamic-pressure (and Mach number) time history ${ }^{1}$ is compared with that desired. If the simulation is not satisfactory, the flap-angle time history is adjusted, taking into consideration this new information, and the process is repeated. If the rate of change of the flight dynamic pressure requires a larger rate of change of the flap angle than the maximum available, $1.5^{\circ}$ per second, then the maximum rate is used until the flight and predicted values come back into agreement. Adjustments in the level of pressure can be made by changing the stagnation-pressure level at which the test is run.

Prior to making simulation runs, a series of tests were conducted to determine the time lags in the tunnel circuit, i.e., the time it takes a flap-angle change to be felt in the test section. Sawtooth time variations of the flap angle were used with several different amplitudes of the sawteeth. The lags determined using these

${ }^{1}$ Dynamic-pressure time histories were determined using static-pressure time histories obtained from absolute pressure Kulites. The calibration, data reduction, and plot programs for the Kulite pressures were formulated and implemented by $D$. R. Rummler of Langley Research Center. 
data were factored into the initial quess or first iteration of the flap-angle time history plotted in figure 34.

The "first-iteration" flap-angle time history provided a dynamic-pressure pulse that was too narrow and did not obtain the maximum value required. It was thought that the maximum could be increased by permitting the flap to remain at its lowest angle for an additional 2 seconds. In addition, by delaying the rest of the flapangle time history by this same 2 seconds, it was hoped that the dynamic-pressure pulse would be broadened sufficiently. The flap-angle time history incorporating these changes is labeled "second iteration" in figure 34. Figure 35 shows the variation of dynamic pressure obtained using this flap-angle unit. Clearly, the maximum value of $\mathrm{g}_{2}$ and the character of its variation with time agree well with the predicted STS-1 curve. The simulated pulse is slightly broader than that for STS-1, but the differences are small and are deemed acceptable.

Variations in Mach number at the representative point with time for the predicted STS-1 trajectory and in the wind tunnel using the second-iteration flap-angle time history are shown in figure 36. Simulated Mach numbers are larger than those expected for STS-1 prior to reaching the maximum Mach number $(1.2)$ in the tunnel simulation. Beyond this point, the difference between the simulated and predicted STS-1 local Mach number increases rapidly. Comparison of figure 33 with figure 36 indicates that the maximum Mach number achieved in the dynamic runs was slightly lower than that obtained in steady-state runs.

A fatigue scatter factor of 4 was applied to the tunnel simulations so that one mission was defined as 4 load cycles. Not all the missions were run at the same tunnel stagnation pressure; hence, local static and free-stream dynamic-pressure levels were not the same. One mission (4 load cycles) was run at a free-stream stagnation pressure of $0.85 \mathrm{~atm}$ to simulate STS-1 flight conditions. Twenty-one missions were run at $1 \mathrm{~atm}$, which corresponds to a maximum free-stream dynamic pressure of 775 psf. This was as close to the design-load conditions ( $\approx 820$ psf) as the tunnel could provide.

Panel 20C Simulation

The simulation procedure for panel $20 \mathrm{C}$, as for $20 \mathrm{~A}$, was perfected using the wooden precal panel. The plateau pressure, at a point 12 in. ahead of the face of the bipod legs, and the onset, total, and static pressures were measured and used to calculate the $M_{1}, q_{2}$, and $\triangle p_{S H O C K}$ time histories for comparison with those shown in figures 17, 18, and 19. Shock-detachment distances were also measured in the 8-ft TPT precal tests and compared with those measured on $0.22-$ scale bipods in the subscale tunnel experiment to determine an effective onset Mach number. In addition, measured plateau and onset pressures were used in the correlation equation given in figure 10 to calculate onset Mach number as a check on the values determined from shock-detachment-distance measurements.

Figures $37(a)$ and $37(b)$ are shadowgraph and schlieren photographs for a Mach number of approximately 1.6 taken in the UPWT and the 8-ft TPT, respectively. A corner fillet obscures the flow at the bottom of the bipod in the 8-ft TPT schlieren. As previously indicated, the shock-detachment distances in the 8-ft TPT schlieren are about 3 in., which correspond to a Mach number of approximately 1.6 in a half-scale bipod. The maximum Mach number attained in the $20 \mathrm{C}$ simulation was 1.66 . This was based on plateau pressures and was roughly confirmed by schlieren photographs. 
The iterative procedure for defining the flap-angle time history required for panel $20 \mathrm{C}$ is similar to that depicted for panel $20 \mathrm{~A}$ in fiqure 32. The main difference is that the bow-shock pressure jump $\Delta \mathrm{p}_{\mathrm{SHOCK}}$ was the primary quantity required for simulation rather than $q_{2}$. Three iterations of flap-angle time history were

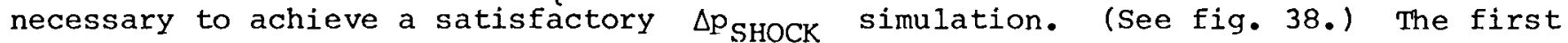
iteration shown in figure 38 yielded a $\Delta \mathrm{p}_{\mathrm{SHOCK}}$ pulse that was much too broad with a maximum $\triangle \mathrm{p}_{\mathrm{SHOCK}}$ of 3.63 . The second iteration gave rise to the $\triangle \mathrm{p}_{\mathrm{SHOCK}}$ pulse that was only slightly too broad but did not reach the desired $\triangle \mathrm{p}_{\mathrm{SHOCK}}$ Of 3.82 . The broadness of $\triangle \mathrm{p}_{\mathrm{SHOCK}}$ pulses was attributed, in part, to the inadequacy of the diffuser-flap drive motors to move the flap back out into the high-dynamic-pressure flow at the required rate. The third iteration in figure 38 was formulated with the hope that by bringing the flap back closer to the wall (between 85 and 90 seconds) and into the low-energy boundary layer, it would move away with more momentum and, consequently, maintain the desired rate of flap deflection through the higher flap angles. The desired width or broadness of the pulse was roughly maintained, but the maximum $\Delta p_{\text {SHOCK }}$ achieved was higher than required. No further improvements were attempted because of schedule commitments.

As noted in the key of figure 39, the data obtained in the tunnel simulations were translated 3.5 seconds in order to provide the best fit to the $\Delta \mathrm{p}_{\mathrm{SHOCK}}$ pulse. It was not necessary to modify the flap time history to adjust for this lag, since the primary concern was to have a realistic $\Delta \mathrm{p}_{\mathrm{SHOCK}}$ pulse during each load cycle. It should also be pointed out that the $\Delta \mathrm{p}_{\mathrm{SHOCK}}$ values plotted in figure 39 were derived using an onset Mach number based on the average shock-detachment distances measured from schlieren photographs for six of the early load cycles. A plot of this average curve is given in figure 40 and confirms that a maximum Mach number of approximately 1.66 was obtained.

Concern for the location of the bow shock on the panel ahead of the half-scale bipod was noted previously. A hydraulic ram was provided to translate the half-scale bipod in order to have its shock location match that of a full-scale bipod for as long as possible. The most complete match would have required the half-scale bipod to be moved forward of its normal position early in the $\Delta \mathrm{p}_{\text {SHOCK }}$ pulse; however, the unsteady-pressure levels just ahead of the bipod were found to be extremely high $(\approx 172 \mathrm{~dB})$, even though they dropped off rapidly. Therefore, it was decided not to move the bipod ahead of its normal position and put an unrealistically high load on those tiles immediately ahead of the bipod. Once the maximum Mach number was reached and the bow shock reached its most rearward position, with the bipod fixed, the bipod was translated rearward to keep the shock moving toward the rear, similar to flight, for as long as possible. The bipod was translated 2 in. (4 in. out of the 6 in. available was originally allotted to a planned forward translation) over a period of about 16 seconds and remained there for the remainder of the load cycle (fig. 41).

The variations of bow-shock location with time for six load cycles, relative to the position of the face of the fixed full-scale bipod, are bounded by the shaded band in figure 42. The data from seven of the earlier runs have been plotted at 3.5 seconds later, which is the same translation as that for the $\Delta \mathrm{p}_{S H O C K}$ pulse in figure 39. At times near 90 seconds, where $\Delta \mathrm{p}_{\mathrm{SHOCK}}$ is a maximum, the STS-1 curve and the experimental band cross. Overall, the agreement is good considering the limited translation employed.

Panel 20C was tested for 25 missions ( 100 cycles) using the setup described previously. At the start of the 26th mission, one of the supports of the top-wall nozzle fairing gave way. A number of attempts were made to restore the wall to its original configuration without tearing it down, but they were not successful. 
Finally, a new and more substantial top wall was installed; a number of unsuccessful adjustments were made in striving to achieve the performance of the original wall. In all, 14 missions ( 56 cycles) were run at maximum onset Mach numbers from 1.5 to 1.55 as various adjustments were made to the top wall. All the tests of panel 20C were carried out at a tunnel stagnation pressure of $1.0 \mathrm{~atm}$.

\section{UNSTEADY-PRESSURE ENVIRONMENTS}

Both panels $20 \mathrm{~A}$ and $20 \mathrm{C}$ have severe unsteady-pressure environments. The most severe environment for panel $20 \mathrm{~A}$ occurs at transonic speeds and is somewhat more familiar, in that most boosters incur buffet loads, of one kind or another, in this speed regime. The bow-shock-induced unsteady pressures on panel $20 \mathrm{C}$ are peculiar to configurations with attachment structures nearly normal to the flow. These pressures are not as well understood. Overall fluctuating pressure level (OFPL) data obtained in an investigation by Hanly (ref. 4) on a 0.035-scale model of the Shuttle ascent vehicle denoted (IS-2) provided the first quantitative results for the bipod area. Indeed, these data provided much of the justification for the panel $20 \mathrm{C}$ test.

Measurements of the OFPL on the calibration panel for 20 A ranged from 156 to $168 \mathrm{~dB}$. These levels were in good agreement with the levels measured in IS-2. Figure 43 shows the one-third-octave band $d B$ levels as a function of center frequencies for the CLOT panel 20A and for IS-2 tunnel tests as well as for the STS-1 flight test. Data are given for two longitudinal positions on the CLOT panel, one is $21.4 \mathrm{in}$. ahead of the trailing edge of the panel (fig. 43(a)), and the other is $10.55 \mathrm{in}$. from the trailing edge (fig. 43(b)). Both points on panel $20 \mathrm{~A}$ are $4.25 \mathrm{in}$. off the centerline. The precise location of the gages can be determined from figure 29(a). The STS-1 measurement on the shuttle corresponded to a point 32 in. downstream of the trailing edge of panel 20A. Exact coordinates for the IS-2 point are not known, but the gage was located in the same region that the CLOT pressures were measured.

One-third-octave band data for the simulated STS-1 nominal trajectory have been obtained for two 8-second time periods during the load pulse on panel 20A. The first period occurred prior to maximum load, and the second period brackets the time of maximum load as depicted in the small plot at the bottom of figure 43. Low-frequency shock motions occur in the region where the measurements were made during the latter part of the first 8-second period and then move off the trailing edge of the panel. Consequently, the spectra for the 8 seconds prior to peak loading, relative to that period around peak loading (square data points), contain much more low-frequency energy. The OFPL is not much different for the two time periods, as seen by the 0.4or $0.5-\mathrm{dB}$ differences in the levels noted in the figures.

Because the pressure gages for the Shuttle (STS-1) are 40 to 50 in. downstream of those on the CLOT panel, and since it is known from the IS-2 tests that the intensity of the pressure fluctuations diminishes downstream, a quantitative comparison of the CLOT and STS-1 curves should not be attempted. It is clear, however, that at low frequencies the CLOT data are higher than those for STS-1, a phenomenon that may be due to a significant reduction in shock activity and the flow becoming more uniform with increasing distance downstream of the bipod.

IS-2 data are lower than both the CLOT and STS-1 data at frequencies greater than $100 \mathrm{~Hz}$. At frequencies below $100 \mathrm{~Hz}$, IS-2 data are bracketed by the CLOT data from the two 8-second time segments. A more detailed comparison of the IS-2 and CLOT 
data could be useful in further defining the unsteady-pressure characteristics of the bipod wake.

Sound pressure levels encountered in front of the bipod on panel $20 \mathrm{C}$ are plotted in figure 44. Also shown are data points from the IS-2 tests and the STS-1 flight. Panel $20 \mathrm{C}$ and the IS-2 data indicate that the highest values of unsteady pressure are immediately ahead of the bipod and fall off rapidly with increasing distance from the bipod face. Ahead of the flow-separation line, which is roughly 24 in. ahead of the bipod face for a half-scale bipod, sound-pressure levels are negligible. The points taken from the IS-2 tests (complete model tests) show a lower OFPL away from the bipod, which probably is a reflection of the differences in local dynamic and static pressure (fig. 11) between the CLOT test and the full-scale bipod in flight. Also, the interaction of the oblique shock with the separated-flow wedge (fig. 8) gives rise to high sound-pressure levels. Since the oblique shock in the CLOT tests is much stronger than that for STS-1 because of the higher stagnation-pressure levels, the sound-pressure levels near the separation line tend to be higher than those for STS-1 in the same location. As noted in previous discussions (fig. 12), the separation zone and the associated oblique shock for a full-scale bipod would be much further forward on the panel than that for the half-scale bipod. Only one data point is available in this region from STS-1, and it is about $13 \mathrm{in}$. ahead of the bipod face. It is about $2 \mathrm{~dB}$ lower than the precal panel results.

The test panel for configuration $20 \mathrm{C}$ had a surface Kulite placed about 1 in. ahead of the bipod and a fraction of an inch off the centerline. Maximum rms pressure levels from a number of runs range from 170 to $173 \mathrm{~dB}$, which is in qualitative agreement with the precal panel results. The IS-2 point plotted at $x=3$ in. may be in better agreement with the CLOT results than is readily apparent if the averaging effect that the gage has in a high gradient flow is considered. The diameter of the Kulite gages used on the IS- 2 model, when scaled to full-scale dimensions, is approximately 6 in.

Generally, it is felt that close to the bipod, say the first 8 to $10 \mathrm{in}$. the CLOT environment was a good simulation of that provided by the STS-1 nominal trajectory. Further away, the rms pressure levels are 3 to $4 \mathrm{~dB}$ too high and correspond more to the tile design-load levels rather than to those encountered in the nominal STS-1 trajectory.

\section{EFFECT OF REPEATED LOAD PULSES ON CONDITION OF TILES}

Panel 20A was tested for 25 missions (or 100 load cycles), while panel $20 \mathrm{C}$ was subjected to 39 missions (156 cycles). Panel 20A, with its skin-stringer construction and separated-flow pressure environment, provided a very dynamic platform for its tiles. Motion pictures taken of the panel during a load cycle clearly show the overall motion of the panel due to the shaker inputs, as well as individual tiles moving in response to the vortex flow off the bipod. Movement of the tiles in several locations was large enough to cause contact with adjacent tiles. This contact resulted in the chipping of some tile coatings. The first chipping was noticed after five cycles; gradually, similar degradation appeared at other locations. The magnitude of the chipping can be seen in figure 45. The photograph was taken after 76 load cycles and clearly shows chipping at 4 or 5 different locations.

Even though there was some degradation of the coating of some tiles, the overall level of the step heights and gap widths between tiles remained about the same. In addition, no apparent loss of the structural integrity of the tile systems was incur- 
red. This judgment is based on the step and gap measurements, the similarity of tile motions throughout the tests, and the gentle pushing and shoving on individual tiles.

The tiles of panel 20C, which were mounted on a much stiffer structure, showed neither physical nor structural damage after 156 load cycles. Tiles on the third row on the door, which were subjected to much higher loads than those expected on a nominal-trajectory shuttle flight (and a full-scale bipod), showed no degradation. This increased load, as noted previously, was due to the close proximity of the separation line and associated oblique shock.

\section{CONCLUDING REMARKS}

The present paper describes the procedures and equipment used to provide a realistic time-varying environment for two arrays of shuttle tiles bonded to structures which accurately duplicate those on the shuttle. Special problems which were encountered because of the blockage of the bipod and the high Mach numbers required for panel $20 \mathrm{C}$ were overcome with the aid of additional supporting tunnel tests. Both panels were subjected to at least 100 load cycles with no apparent structural degradation, even though the panel aft of the orbiter-external-tank-connection bipod did incur some damage to the black coating of several of its tiles as a result of significant vibroacoustic and buffet loads. Since panel $20 \mathrm{~A}$ and panel $20 \mathrm{C}$ tests were first-flight critical, it was particularly gratifying during the tests that, after one Shuttle mission (4 load cycles), both panels appeared to be flawless.

Langley Research Center

National Aeronautics and Space Administration

Hampton, VA 23665

September 21, 1982

\section{REFERENCES}

1. Schuetz, P. H.; Pinson, L. D.; and Thornton, H. T., Jr.: Unsteady Environments and Responses of the Shuttle Combined Loads Orbiter Test. Shock \& Vib. Bull., Bull. 52, Pt. 2, U.S. Dep. Def., May 1982, pp. 157-163.

2. Coe, Charles F.: Buffet Loads on Shuttle Thermal-Protection-System Tiles. Shock \& Vib. Bull., Bull. 52, Pt. 2, U.S. Dep. Def., May 1982, pp. 147-155.

3. Rummler, Donald R.; and Pinson, Larry D.: Static Calibration for Measurement of Loads in Wind Tunnel Tests on Shuttle Surface Tiles. A Collection of Technical Papers - AIAA/ASME/ASCE/AHS 23rd Structures, Structural Dynamics and Materials Conference, Part 1, May 1982, pp. 374-380. (Available as AIAA-82-0754.)

4. Hanly, Richard $D_{.}:$Surface-Pressure Fluctuations Associated With Aerodynamic Noise on the Space Shuttle Launch Configuration at Transonic and Supersonic Speeds. Proceedings - AIAA/ASME/SAE 17th Structures, Structural Dynamics, and Materials Conference, May 1976, pp. 241-247. 


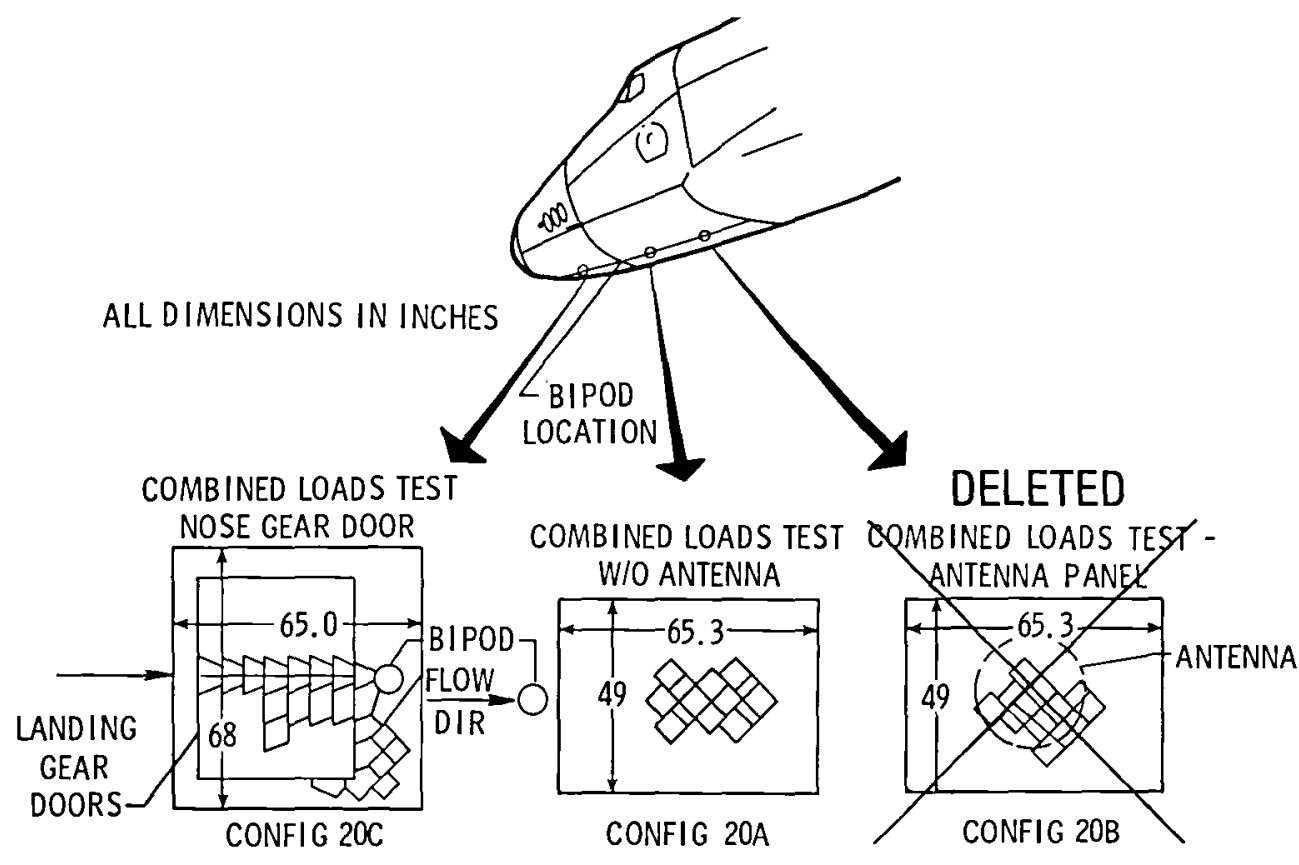

Figure 1.- Sketches showing locations and dimensions of panel configurations 20A, 20B, and 20C.

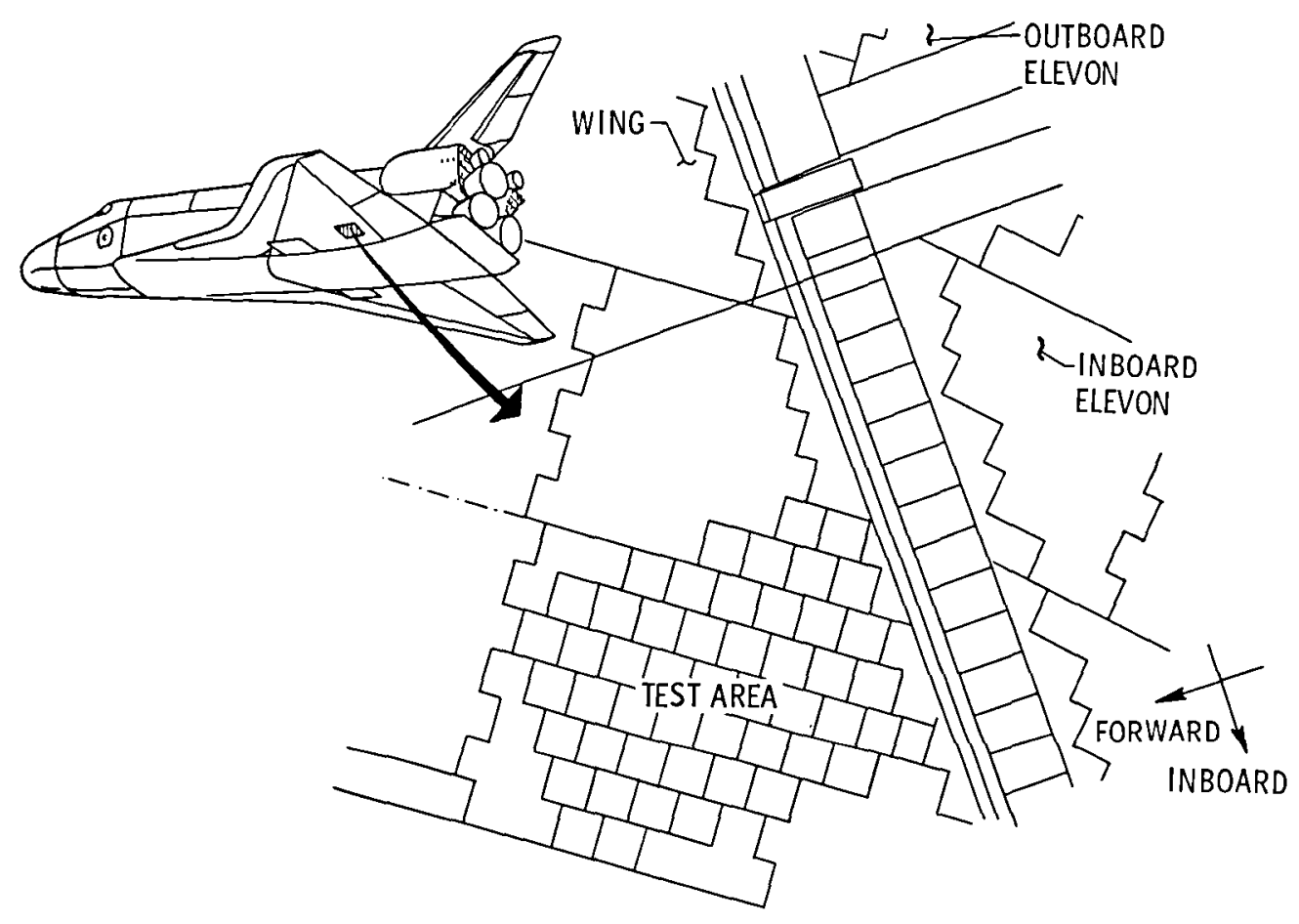

Figure 2.- Sketch showing location of panel 20D. 


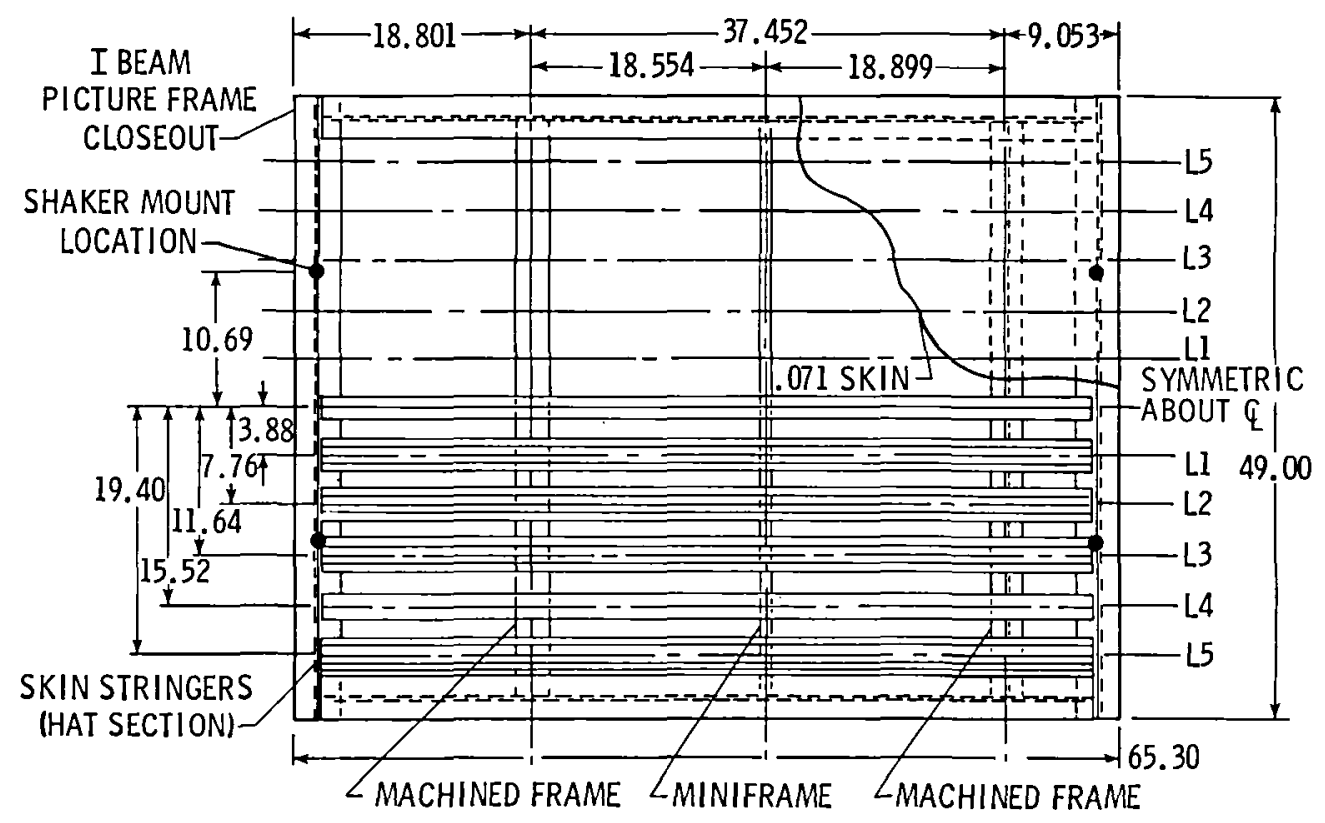

Figure 3.- Structural features of panel 20A. All dimensions are in inches.

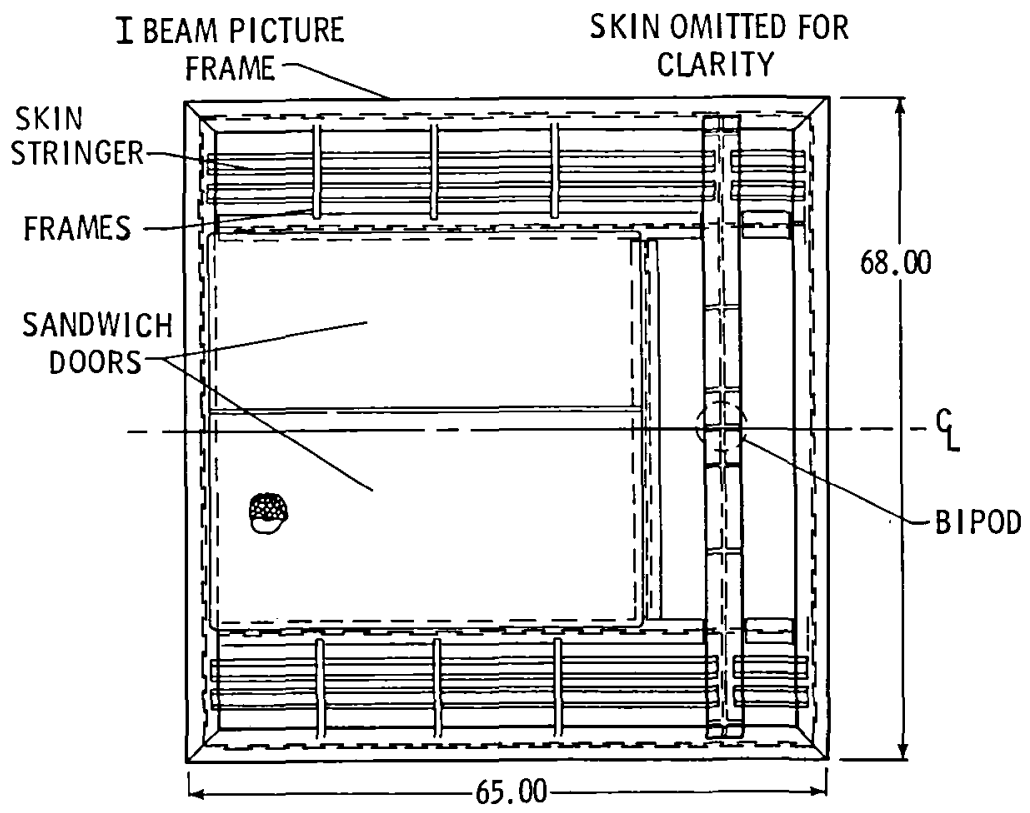

Figure 4.- Structural features of panel 20C. All dimensions are in inches. 


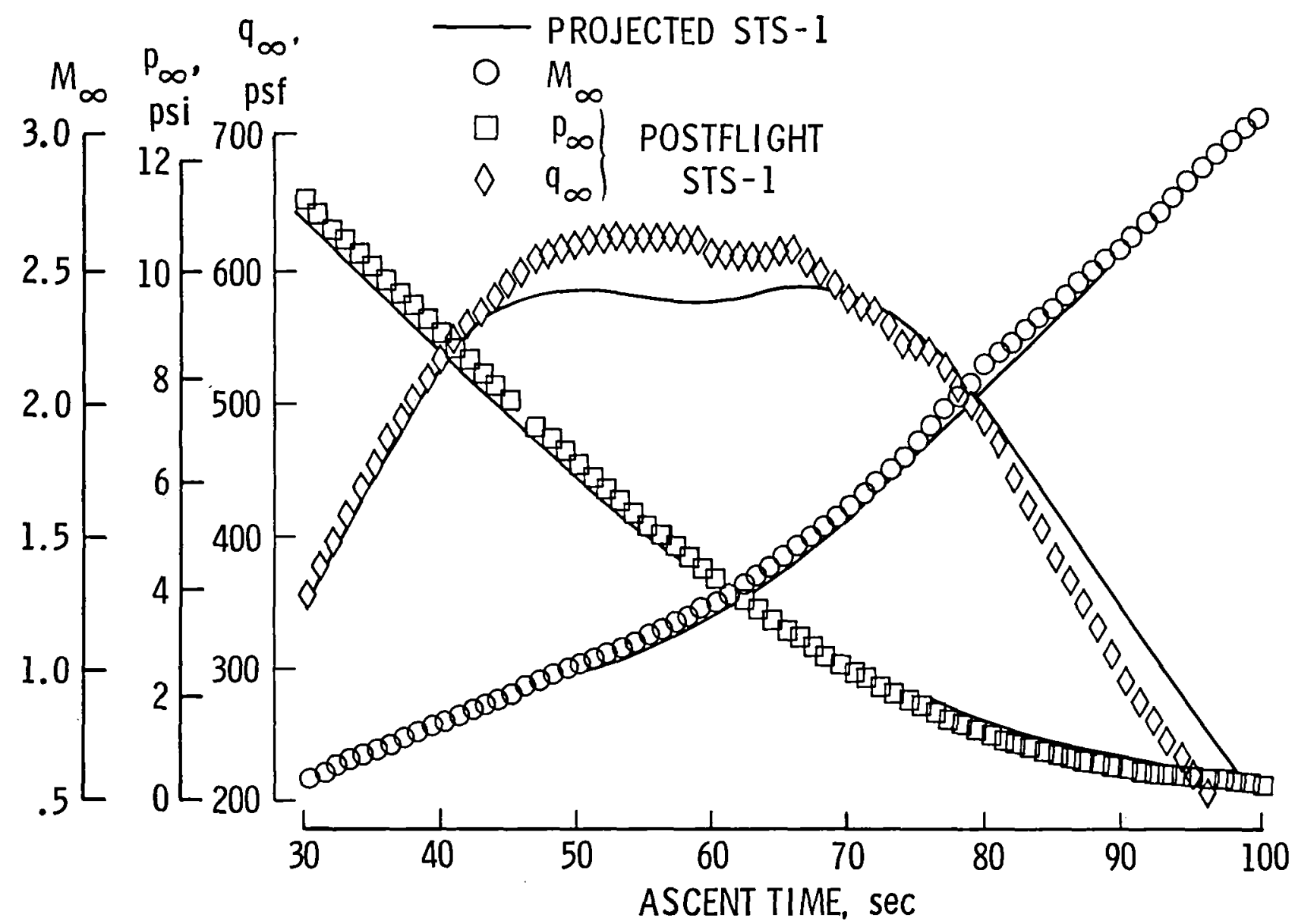

Figure 5.- Variation with time of free-stream Mach number, static pressure, and dynamic pressure for nominal STS-1 trajectory. 


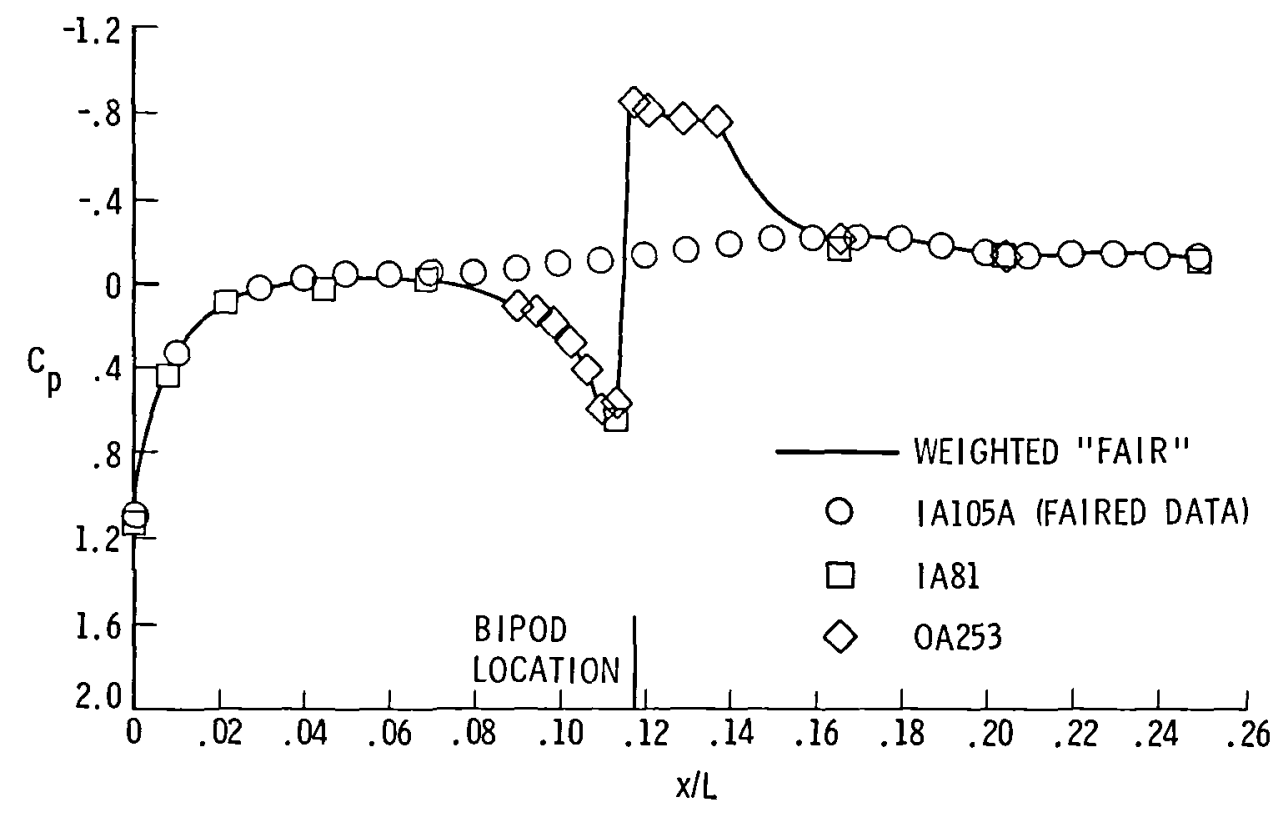

$$
\text { (a) } M_{\infty}=0.60 \text {. }
$$

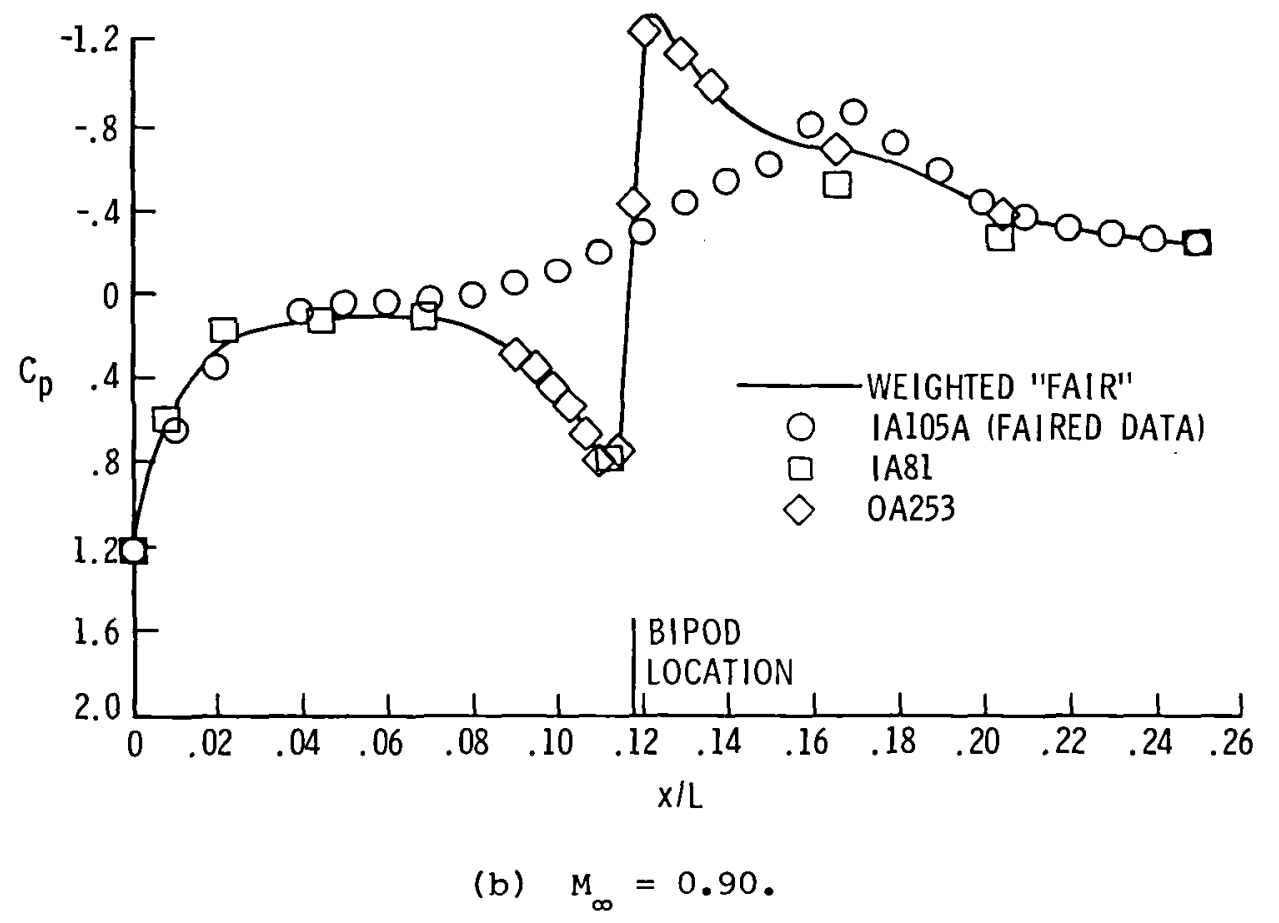

Figure 6.- Longitudinal variations of pressure along centerline of Shuttle for four Mach numbers. 


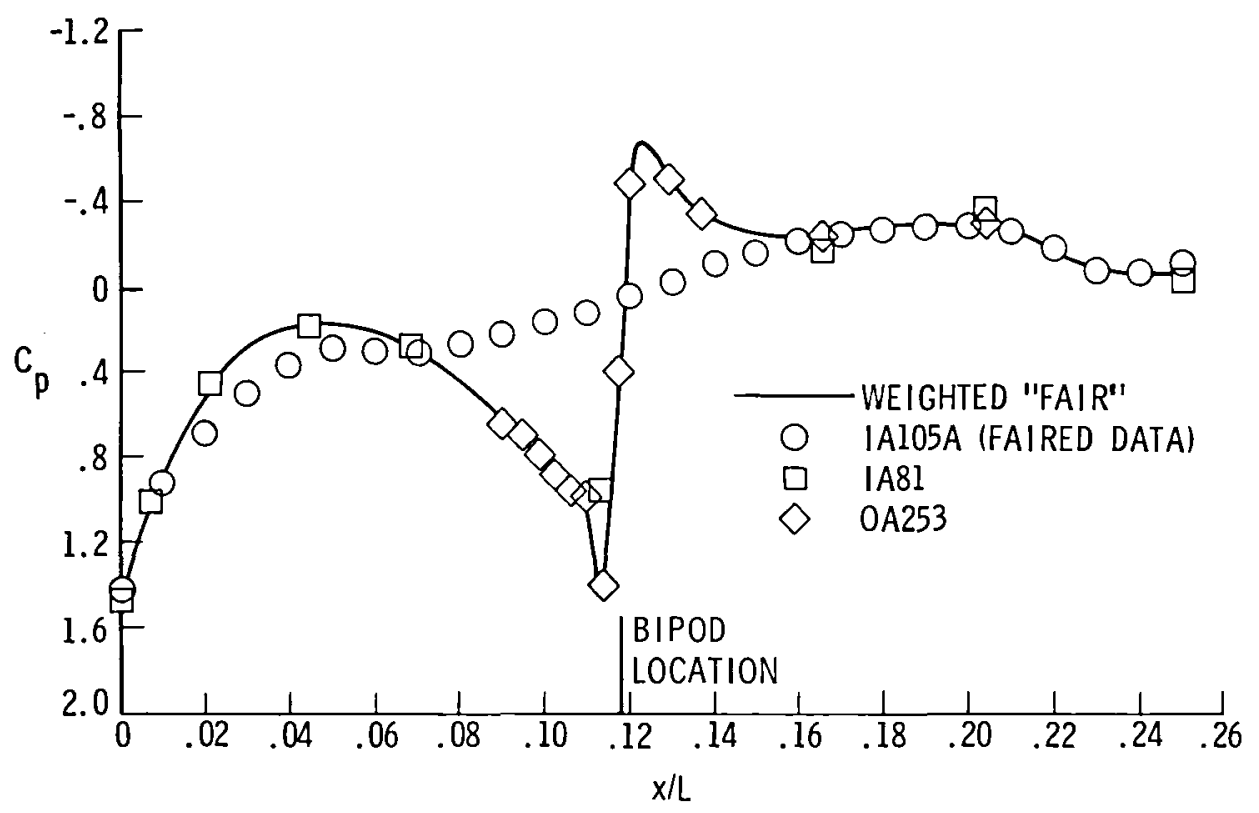

(c) $M_{\infty}=1.25$.

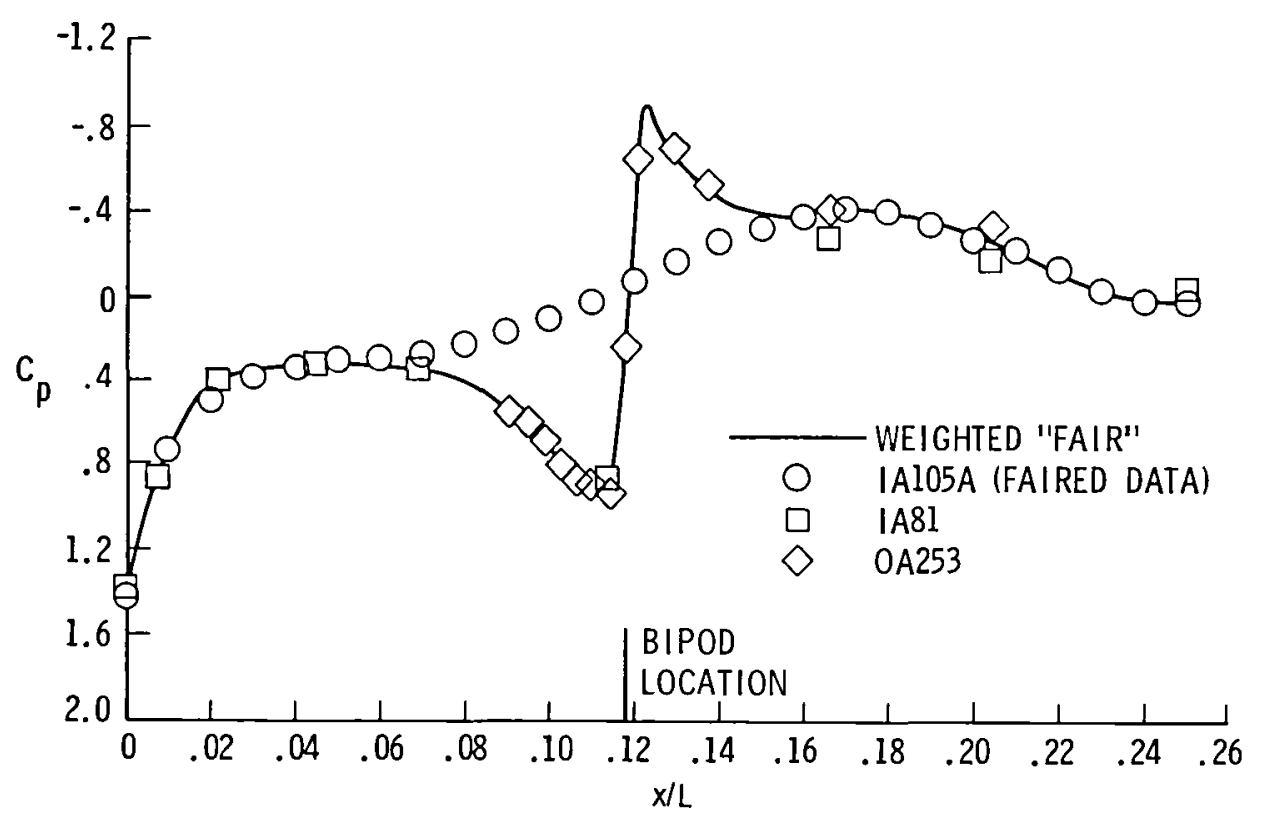

(d) $M_{\infty}=1.40$.

Figure 6.- Concluded. 


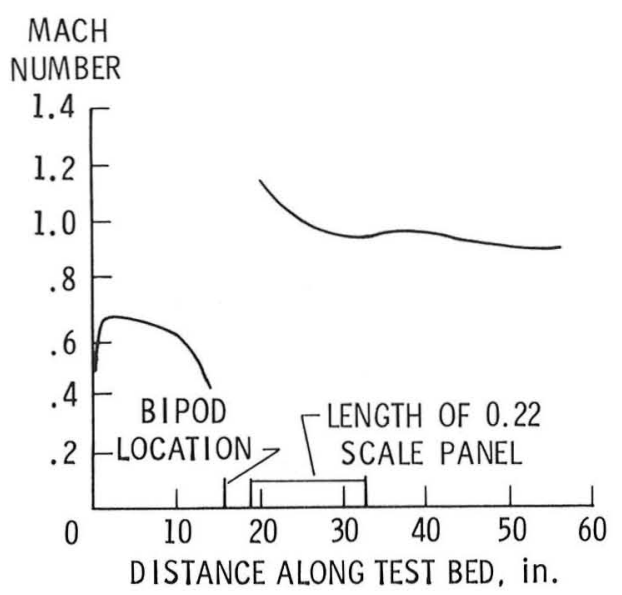

(a) No suction.

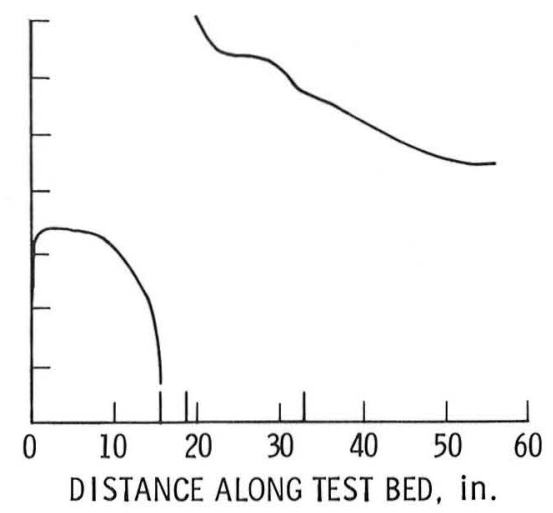

(b) Suction on ceiling of tunnel.

Figure 7.- Effect of suction on variation of local Mach number with longitudinal distance ahead of and behind bipod.
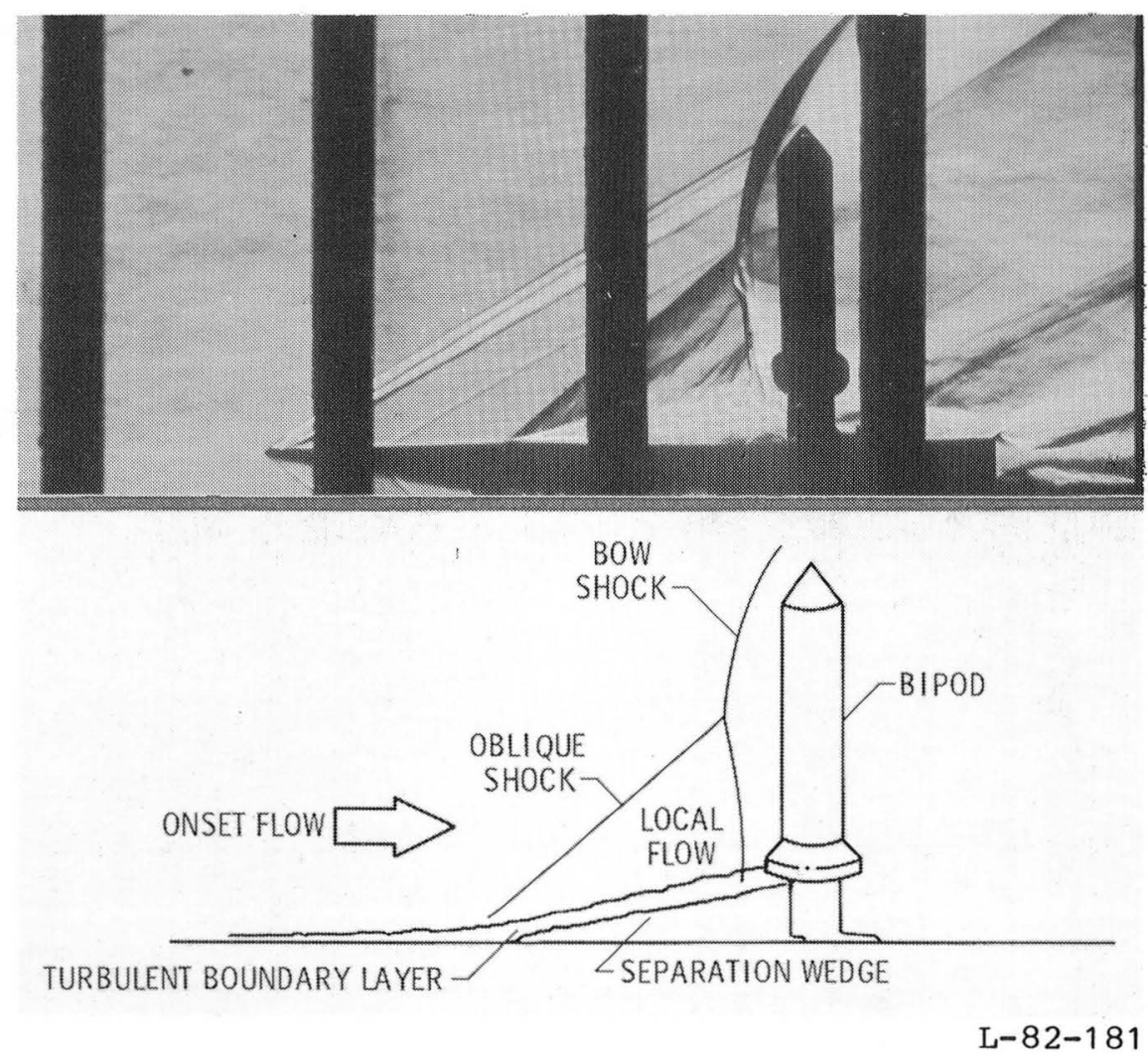

Figure 8.- Sketch and shadowgraph showing features of flow ahead of bipod. 


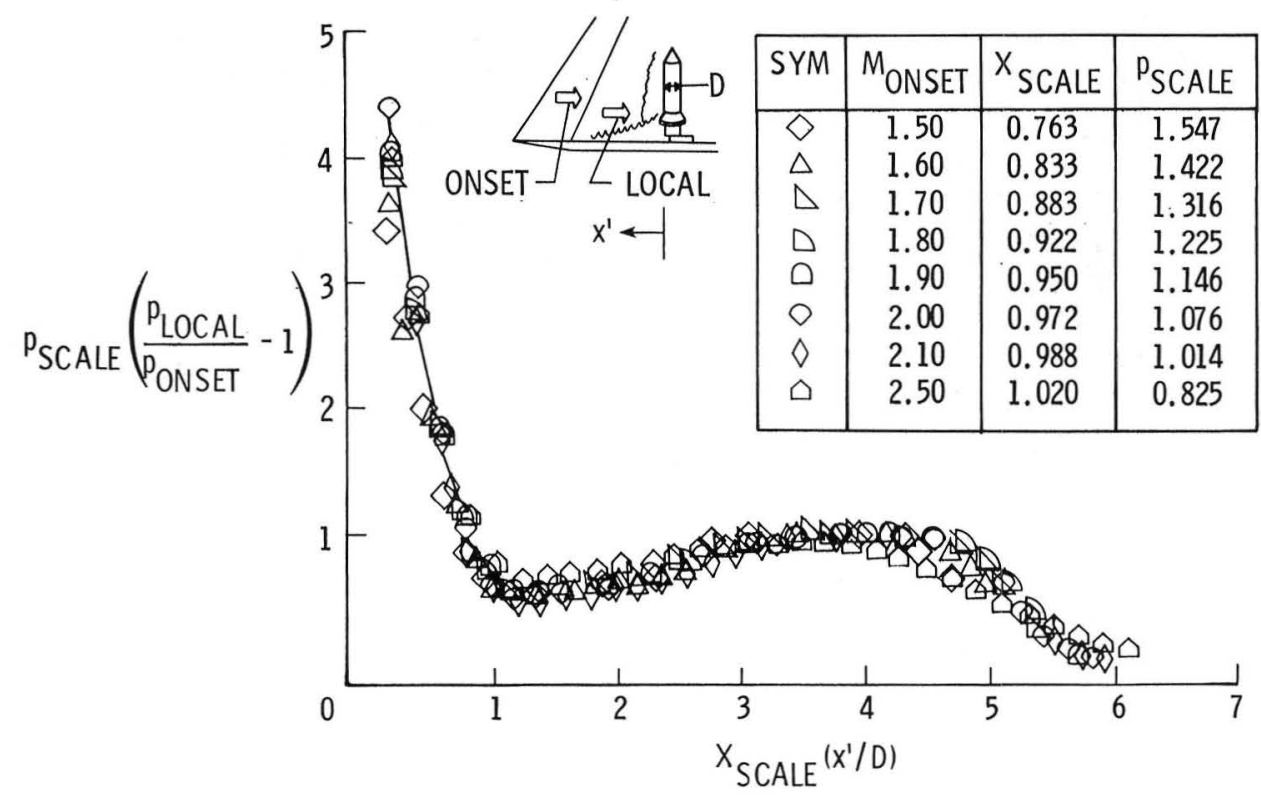

Figure 9.- Variation of nondimensional pressure with nondimensional distance ahead of bipod for a range of Mach numbers from 1.5 to 2.5 .

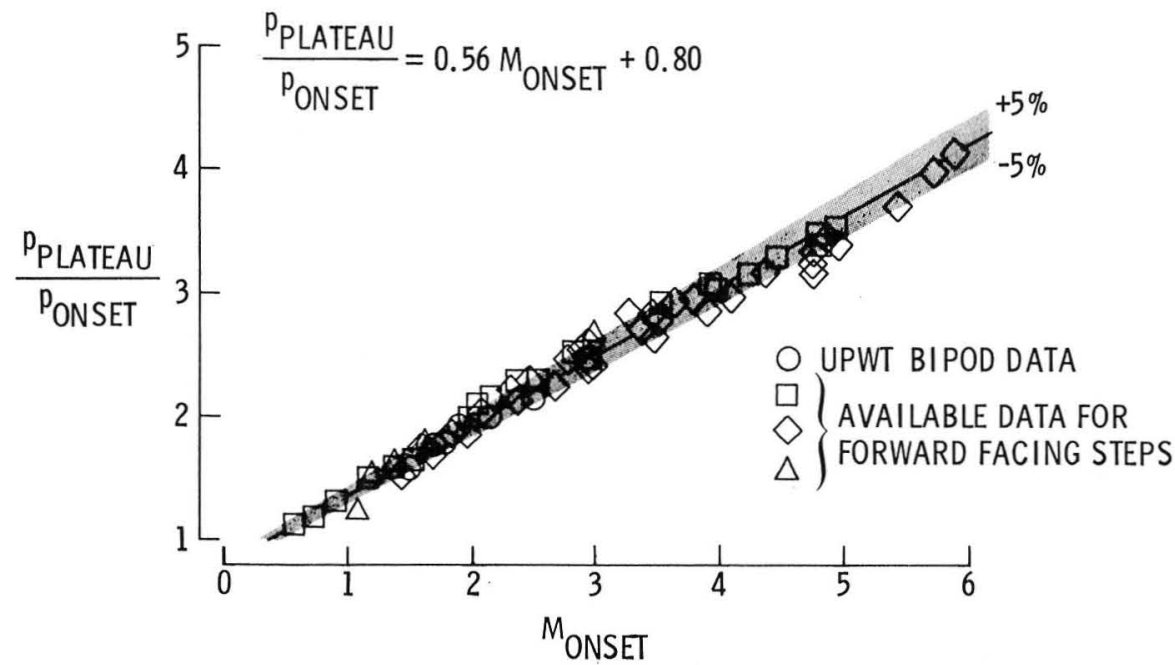

Figure 10.- Variation of PPLATEAU to PONSET ratio with $\mathrm{M}_{\mathrm{ONSET}}$ including comparison of empirical expression with experimental data. 


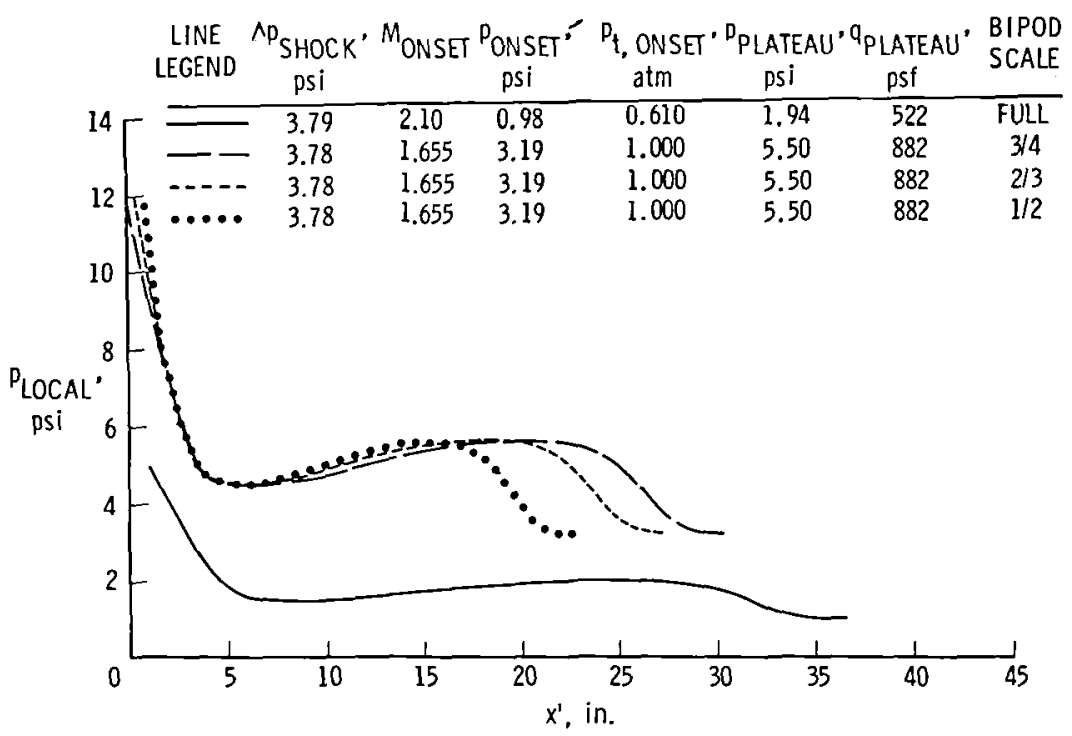

Figure 11.- Comparison of equal bow-shock-strength pressure distributions ahead of bipod, including full-scale bipod at fliqht conditions and several subscale bipods at maximum Mach number expected on 8-ft TPT.

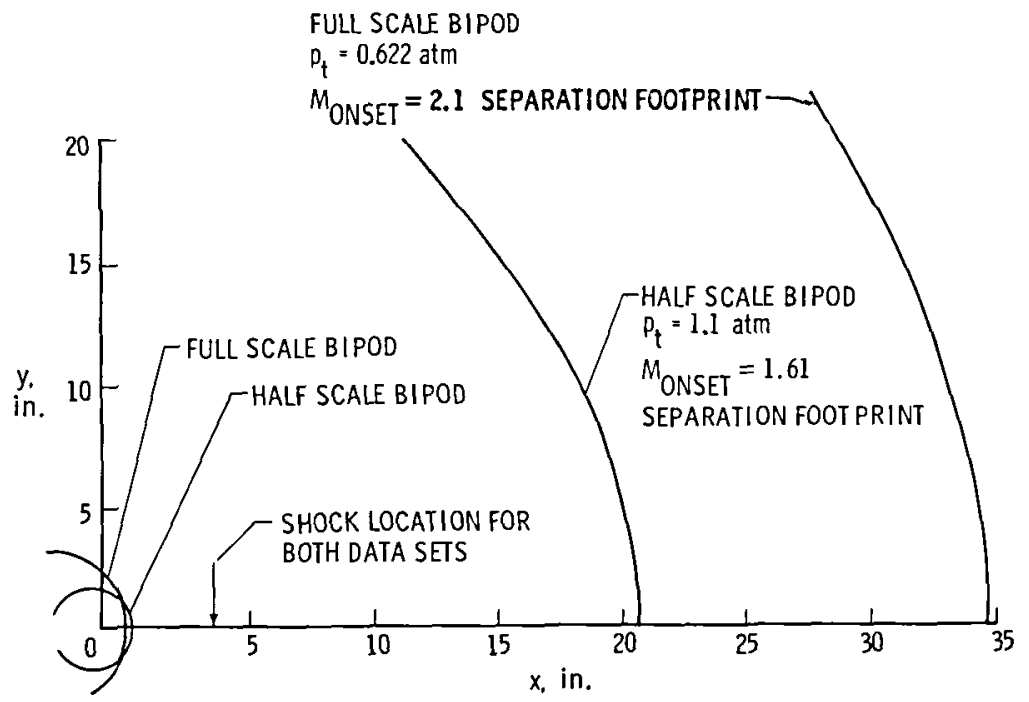

Figure 12.- Separated-flow boundaries ahead of full-scale and half-scale bipods at onset Mach numbers of 2.1 and 1.61 , respectively. 


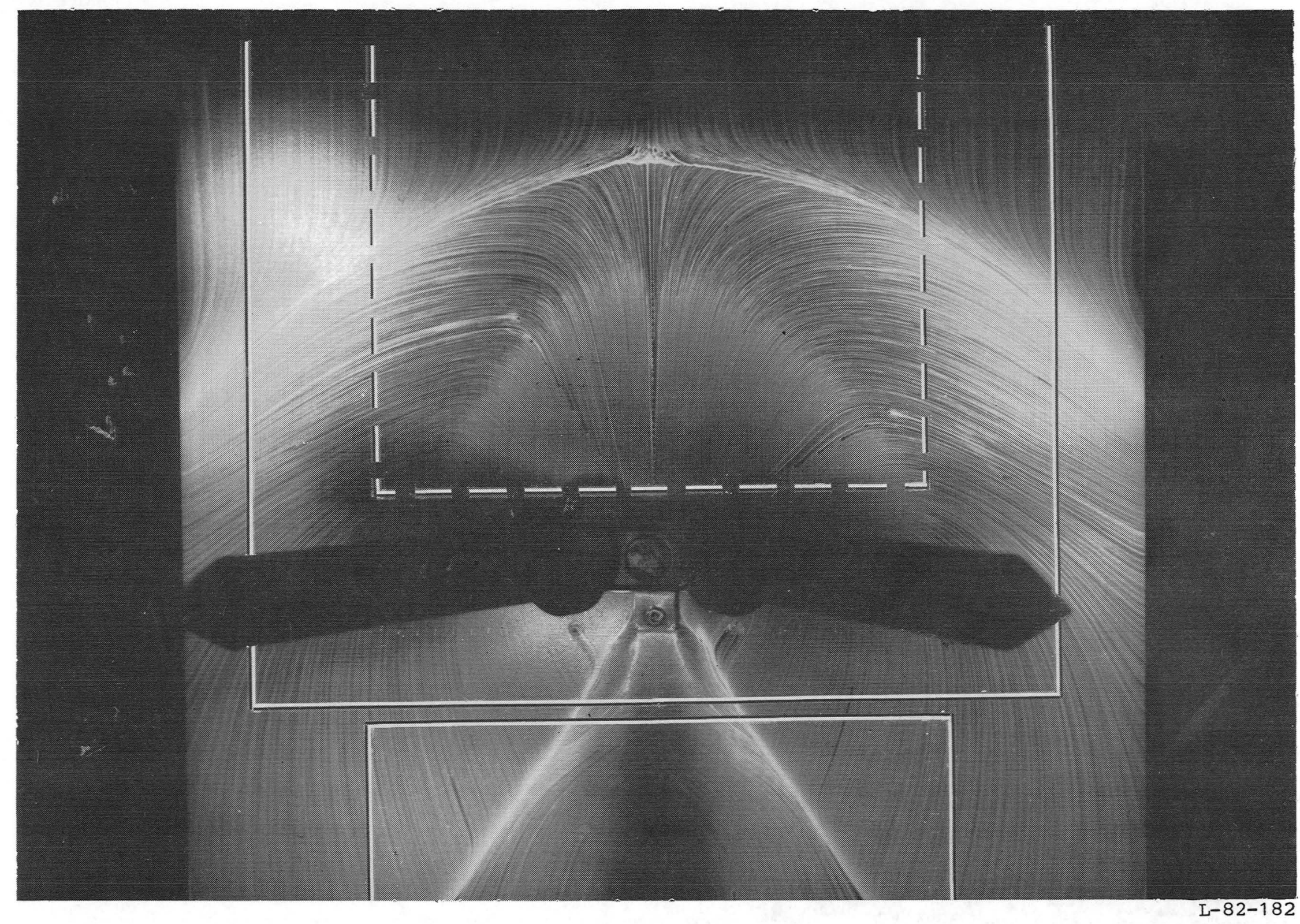

Figure 13.- Oil-flow photographs showing surface streamlines ahead of and behind bipod for three Mach numbers. 


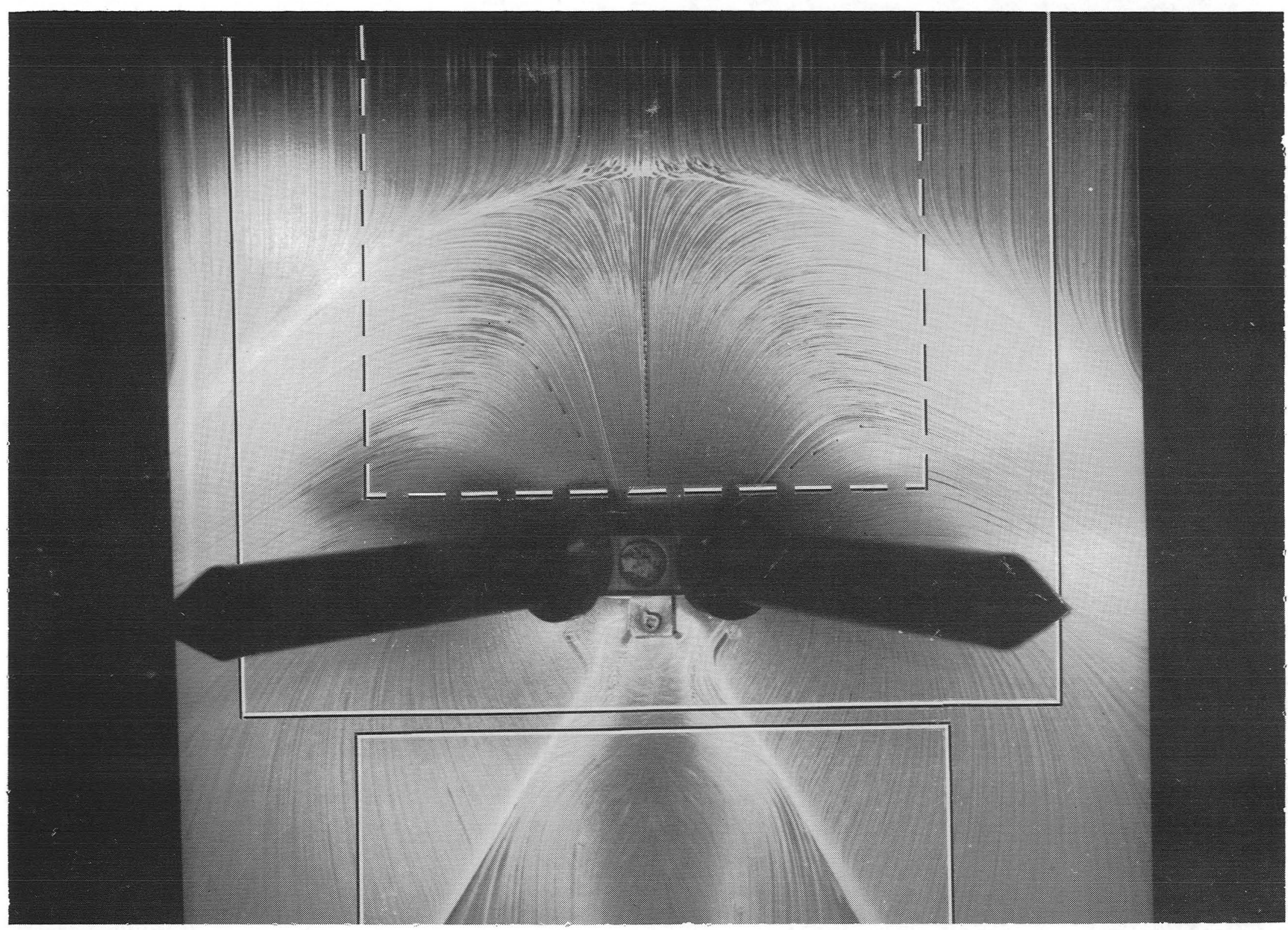

(b) $\mathrm{M}_{\text {ONSET }}=1.8$.

Figure 13.- Continued. 


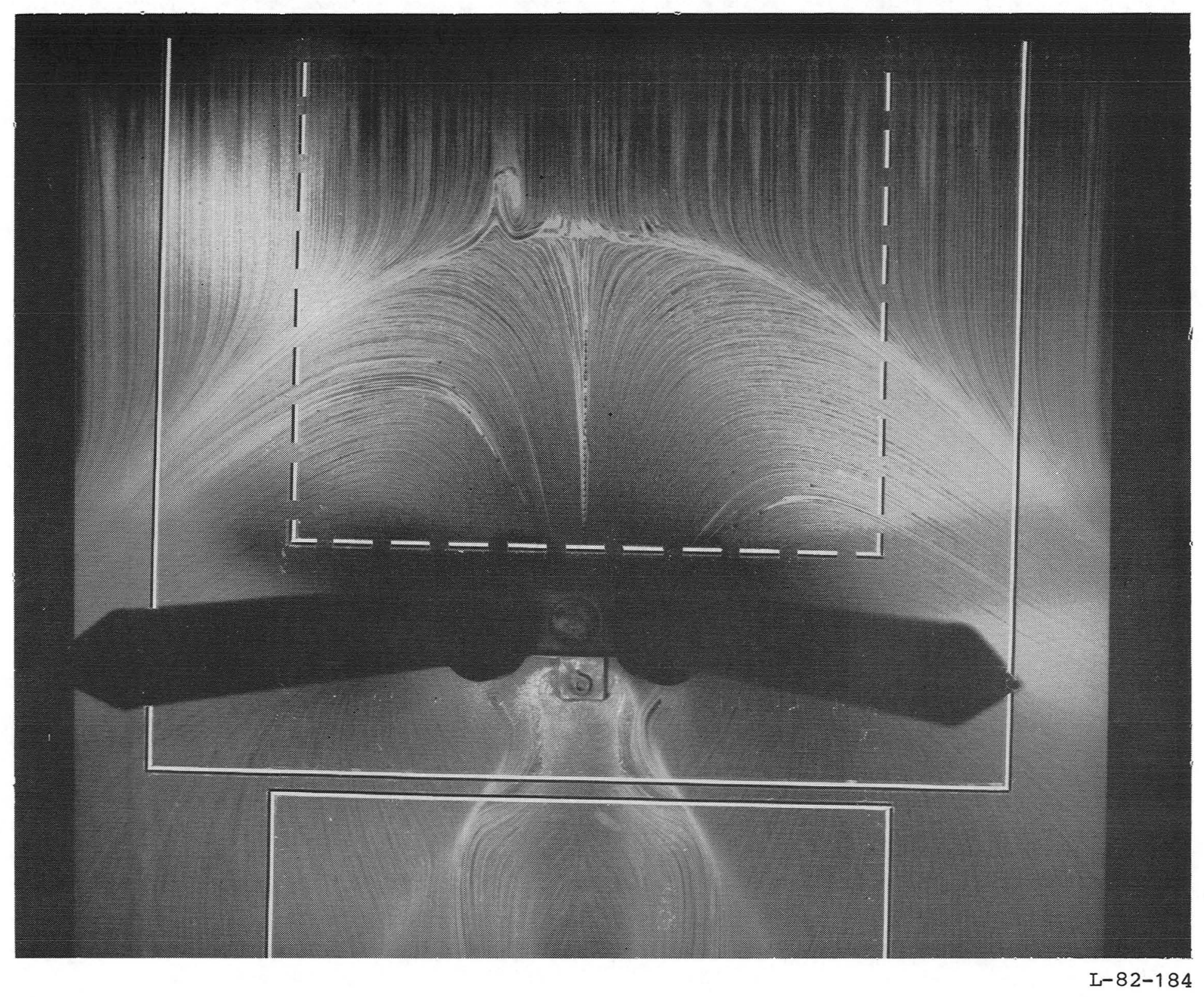

(c) $\mathrm{M}_{\text {ONSET }}=2.1$.

Figure 13.- Concluded. 


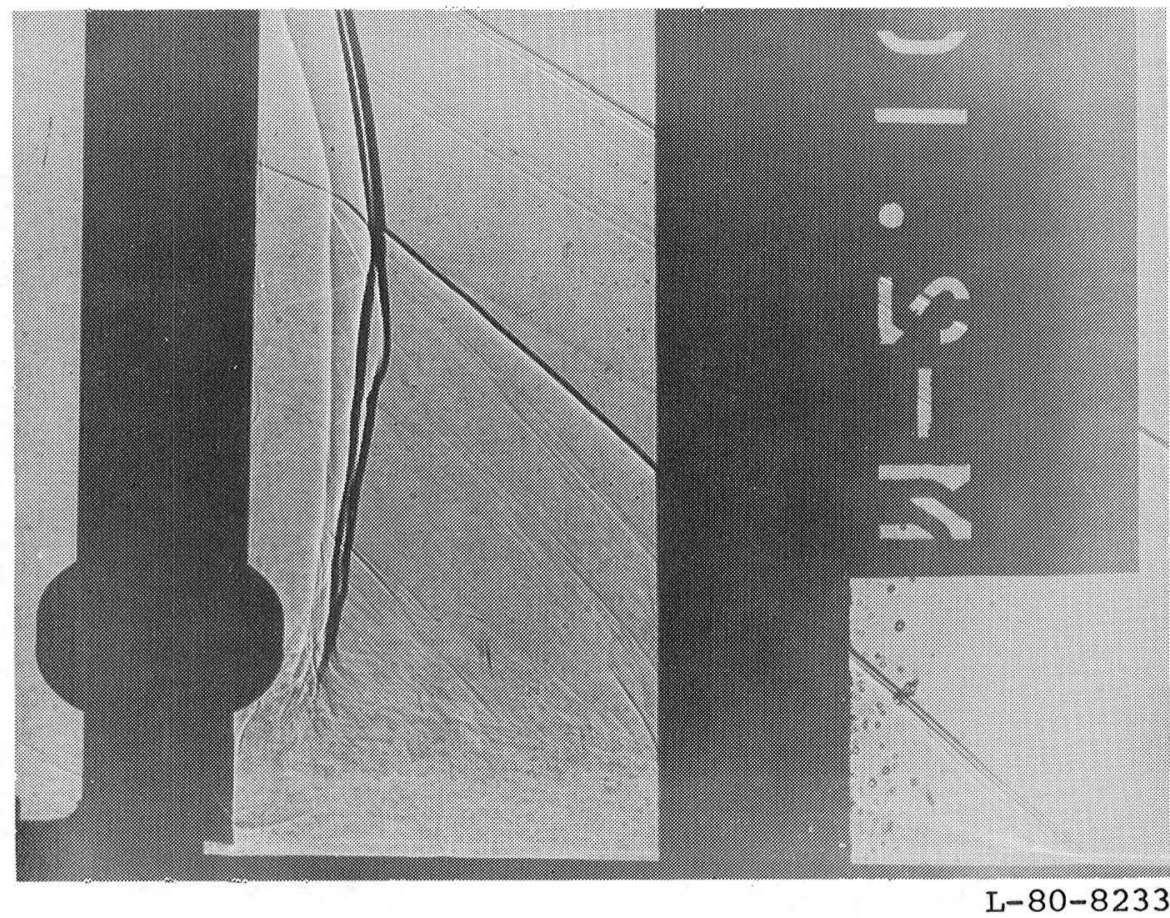

Figure 14.- Shadowgraph of flow ahead of bipod at onset Mach number of 2.1 .

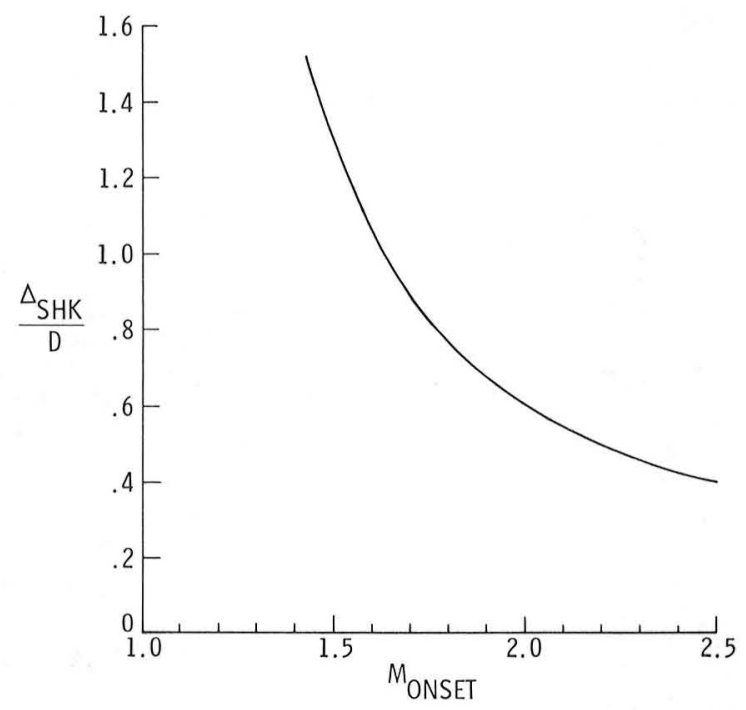

Figure 15.- Variation of detachment distance for bipod bow shock with onset Mach number. 


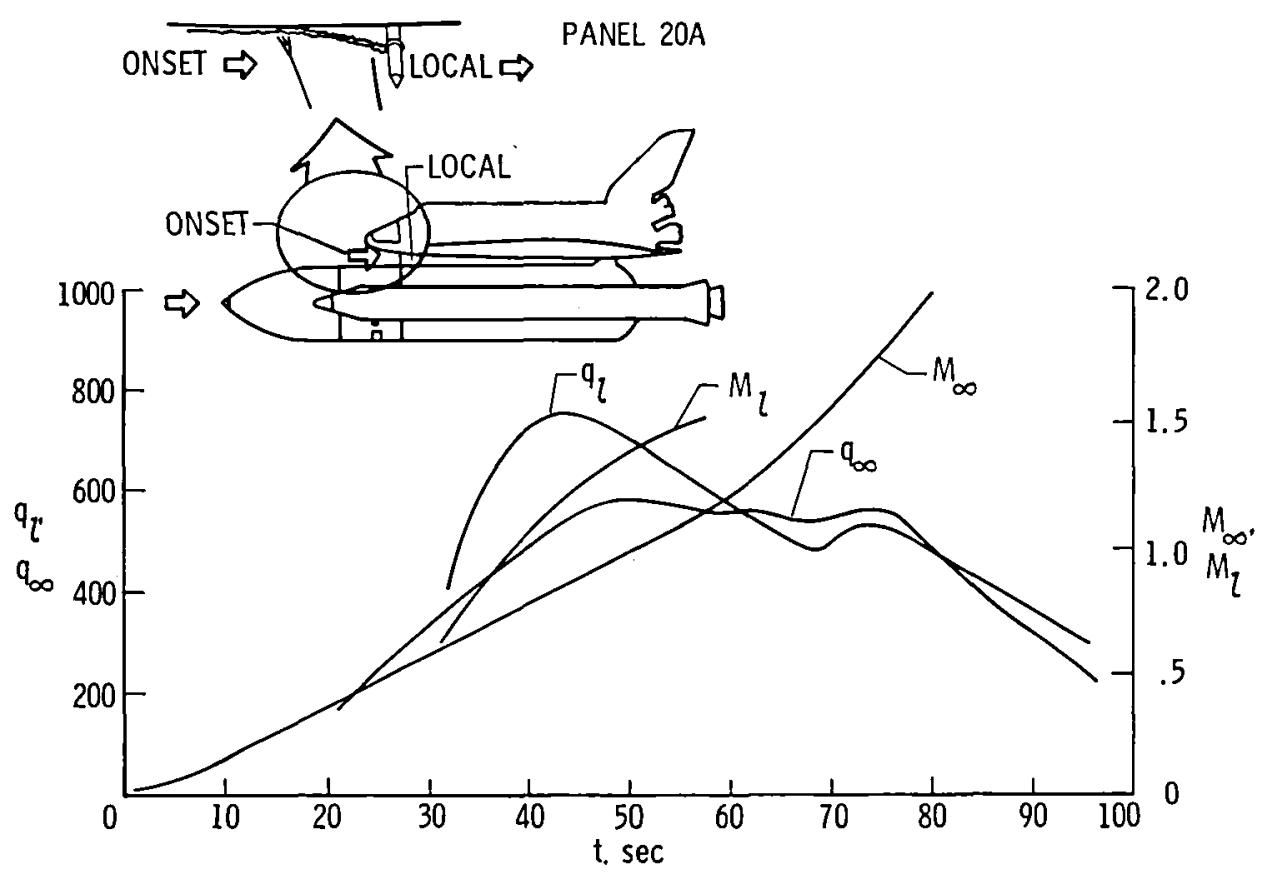

Figure 16.- Variation of free-stream and local values of Mach number and dynamic pressure with time for panel $20 A$ simulation.

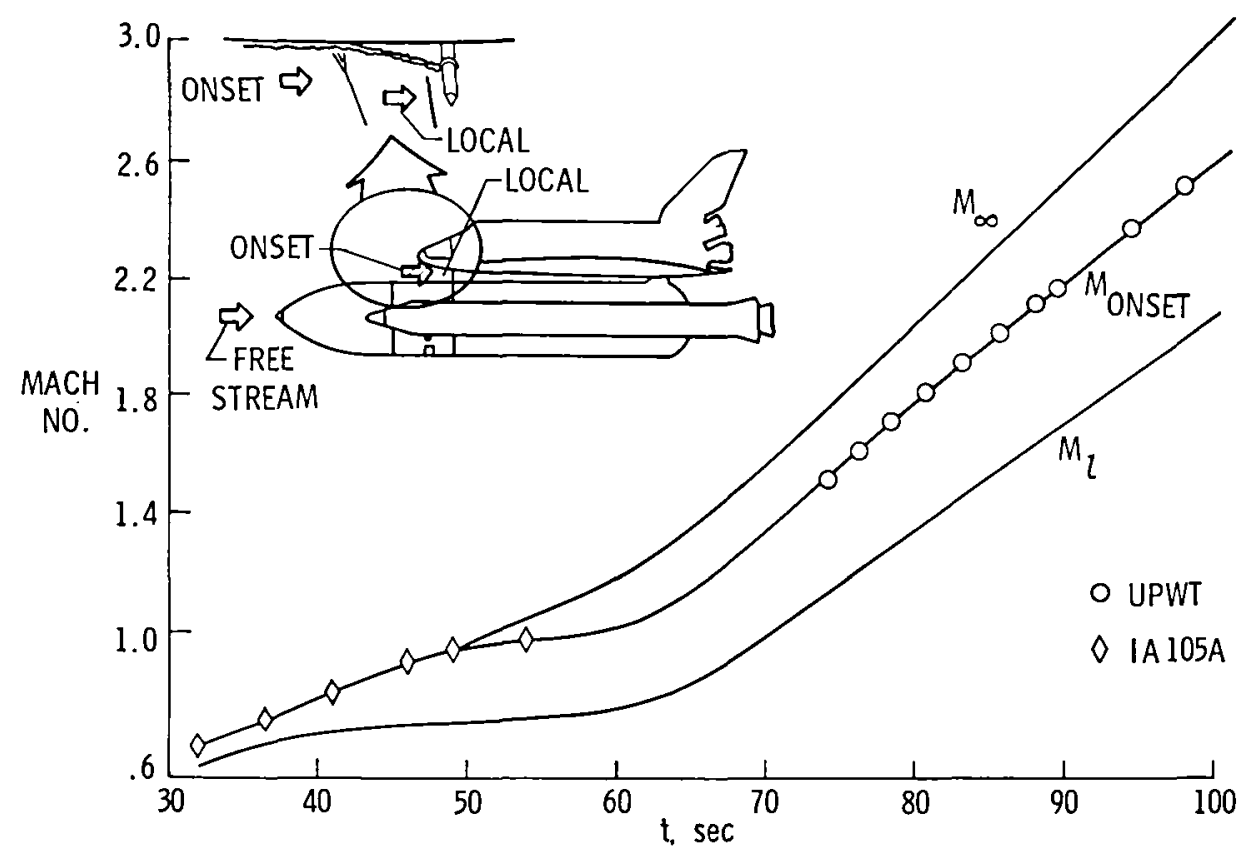

Figure 17.- Variations of free-stream, onset, and local Mach numbers with time for panel $20 \mathrm{C}$ simulation. 


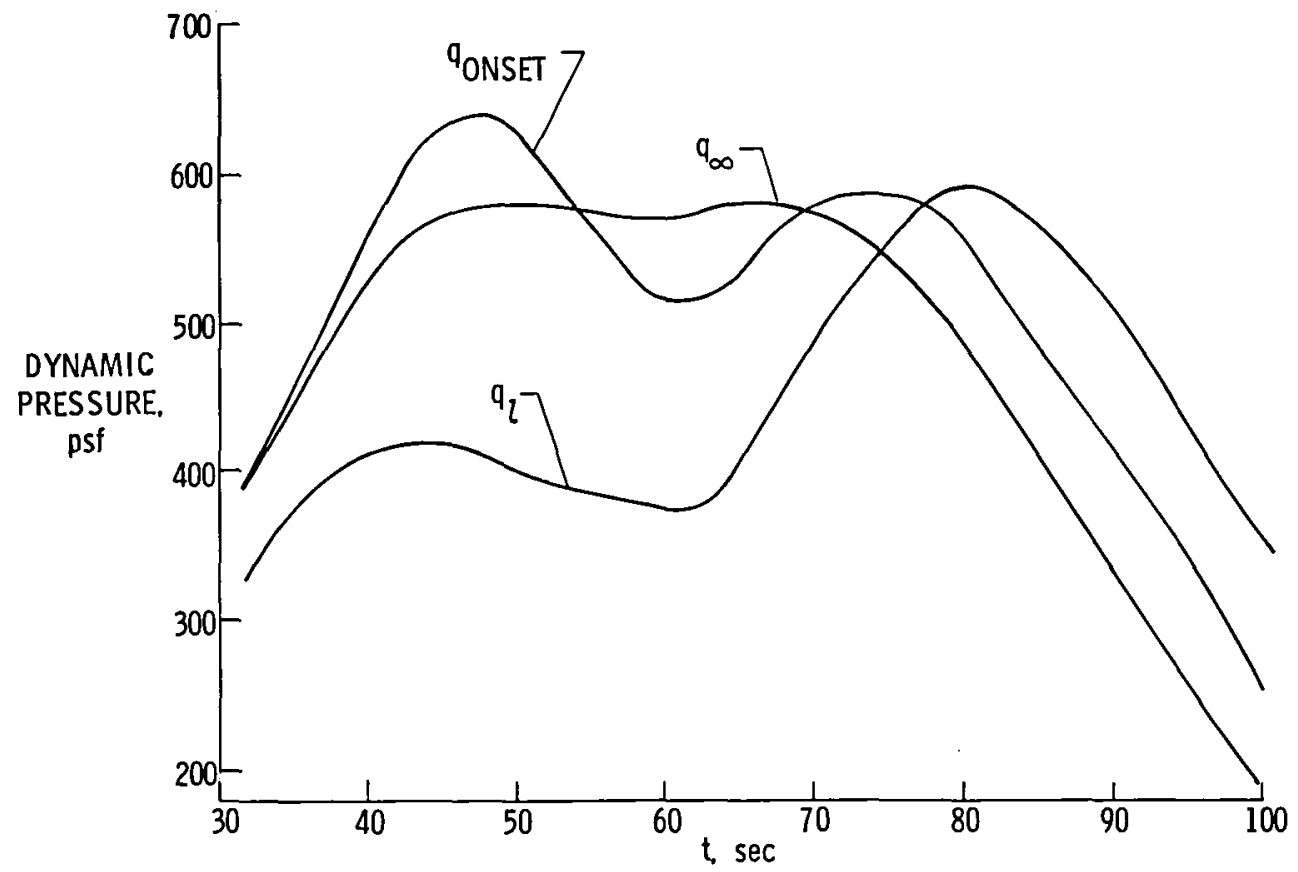

Figure 18.- Variation of free-stream, onset, and local dynamic pressures with time for panel $20 \mathrm{C}$ simulation.

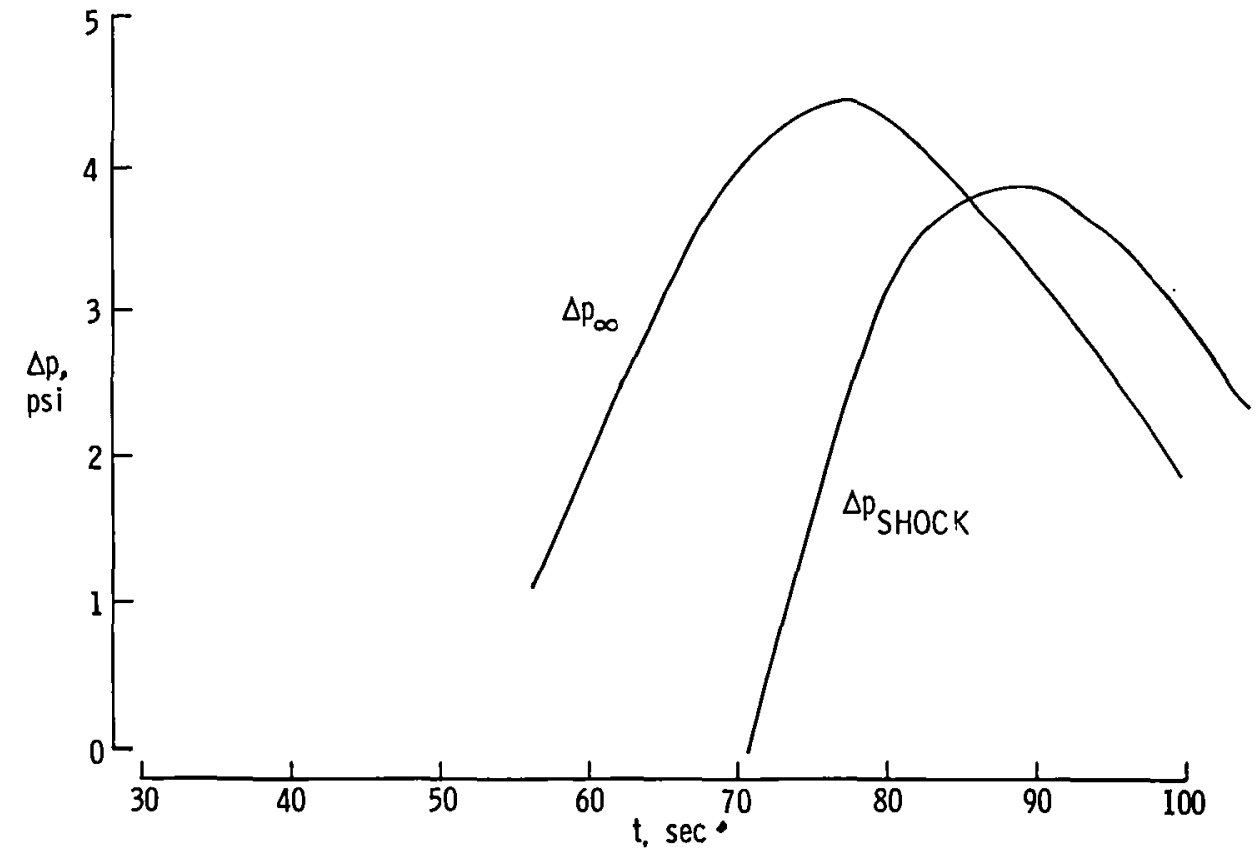

Figure 19.- Variation of pressure jump across bipod bow shock with time compared to that for a normal shock with free-stream onset conditions. 


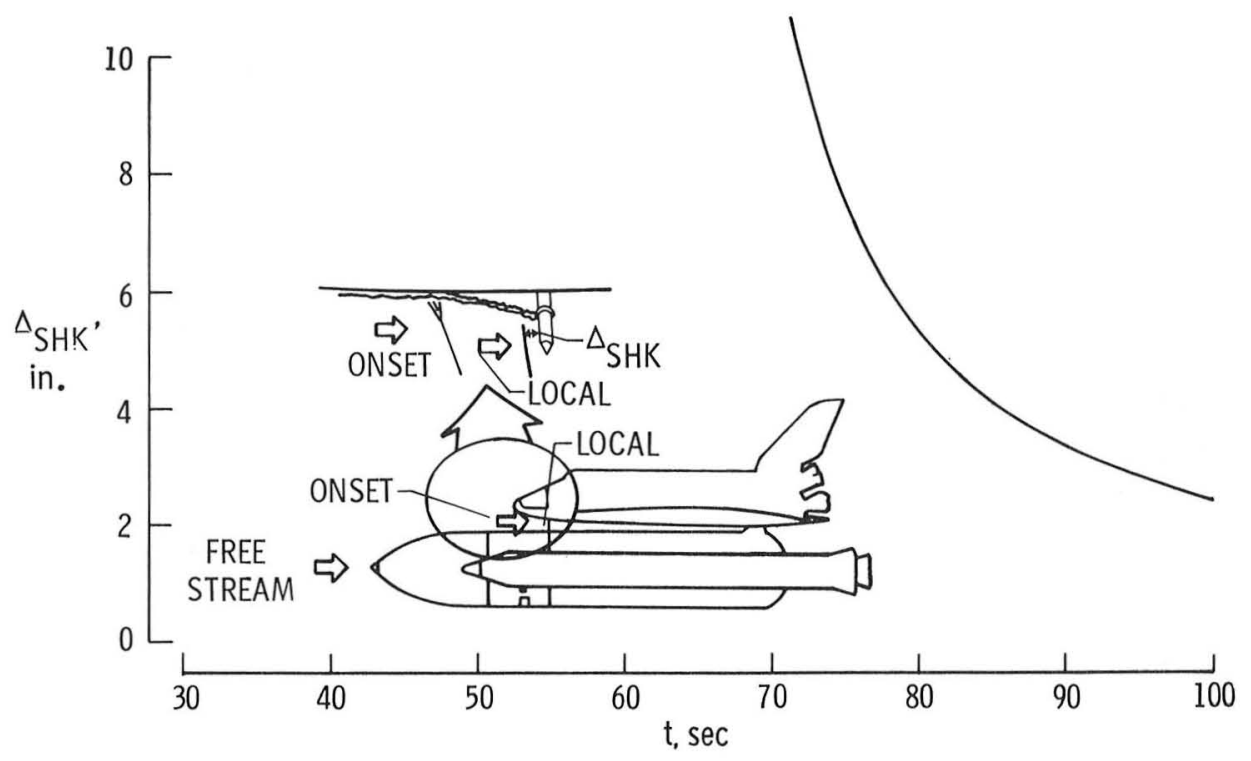

Figure 20.- Variation of bow-shock detachment distance with time for STS-1.

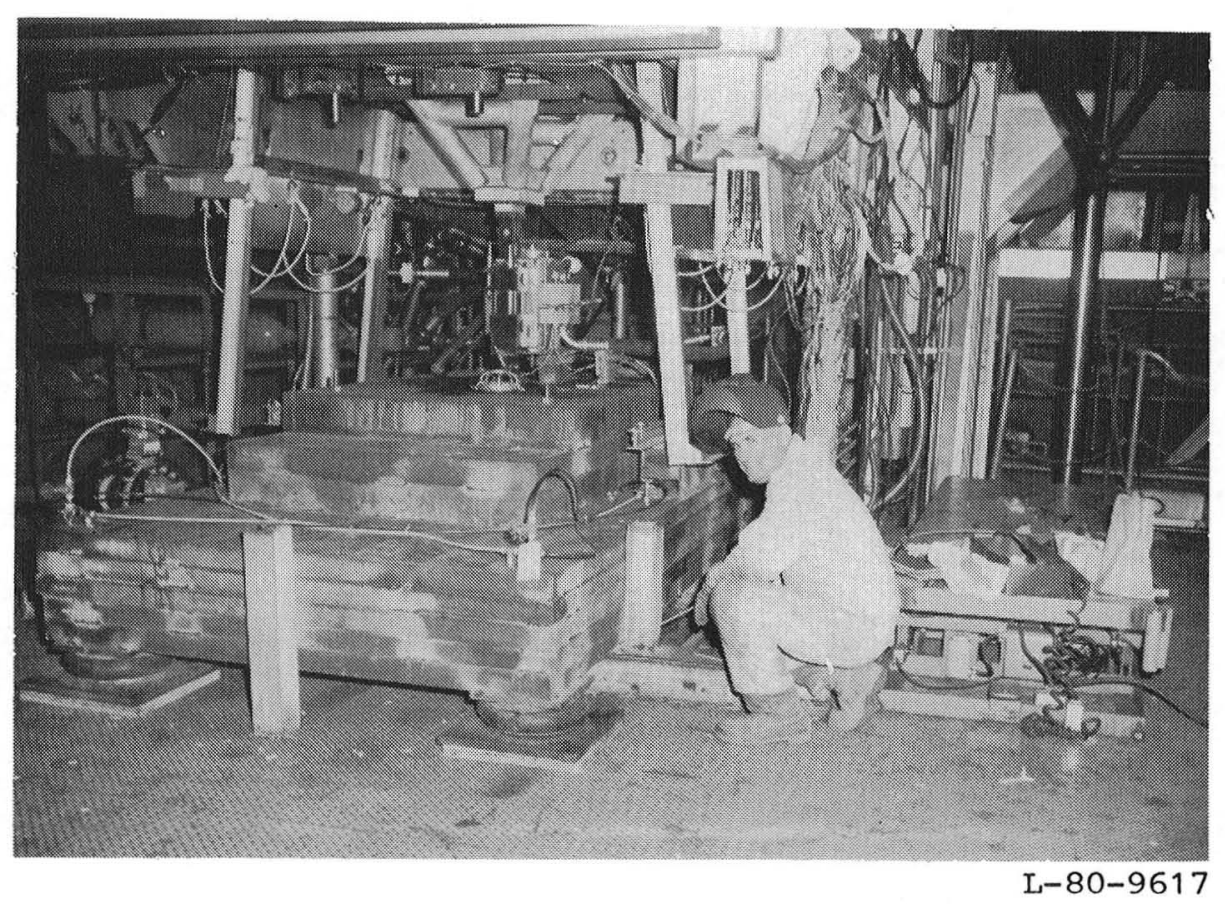

Figure 21.- Hydraulic shaker mounted below test section of 8-ft TPT on 60 000-pound backstop. 


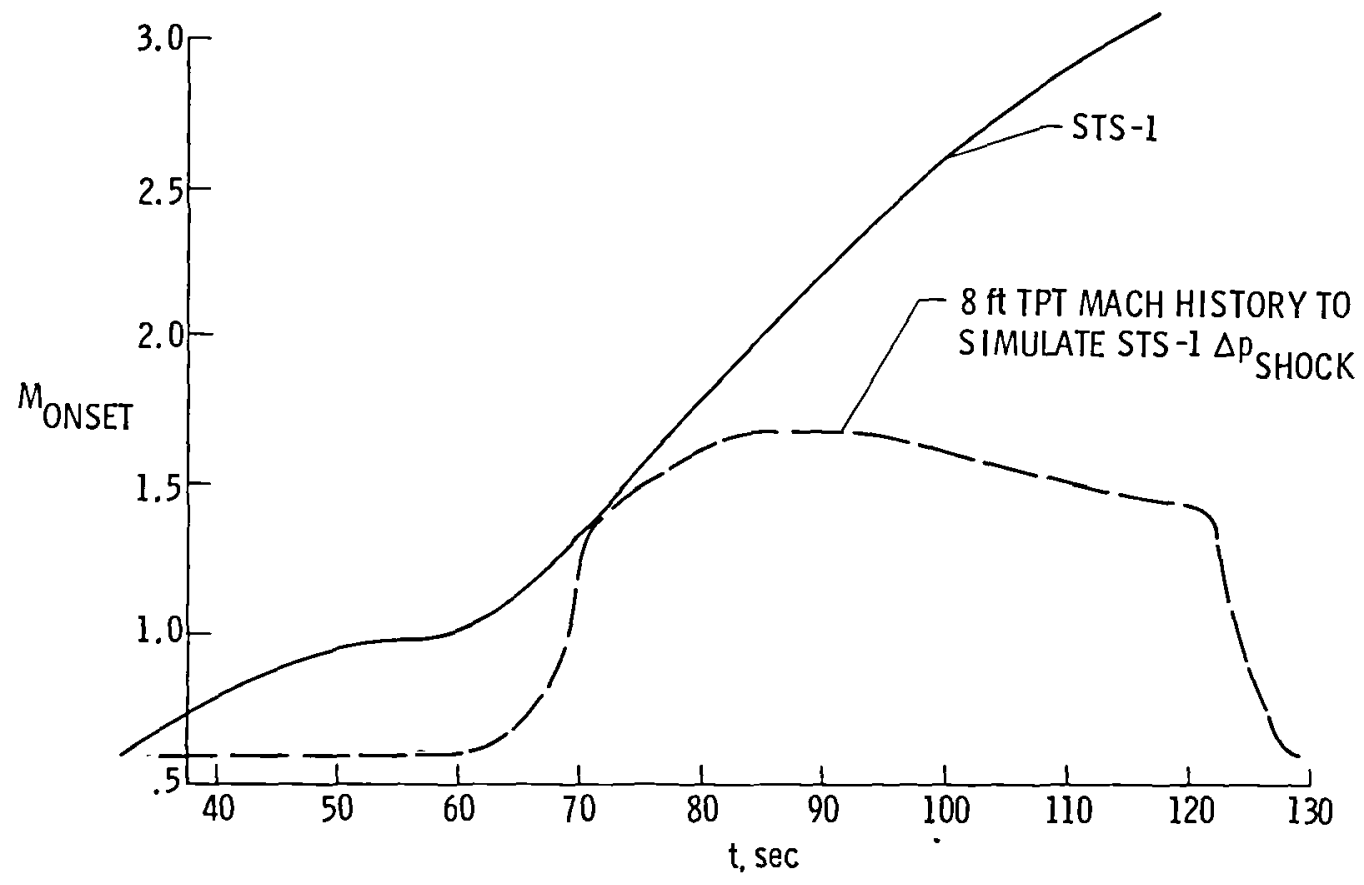

Figure 22.- Time variation of STS-1 onset Mach number compared with that required in 8-ft TPT simulation to match STS-1 bow-shock pressure jumps.

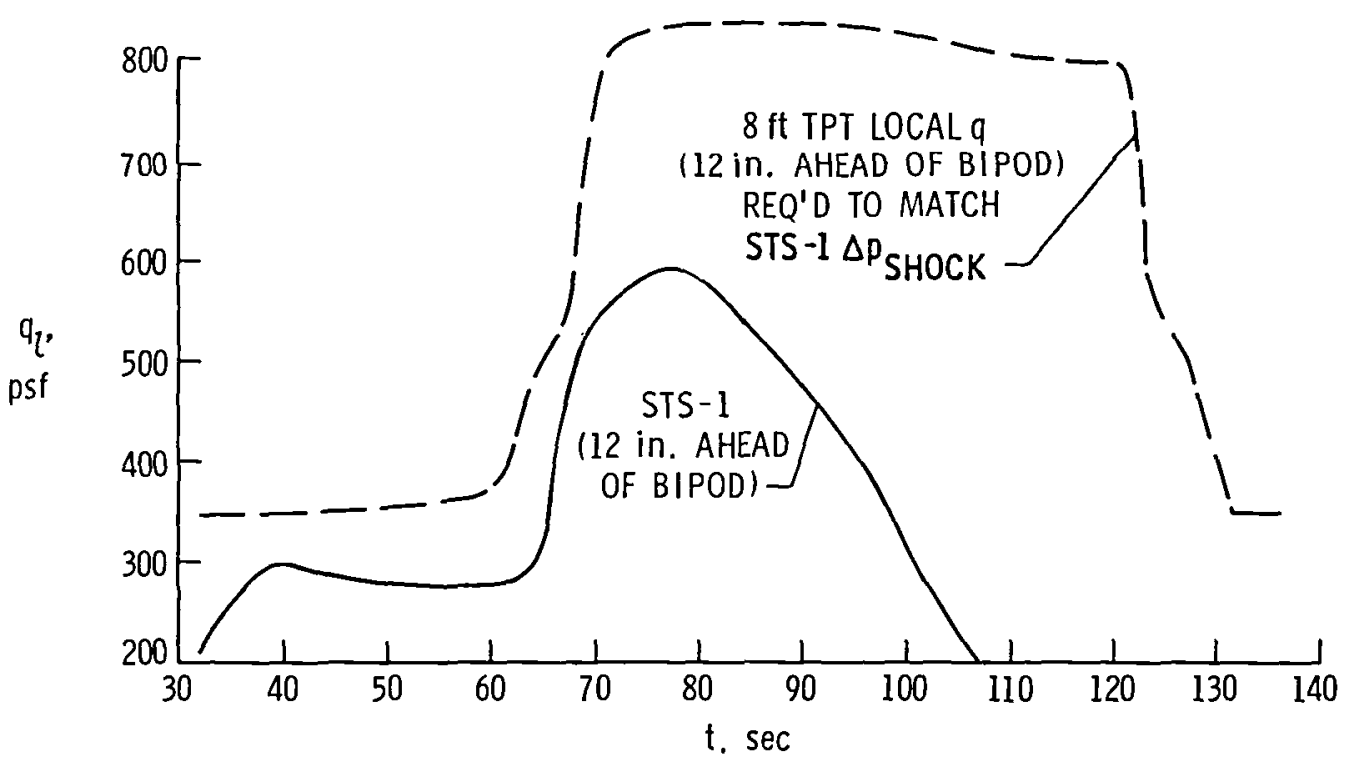

Figure 23.- Comparison of time variations of dynamic pressure at a point 12 in. ahead of full-scale bipod for STS-1 trajectory with that 12 in. ahead of half-scale bipod in $8-f t$ TPT when simulating STS-1 $\Delta \mathrm{p}_{\text {SHOCK }}$ time history. 


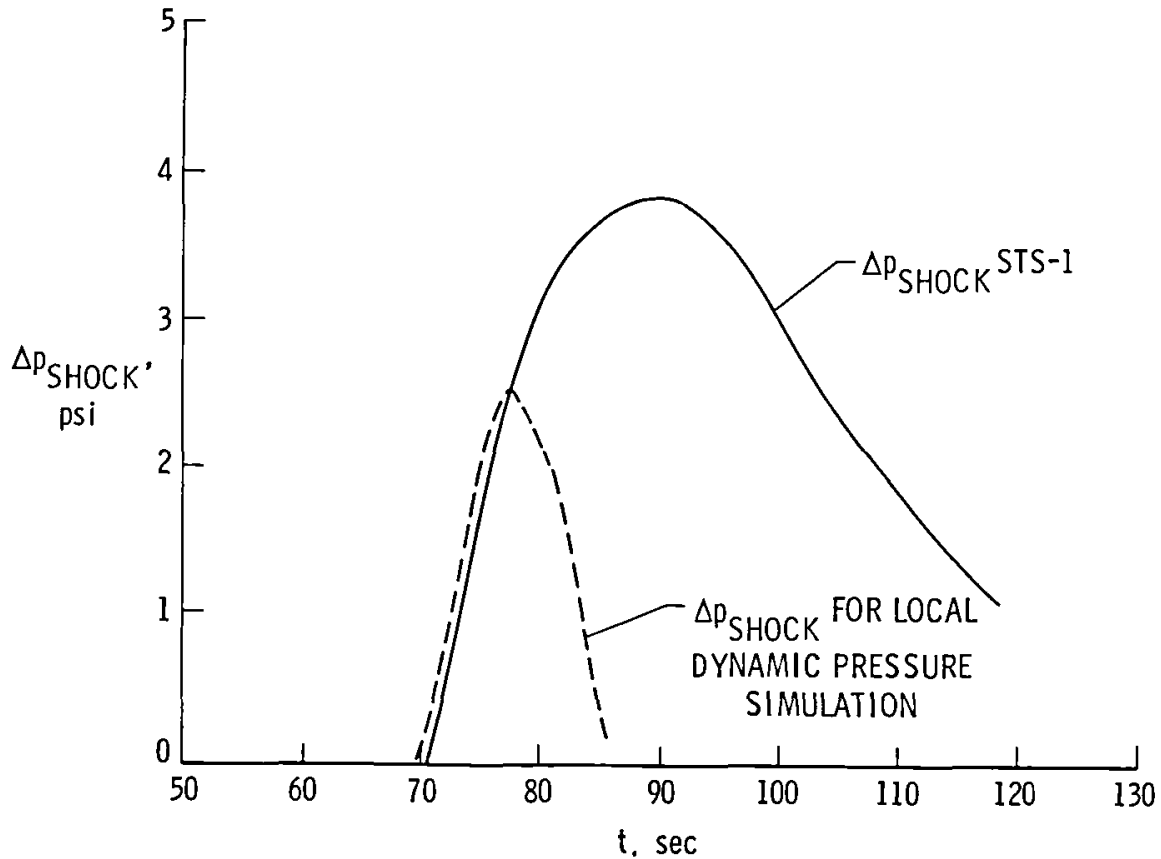

Figure 24.- Shock-pressure jump versus time for STS-1 trajectory and for 8-ft TPT when simulating time variation of local dynamic pressure $q_{2}$.

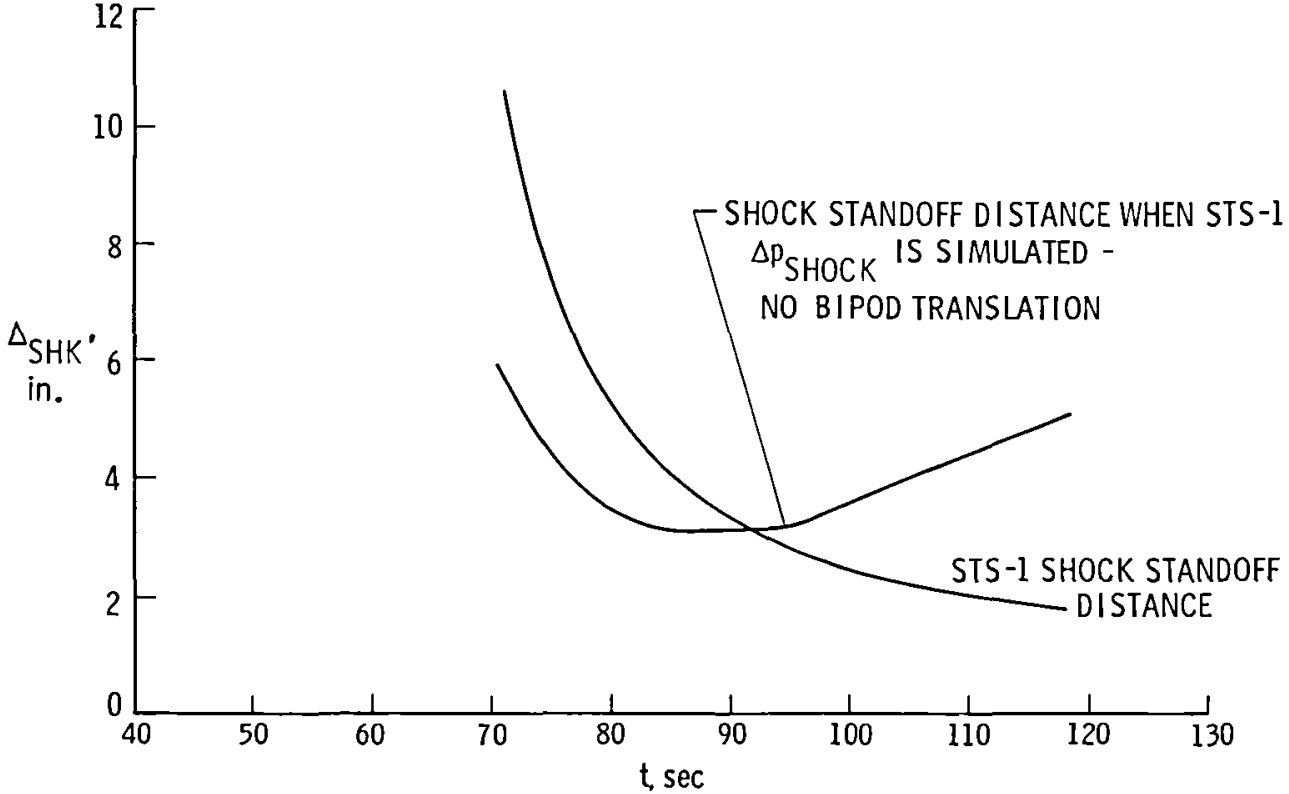

Figure 25.- Variation of shock standoff distance for STS-1 trajectory with time compared with that for fixed halfscale bipod in 8-ft TPT during simulation of STS-1 $\triangle \mathrm{p}_{\text {SHOCK }}$ time variations. 


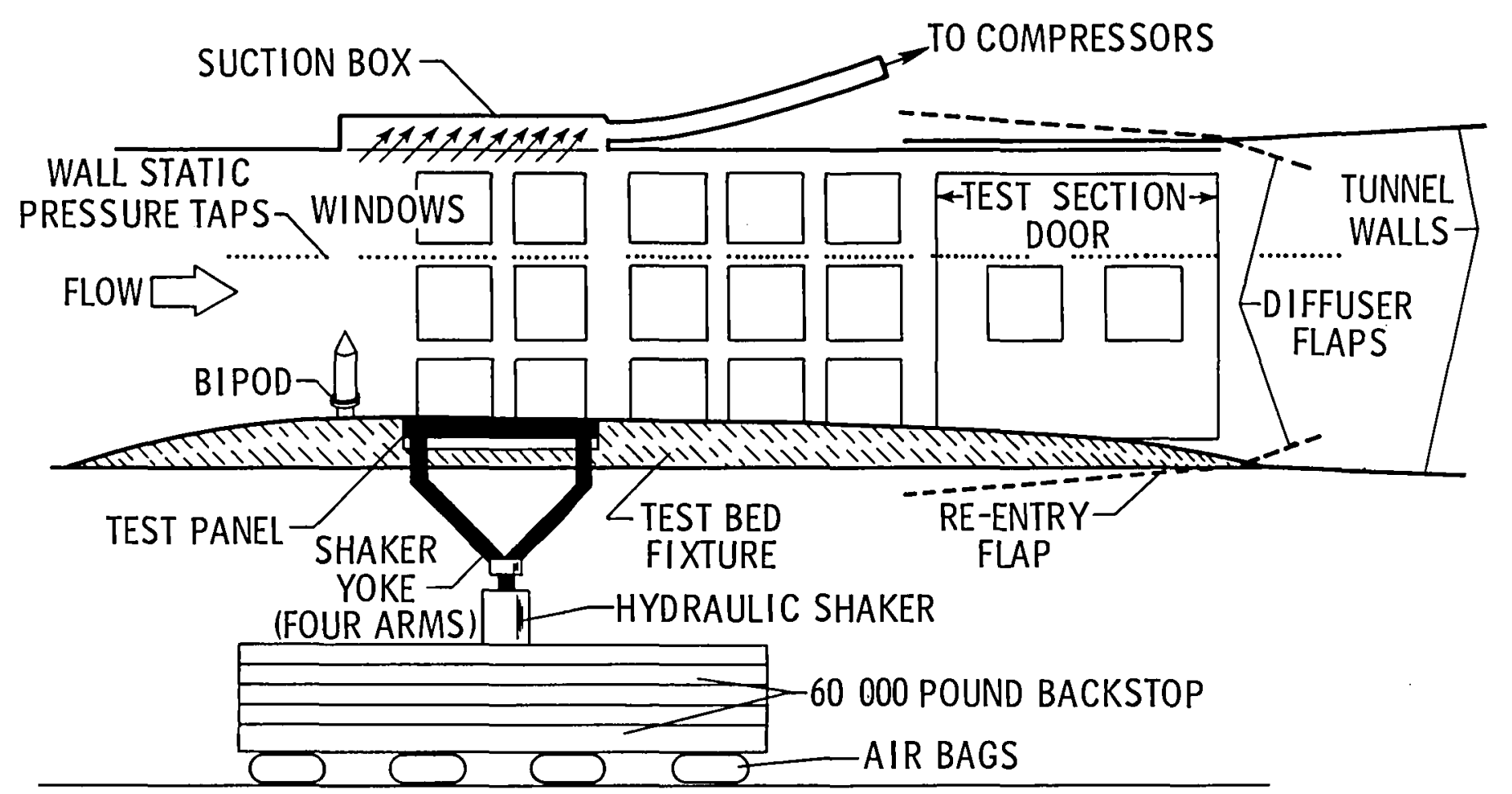

Figure 26.- Sketch of 8-ft TPT test section with test setup for panel 20A. 


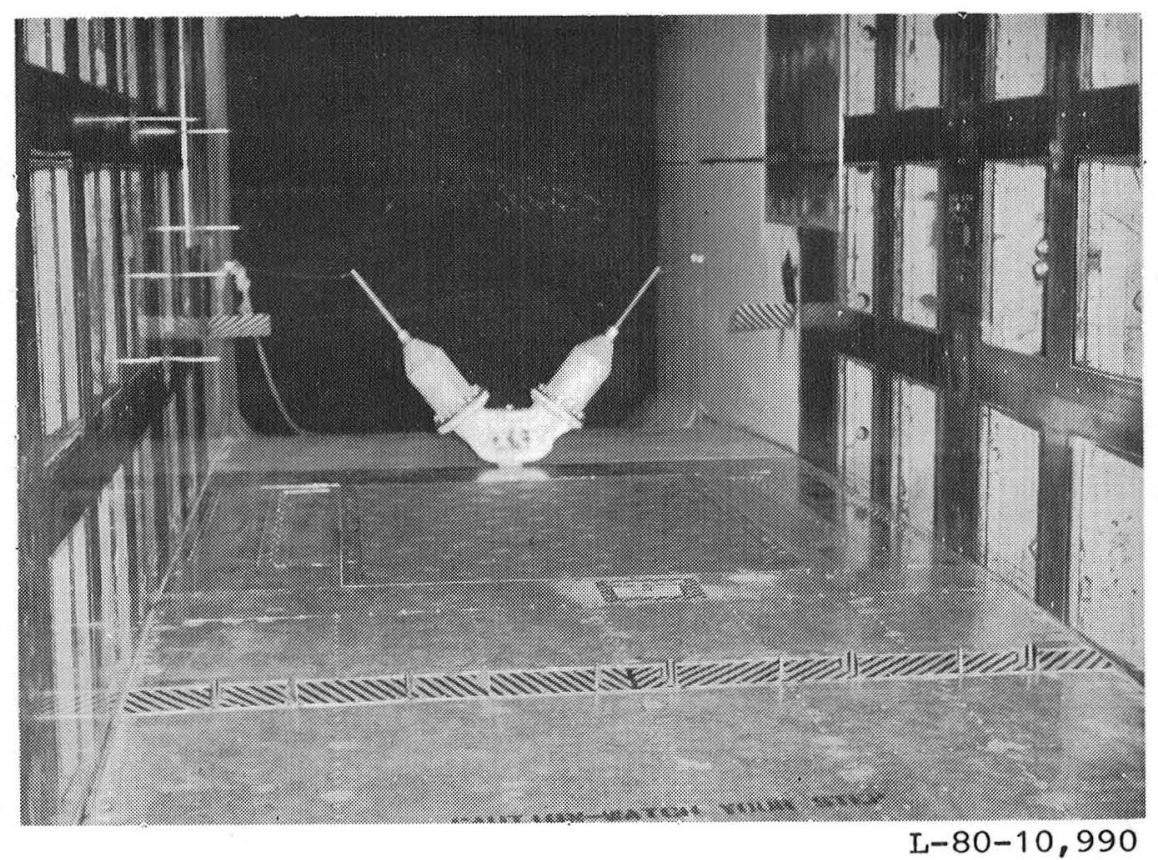

Figure 27.- Photograph of 8-ft TPT test section with panel 20A installed in false floor.

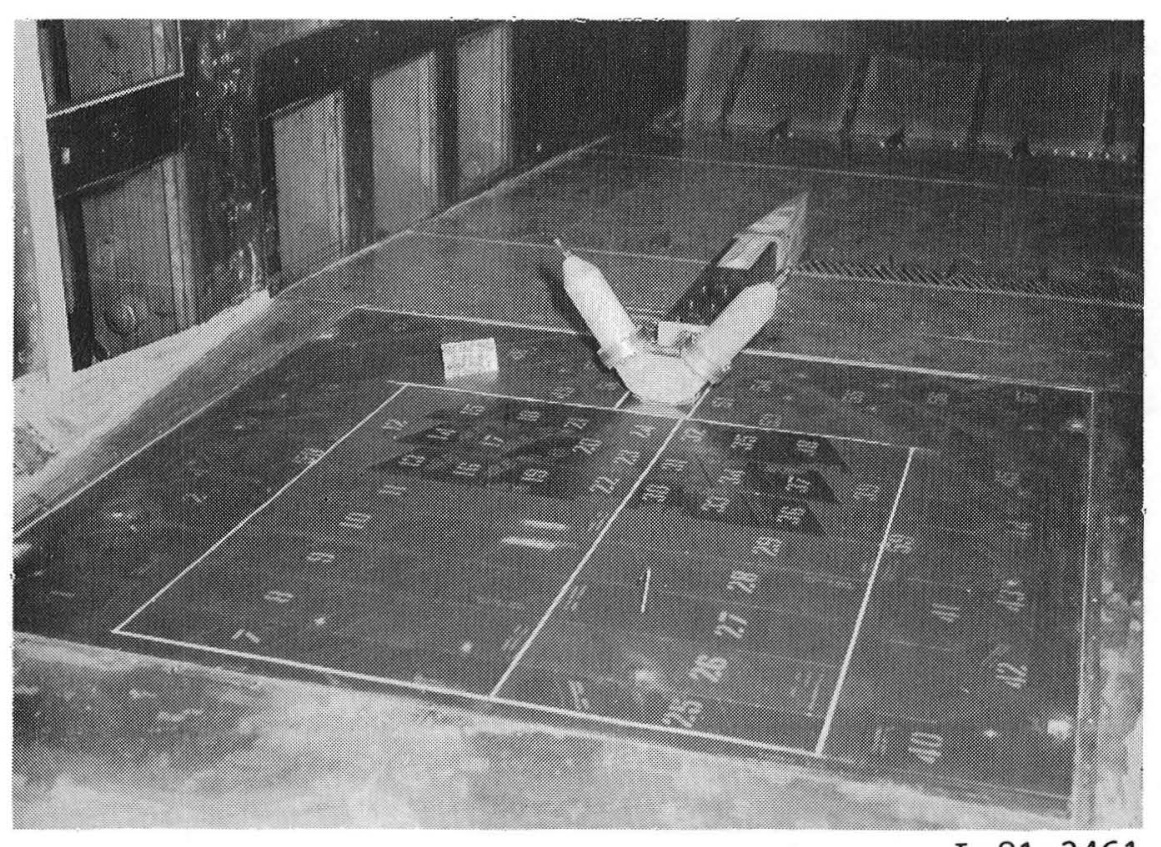

Figure 28.- Photograph of panel 20C installed in false floor in 8-ft TPT test section. 


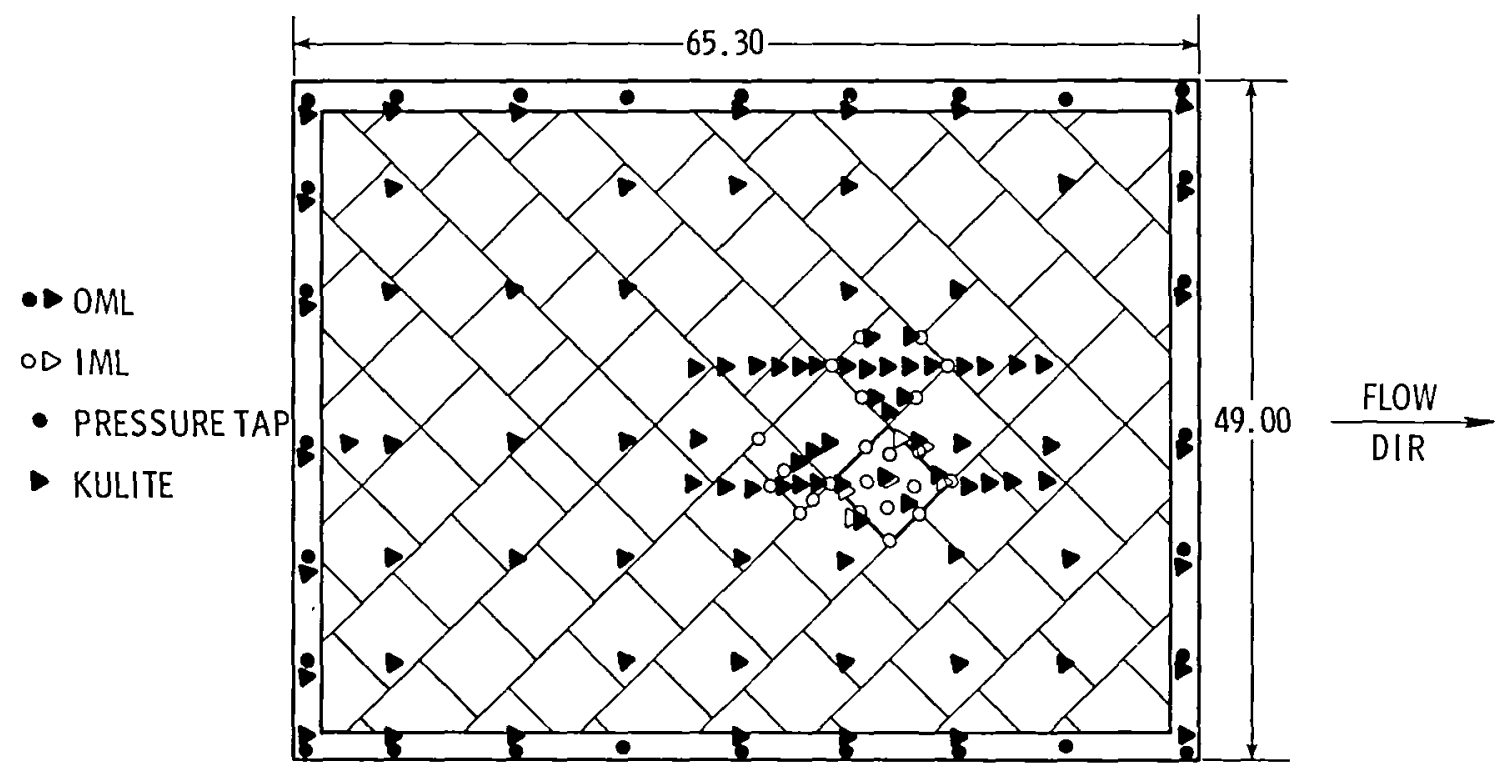

(a) Dynamic- and static-pressure gages located on surface and under tiles of panel 20A. All dimensions are in inches.

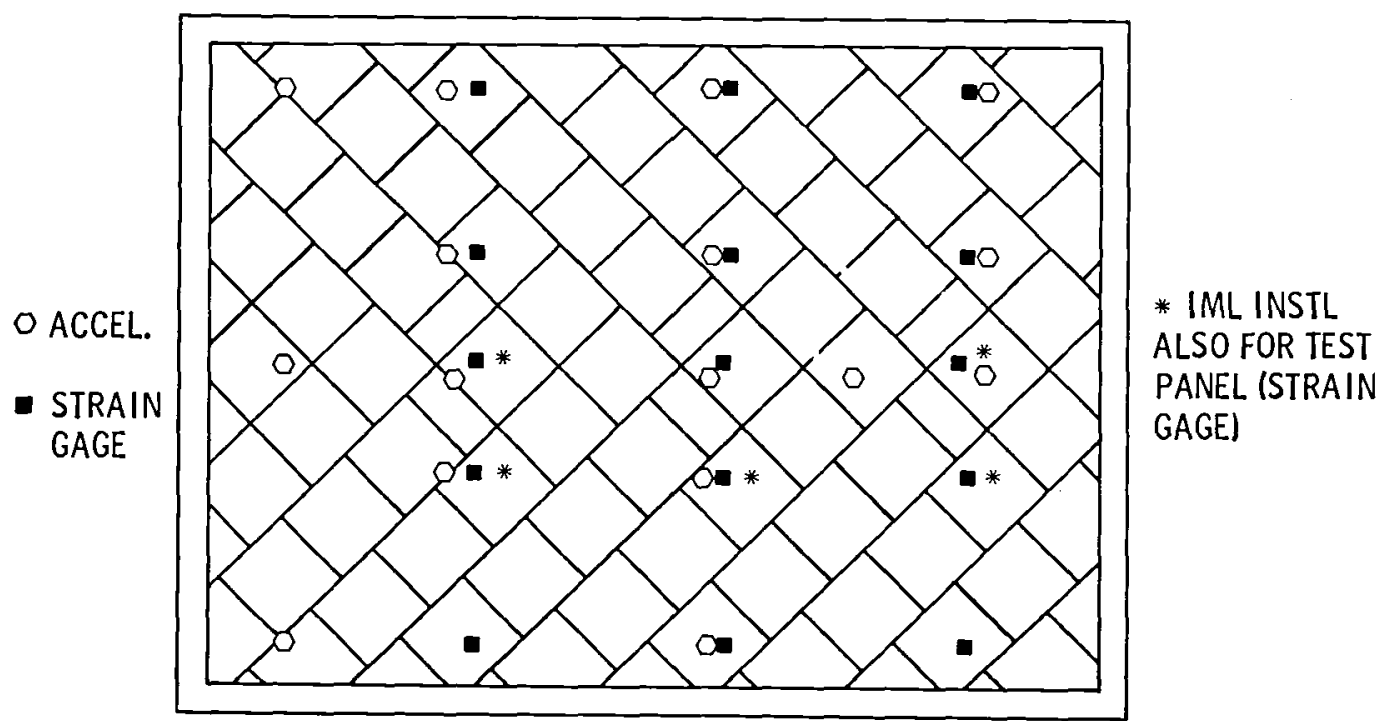

LOCATIONS SHOWN ARE ON FAR SIDE OF PANEL

(b) Accelerometer and strain-gage installation on panel $20 \mathrm{~A}$.

Figure 29.- Sketch of locations and types of instrumentation on calibration panel for panel 20A. 


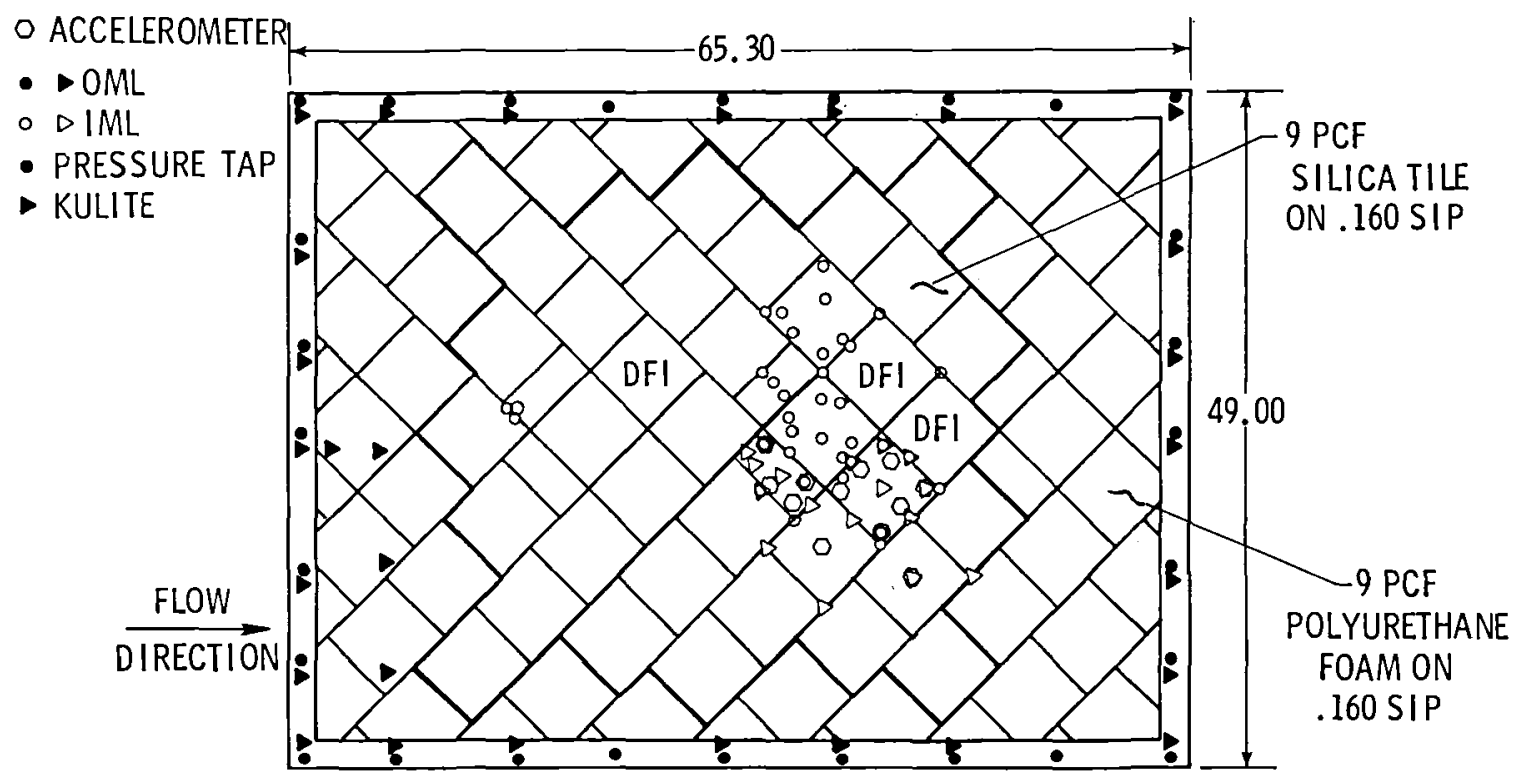

Figure 30.- Sketch showing instrumentation and types of tiles on test panel for configuration 20A. All dimensions are in inches.

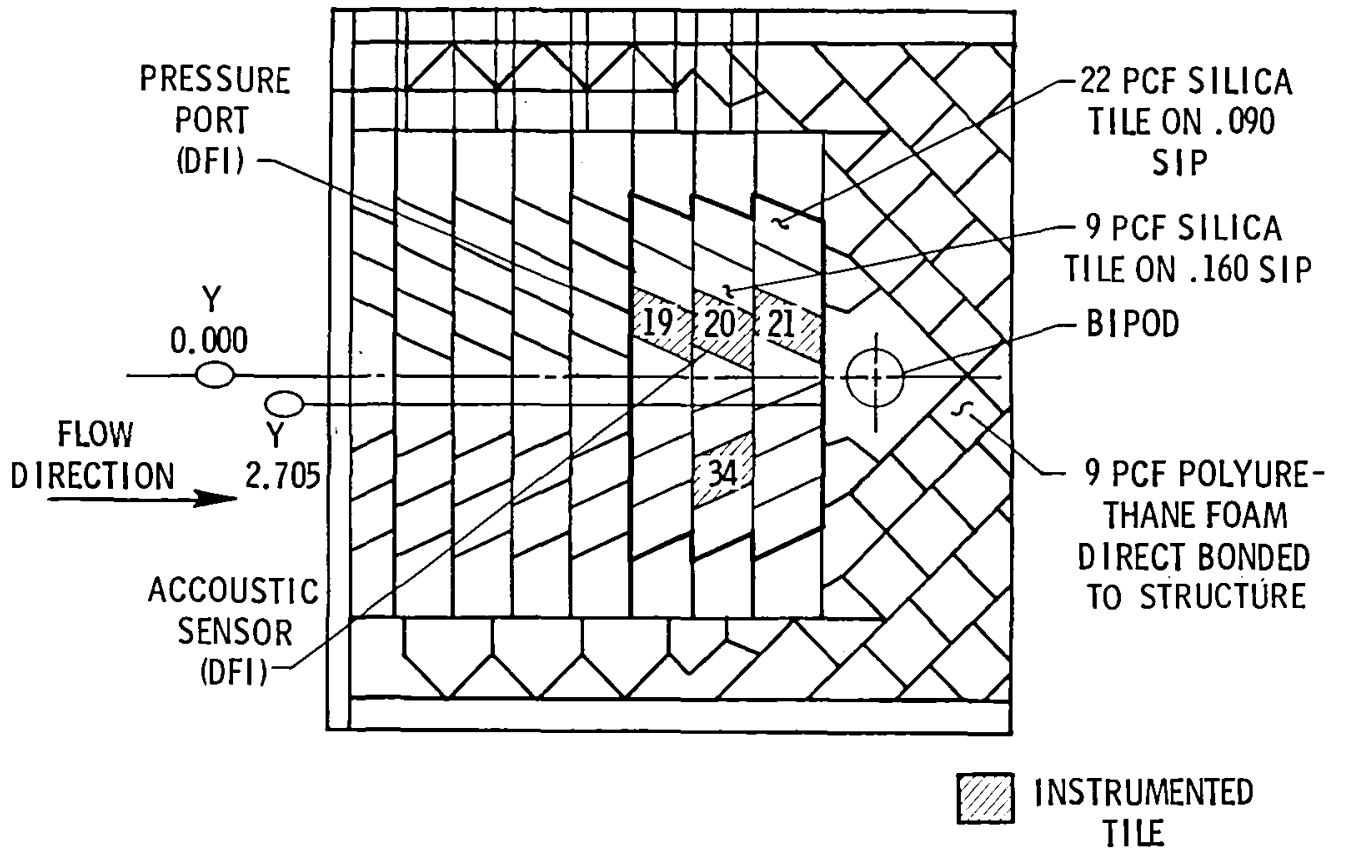

Figure 31.- Sketch showing location of instrumented tiles and types of tiles installed on test panel for configuration $20 \mathrm{C}$. 
SIMULATION PROCEDURE

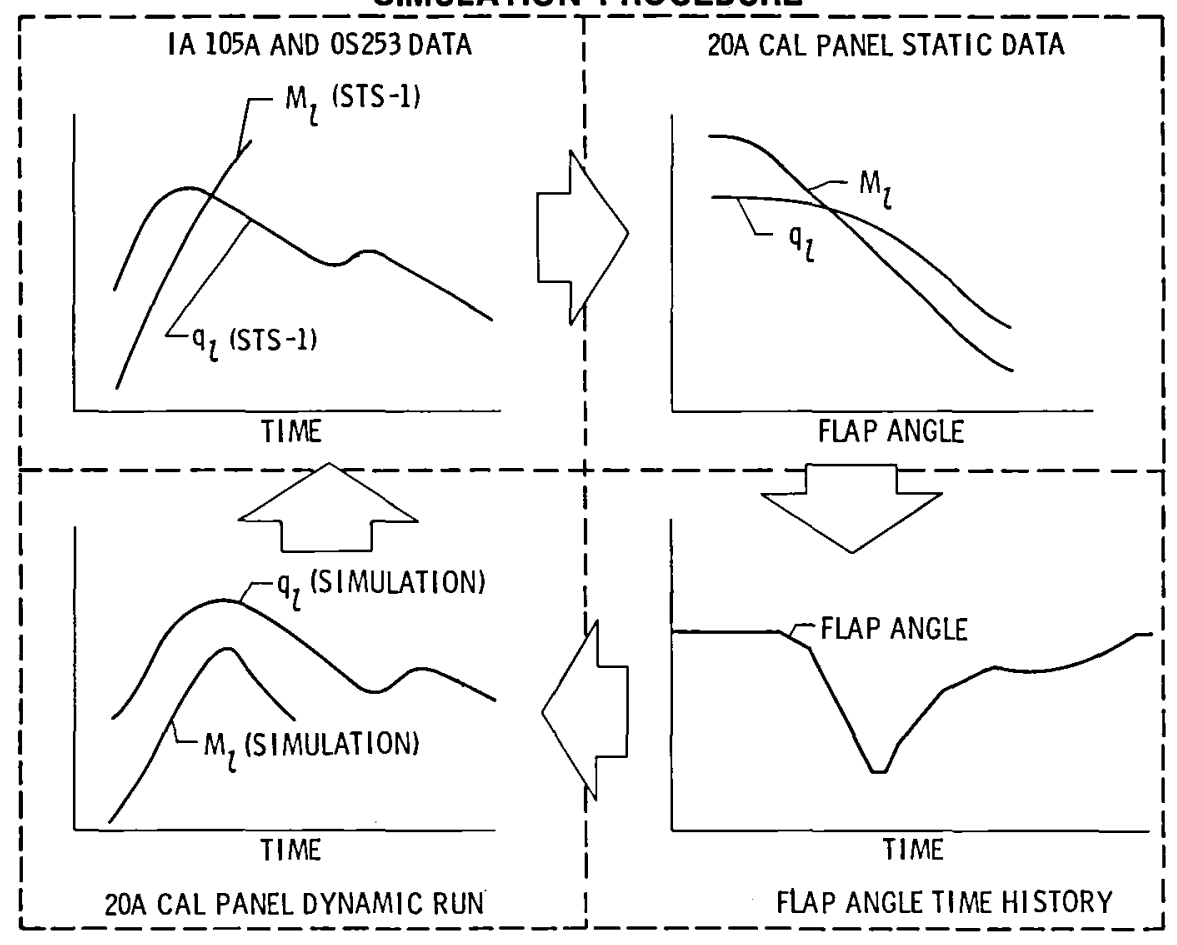

Figure 32.- Schematic illustrating steps required to simulate STS-1 time variations of local dynamic pressure and Mach number for panel $20 \mathrm{~A}$.

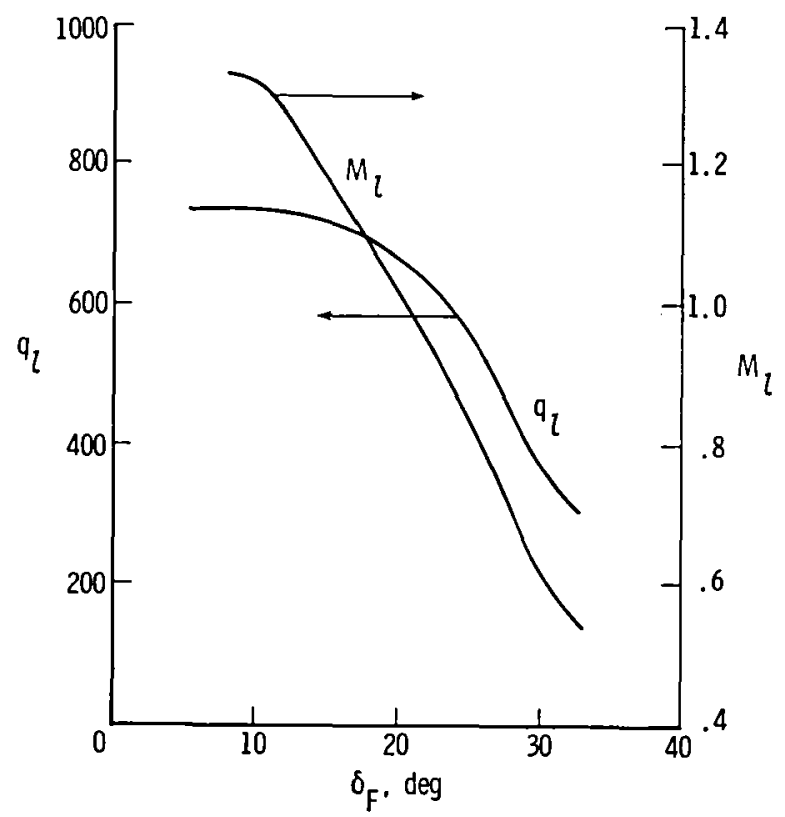

Figure 33.- Variation of local Mach number and dynamic pressure with flap angle for panel $20 \mathrm{~A}$. 


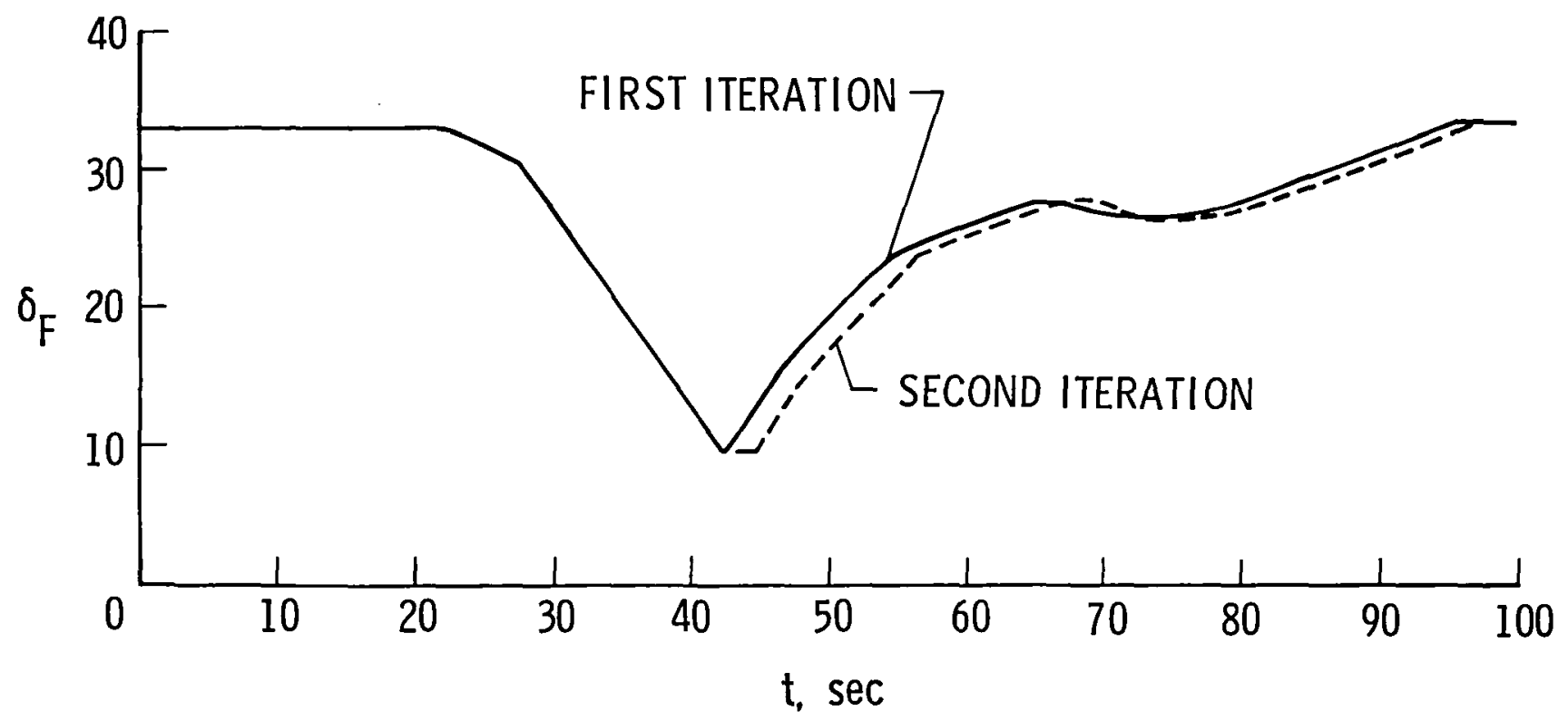

Figure 34.- Variation of diffuser-flap angle with time for first and second iterations.

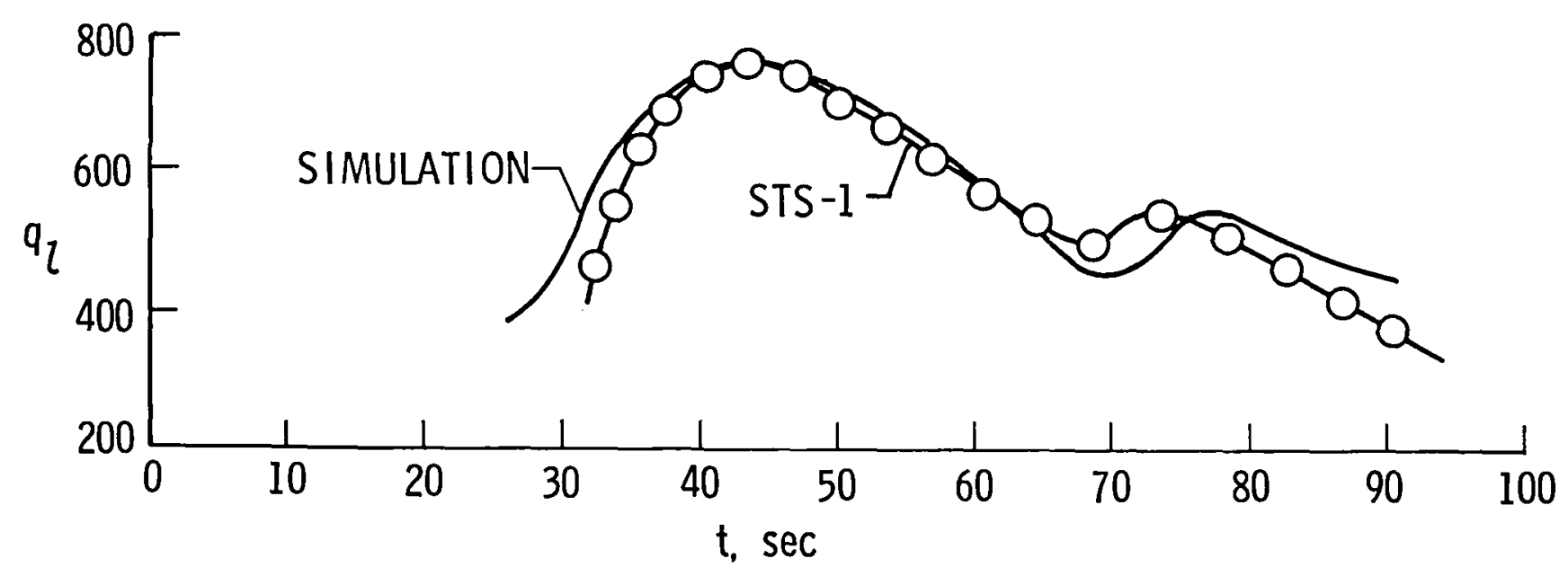

Figure 35.- Comparison of local dynamic-pressure time history for STS-1 with that obtained in 8-ft TPT simulation. 


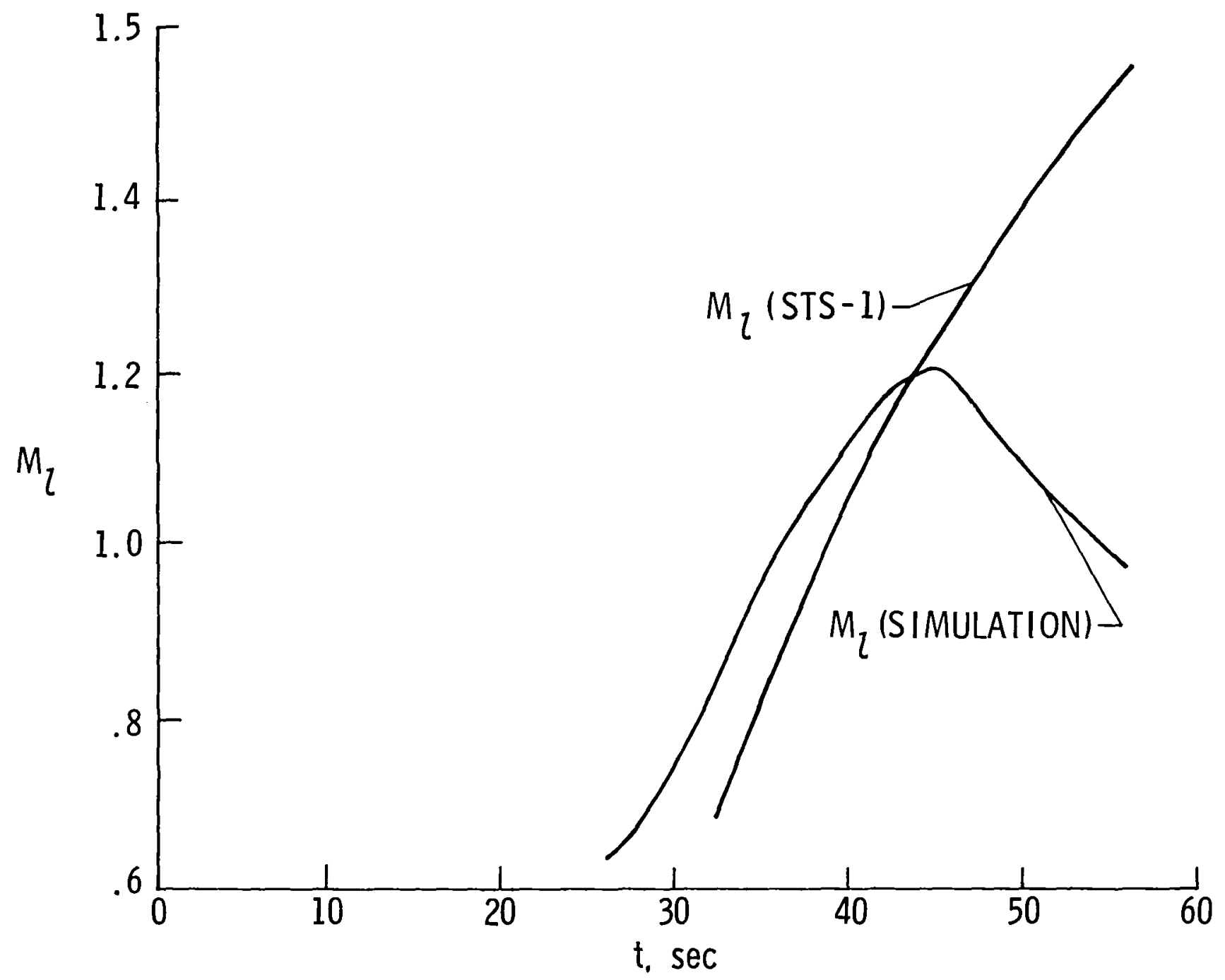

Figure 36.- Comparison of local Mach number time history for STS-1 with that obtained in 8-ft TPT simulation. 


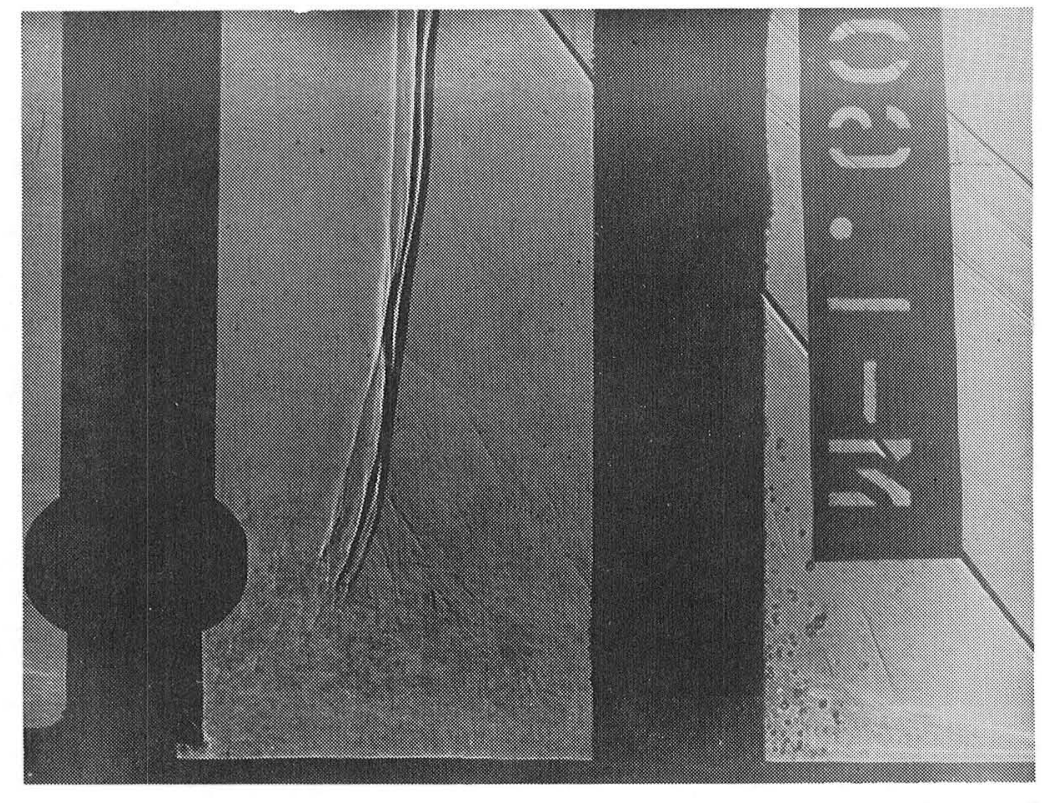

$L-82-185$

(a) Langley Unitary Plan Wind Tunnel.

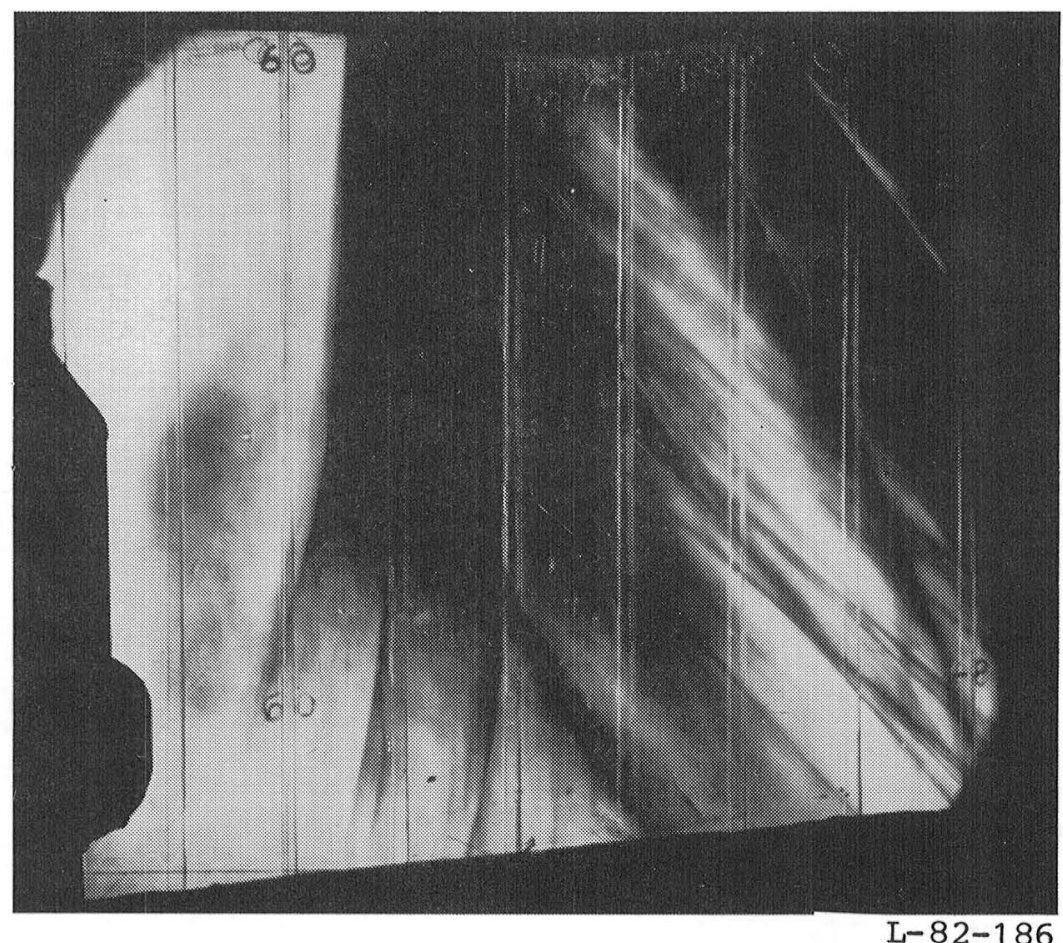

(b) Langley 8-Foot Transonic Pressure Tunnel.

Figure 37.- Shadowgraph and schlieren photographs from UPWT and 8-ft TPT at a Mach number of approximately 1.6 . 
NOTE: ONE MISSION DEFINED

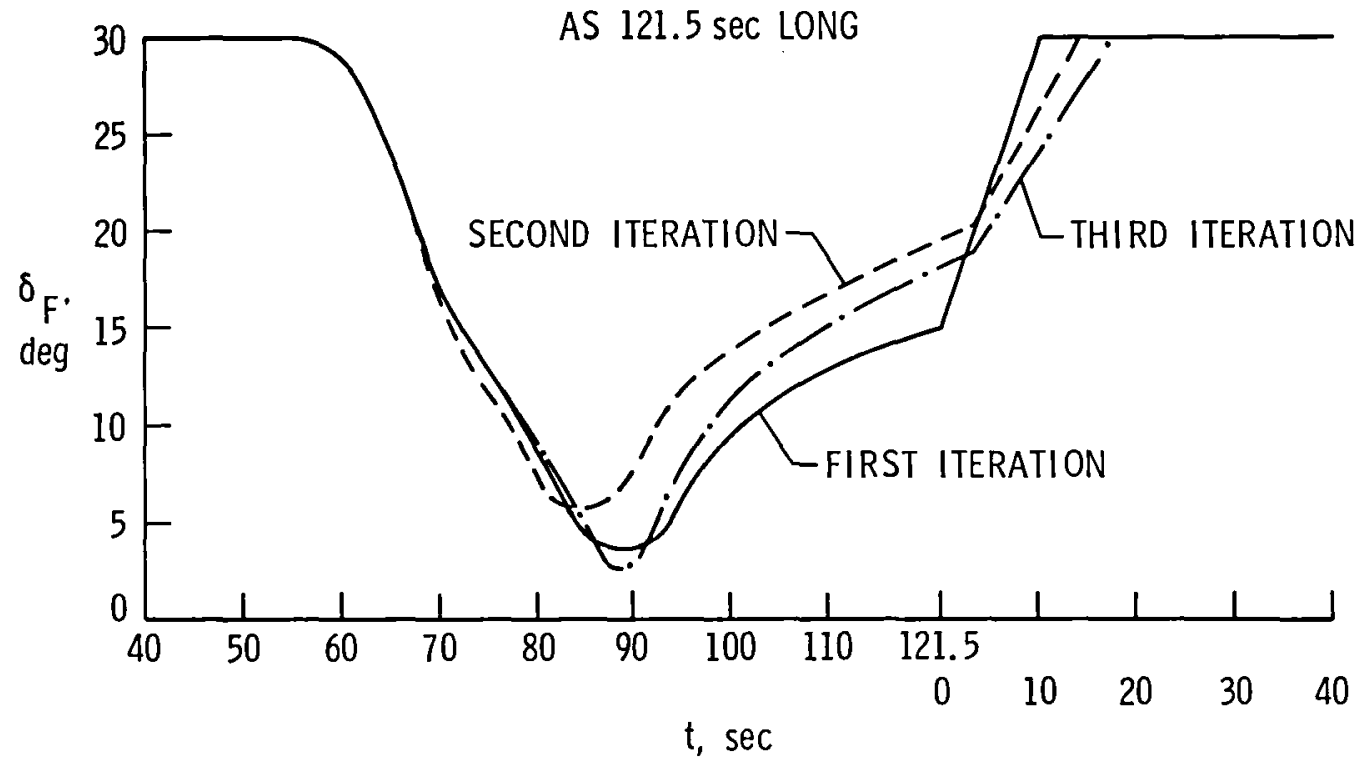

Figure 38.- Time variations of diffuser-flap angle for the three iterations attempted.

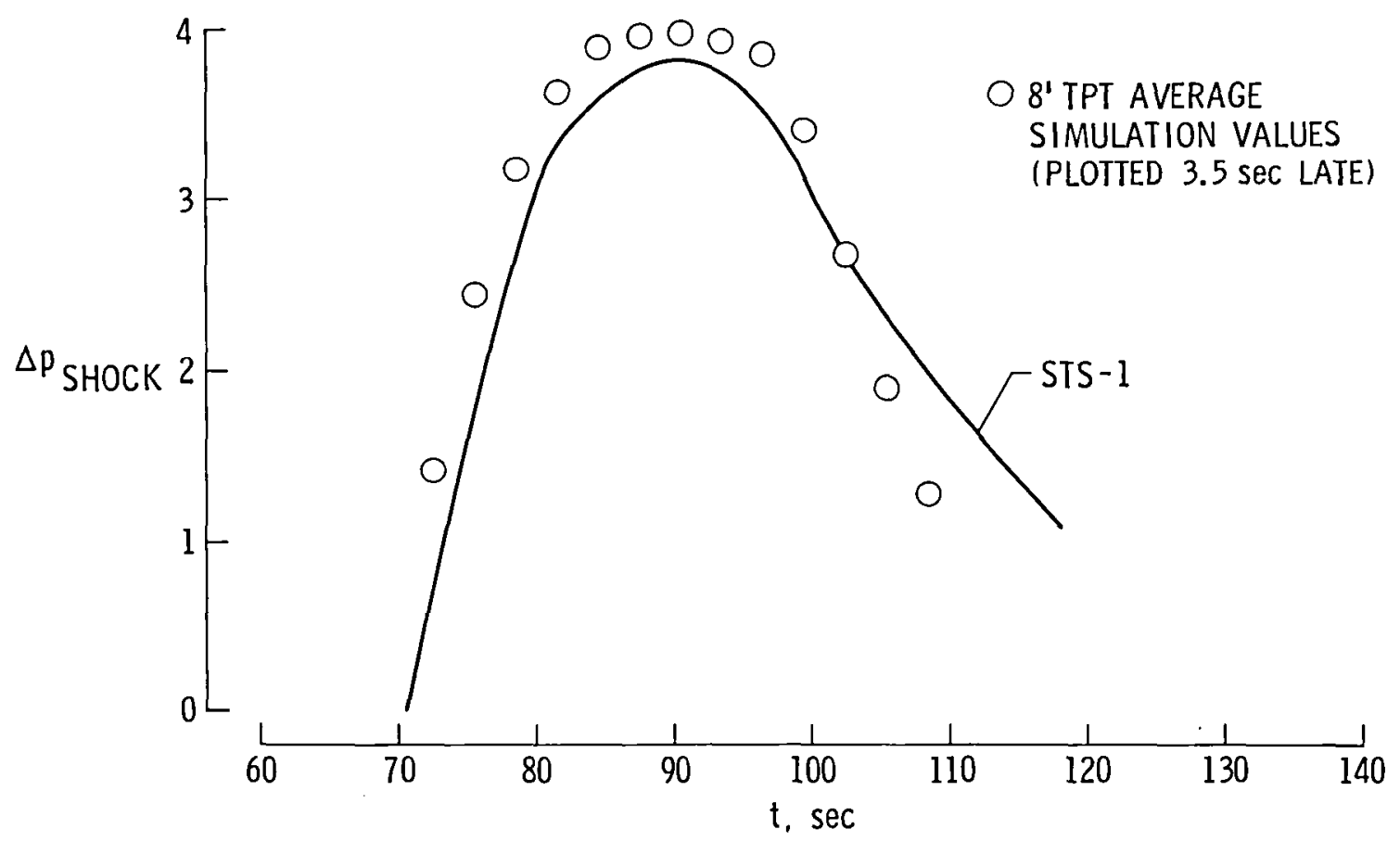

Figure 39.- Variations of bow-shock pressure jump $\Delta \mathrm{p}_{\mathrm{SHOCK}}$ with time for STS-1 and for 8-ft TPT simulation. 


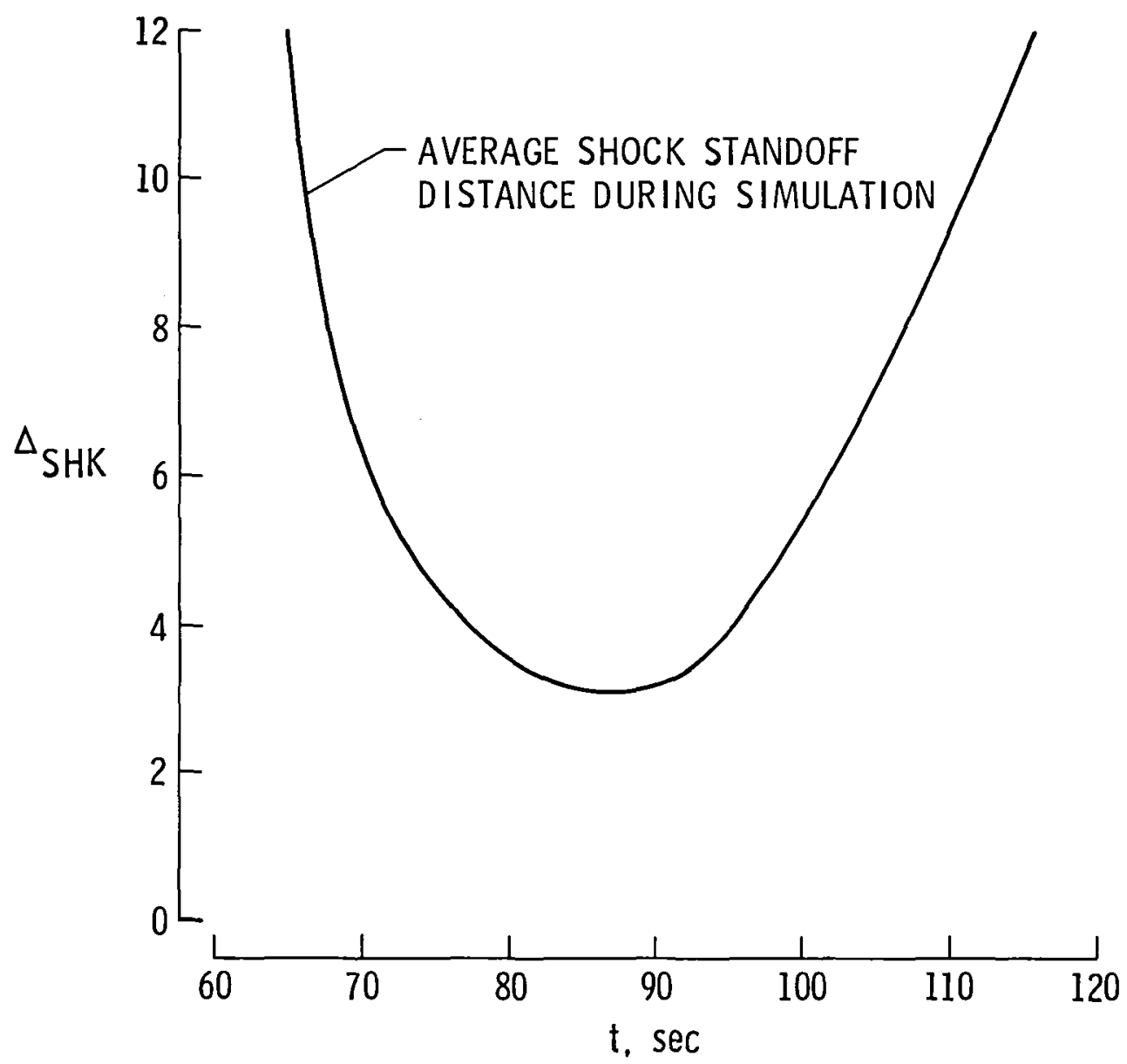

Figure 40.- Variation of average shock detachment distance with time based on six load cycles.

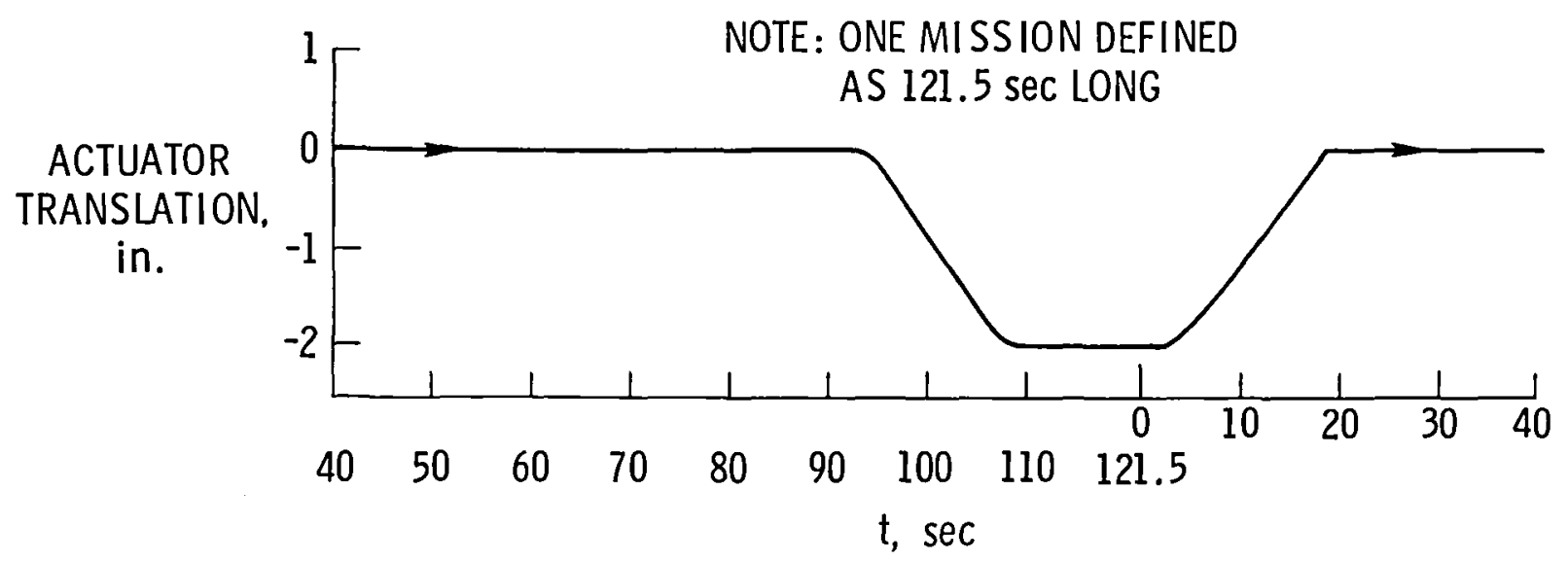

Figure 41.- Variation of position of half-scale bipod with time. 


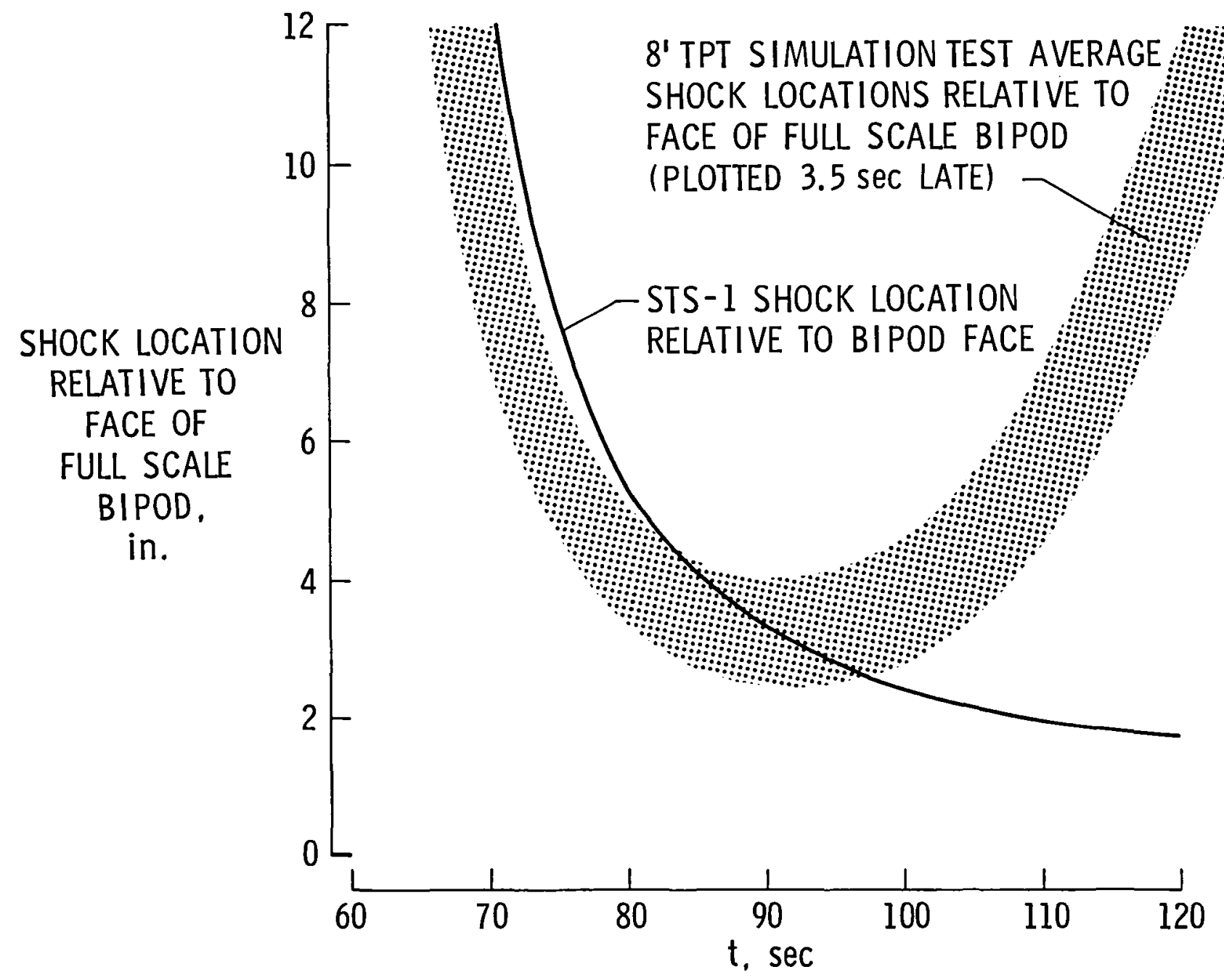

Figure 42.- Comparison of time variation of location of STS-1 bow shock relative to face of bipod with location of shock in 8-ft TPT simulation. 


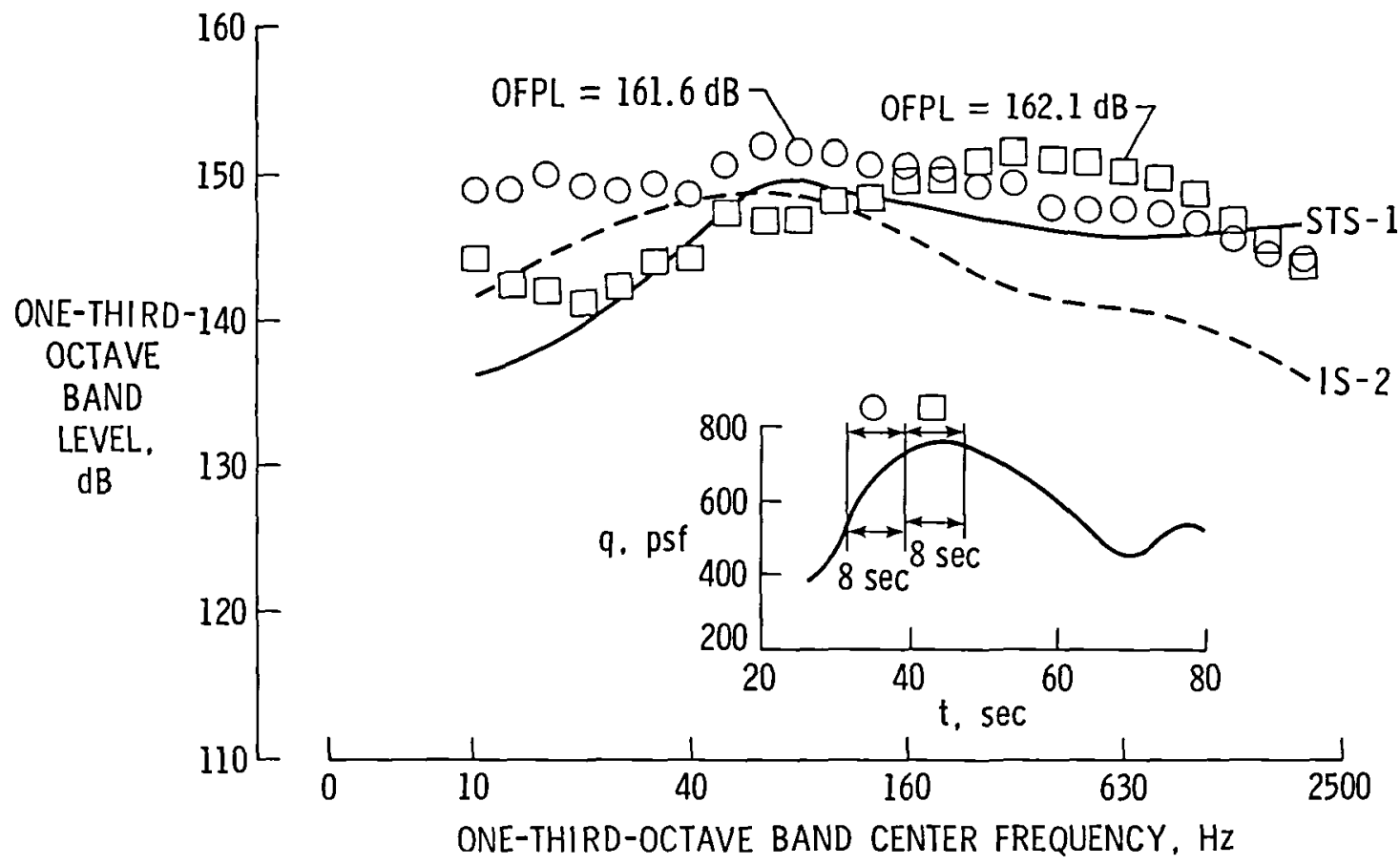

(a) Measurement made at a point $21.4 \mathrm{in}$. ahead of panel trailing edge.

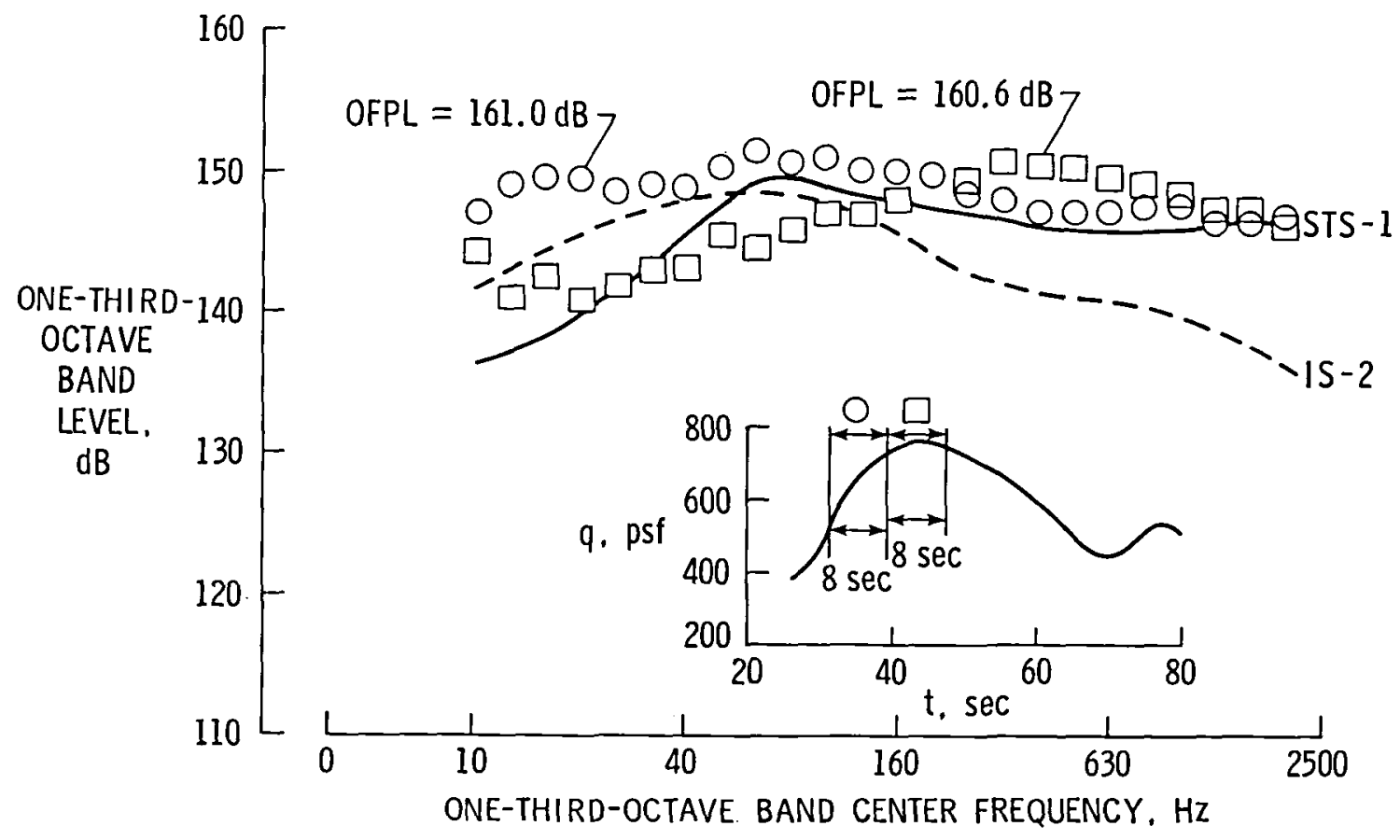

(b) Measurement made at a point 10.5 in. ahead of trailing edge.

Figure 43.- Representative variations of one-third-octave band sound pressure level with one-third-octave band center frequencies for two locations and for two separate time intervals on panel 20A. Measurements made for an IS-2 test and STS-1 flight. 


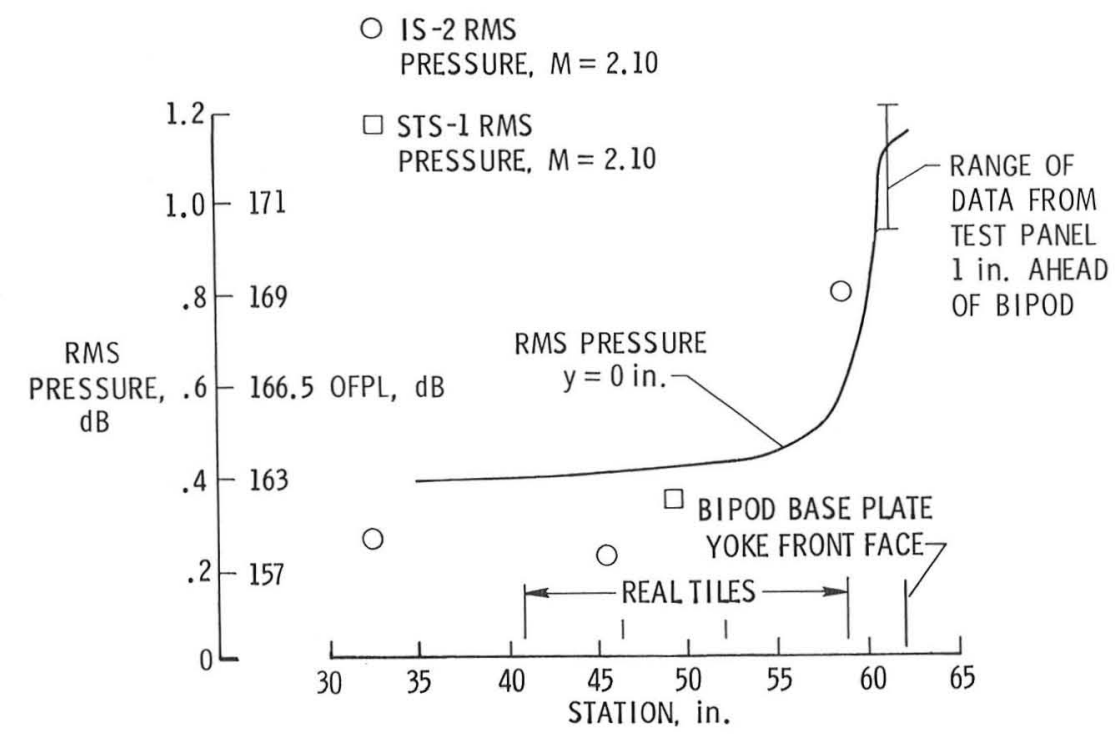

Figure 44.- Variation of rms sound pressures ahead of bipod from panel $20 \mathrm{C}$ tests compared with IS-2 and STS-1 results.

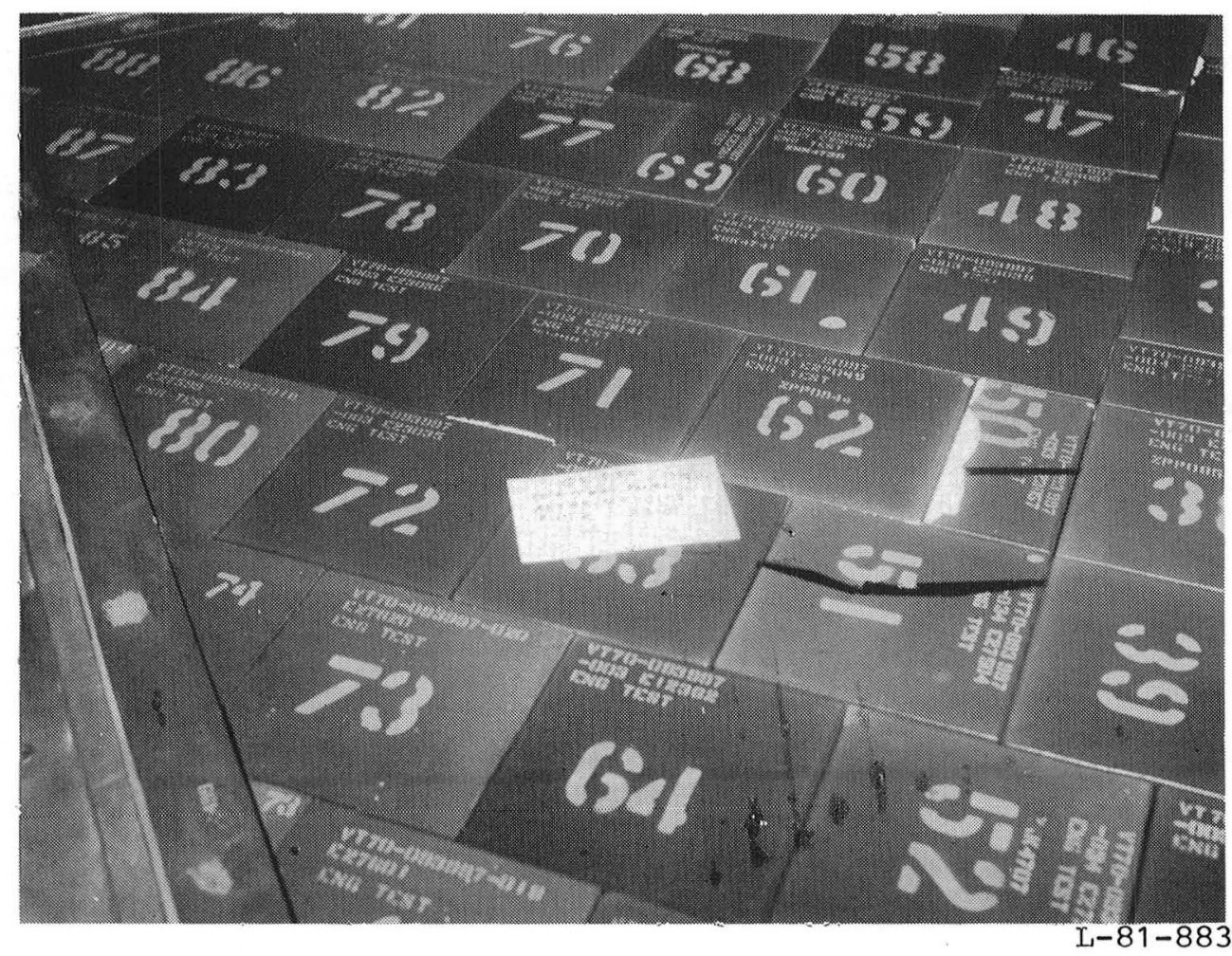

Figure 45.- Photograph showing chipped coating of tiles on panel 20A after 76 load cycles. 



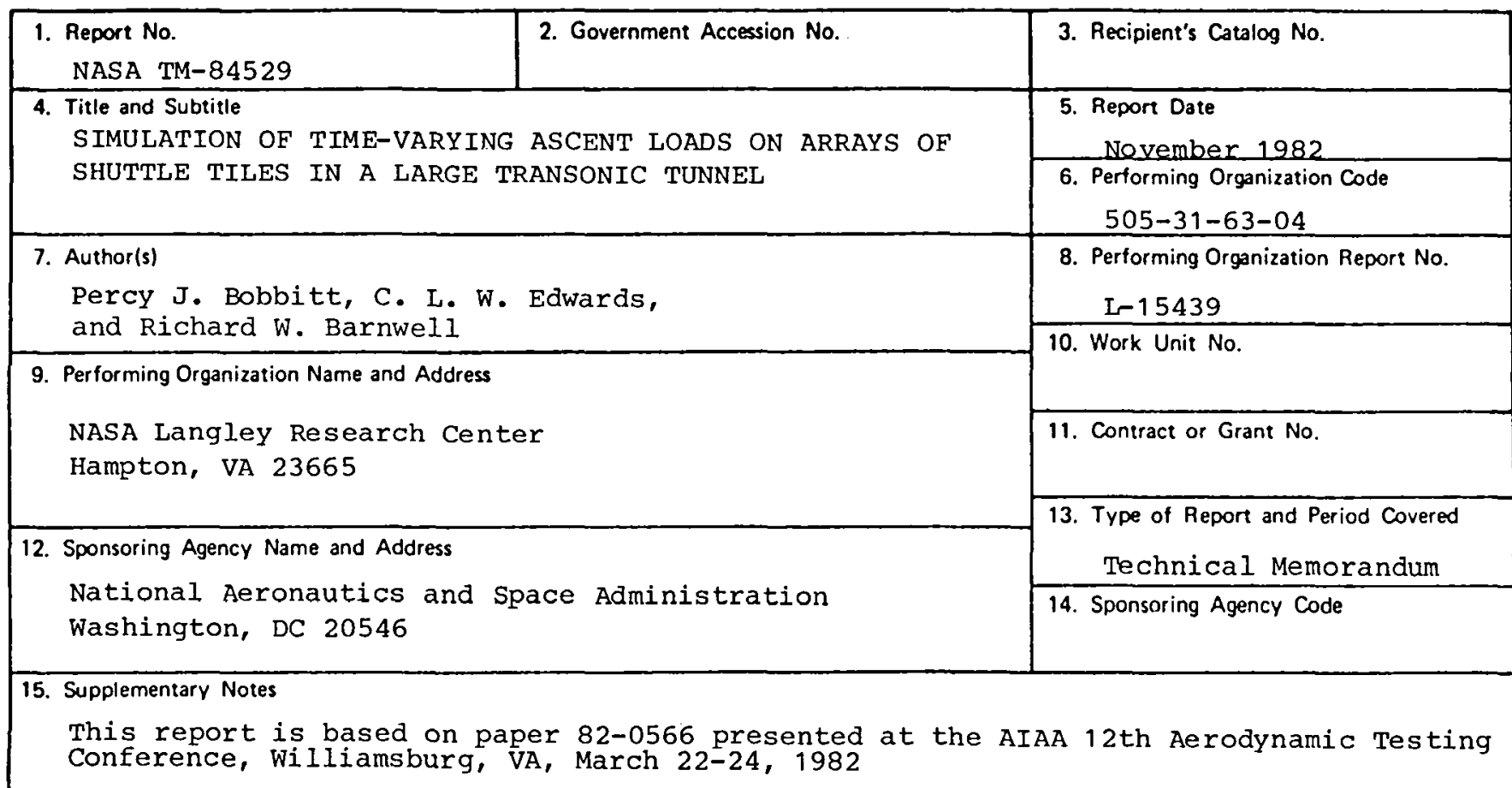

16. Abstract

This paper describes first-flight-critical tests conducted to simulate the time histories of Shuttle ascent loads on actual tiles bonded to accurate representations of orbiter flight structures. Diffuser spoiler flaps were used to generate appropriate variations of free-stream conditions in real time in the Langley 8-Foot Transonic Pressure Tunnel. Time histories of the critical-load parameters imposed on the tile arrays in the tunnel are compared with those expected in flight.

17. Key Words (Suggested by Author(s))

Unsteady aerodynamic loads

Flight structures

shuttle tiles
18. Distribution Statement

Unclassified - Un limited

Subject Category 16

19. Security Classif. (of this report)

20. Security Classif. (of this page)

Unclassified Unclassified

21. No. of Pages

50

$\mathrm{A} 03$

For sale by the National Technical Information Service, Springfield, Virginia 22161 

National Aeronautics and Space Administration

Washington, D.C.

20546

\section{Official Business}

Penalty for Private Use, $\$ 300$
Postage and Fees Paid National Aeronautics and Space Administration NASA-451 